\title{
Synthesis of Isoquinolones by Sequential Suzuki Coupling of 2-Halobenzonitriles with Vinyl Boronate Followed by
}

Cyclization

Saul Jaime-Figueroa ${ }^{1}$, Michael J. Bond ${ }^{2}$, J. Ignacio Vergara ${ }^{3}$, Jake C. Swartzel ${ }^{3}$, Craig M. Crews ${ }^{1,2,3 *}$.

${ }^{1}$ Department of Molecular, Cellular \& Developmental Biology, Yale University, New Haven, CT 06511, USA.

2Department of Pharmacology, Yale University, New Haven, CT 06511, USA

${ }^{3}$ Department of Chemistry, Yale University, New Haven, CT 06511, USA.

\section{Table of Contents}

S1. NMR spectra for compounds $2 \mathrm{a}-20$

S2. NMR spectra for compounds 3a-3o.

S3. NMR spectra for compounds $6 a-6 \mathrm{~d}$

S4. NMR spectra for compounds 7a-7d

S5. NMR spectra for compounds 10 and 7d'. 
S1. NMR spectra for compounds 2a-2o.

Figure S1. ${ }^{1} \mathrm{H}-\mathrm{NMR}$ (400 MHz, DMSO-d6) for compound 2a.

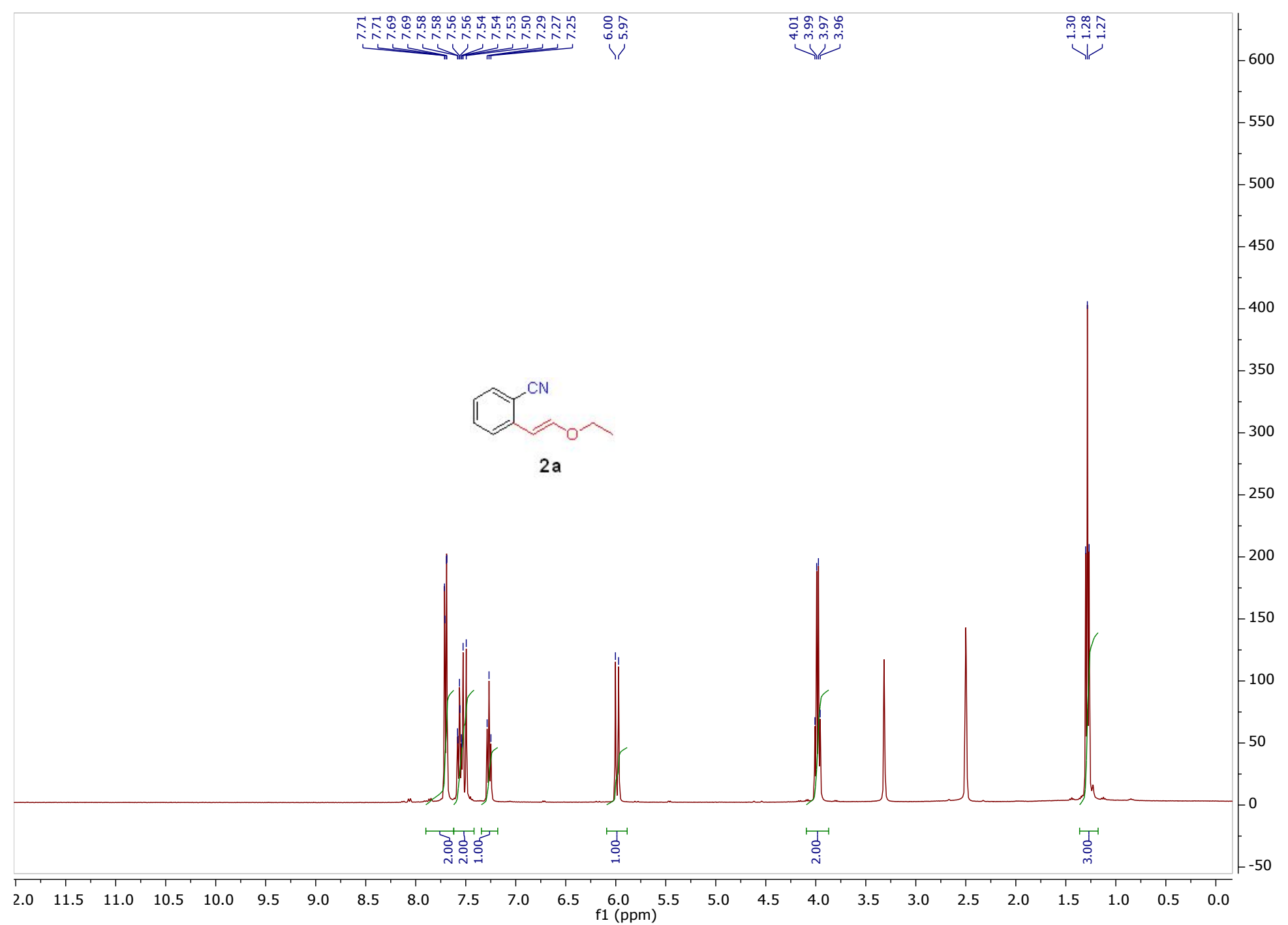


Figure S2. ${ }^{13} \mathrm{C}\left\{{ }^{1} \mathrm{H}\right\}--\mathrm{NMR}$ (101 MHz, DMSO-d6) for compound 2a.

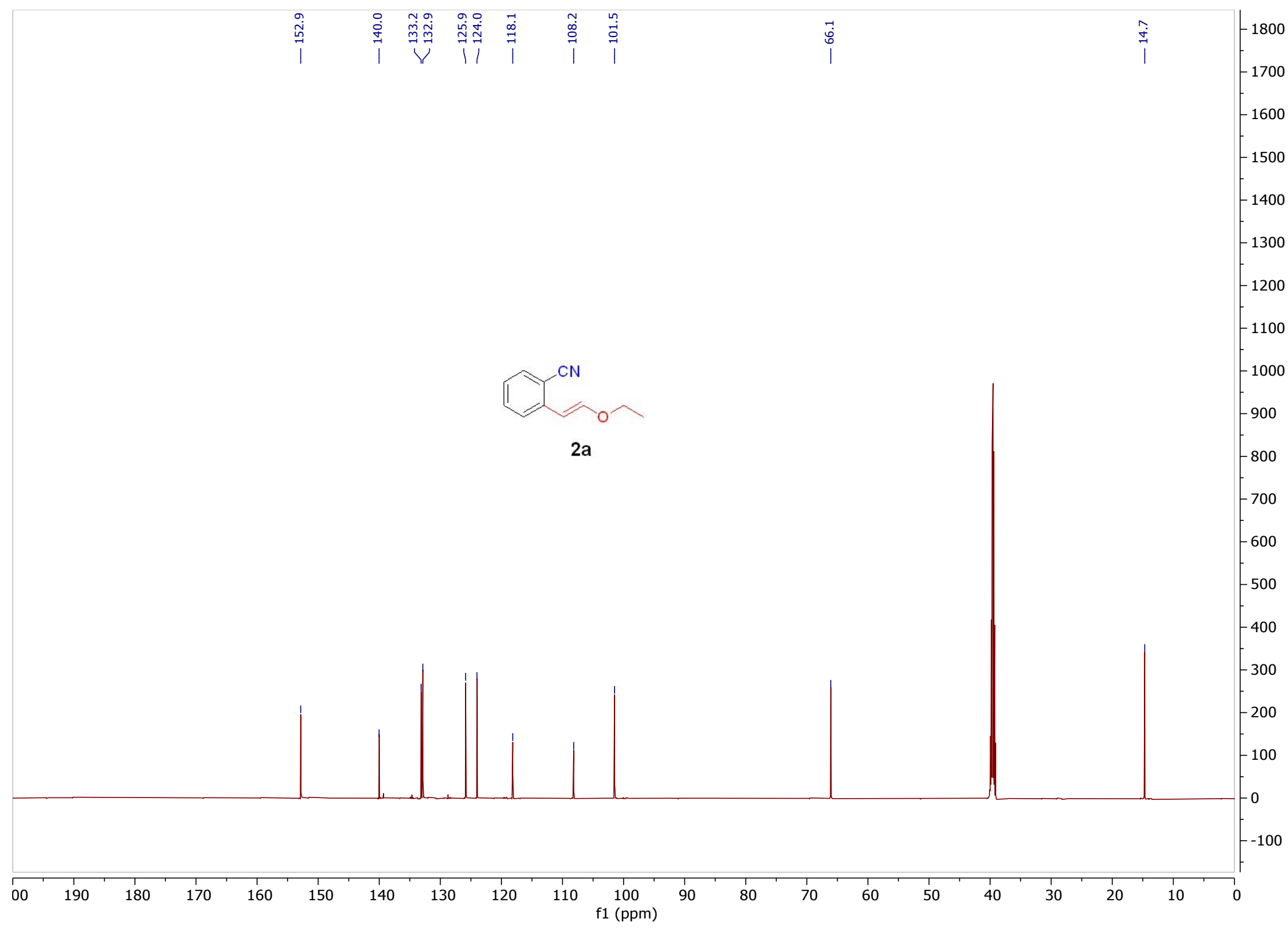


Figure S3. ${ }^{13} \mathrm{H}-\mathrm{NMR}\left(400 \mathrm{MHz}, \mathrm{CDCl}_{3}\right)$ for compound $2 \mathrm{~b}$.

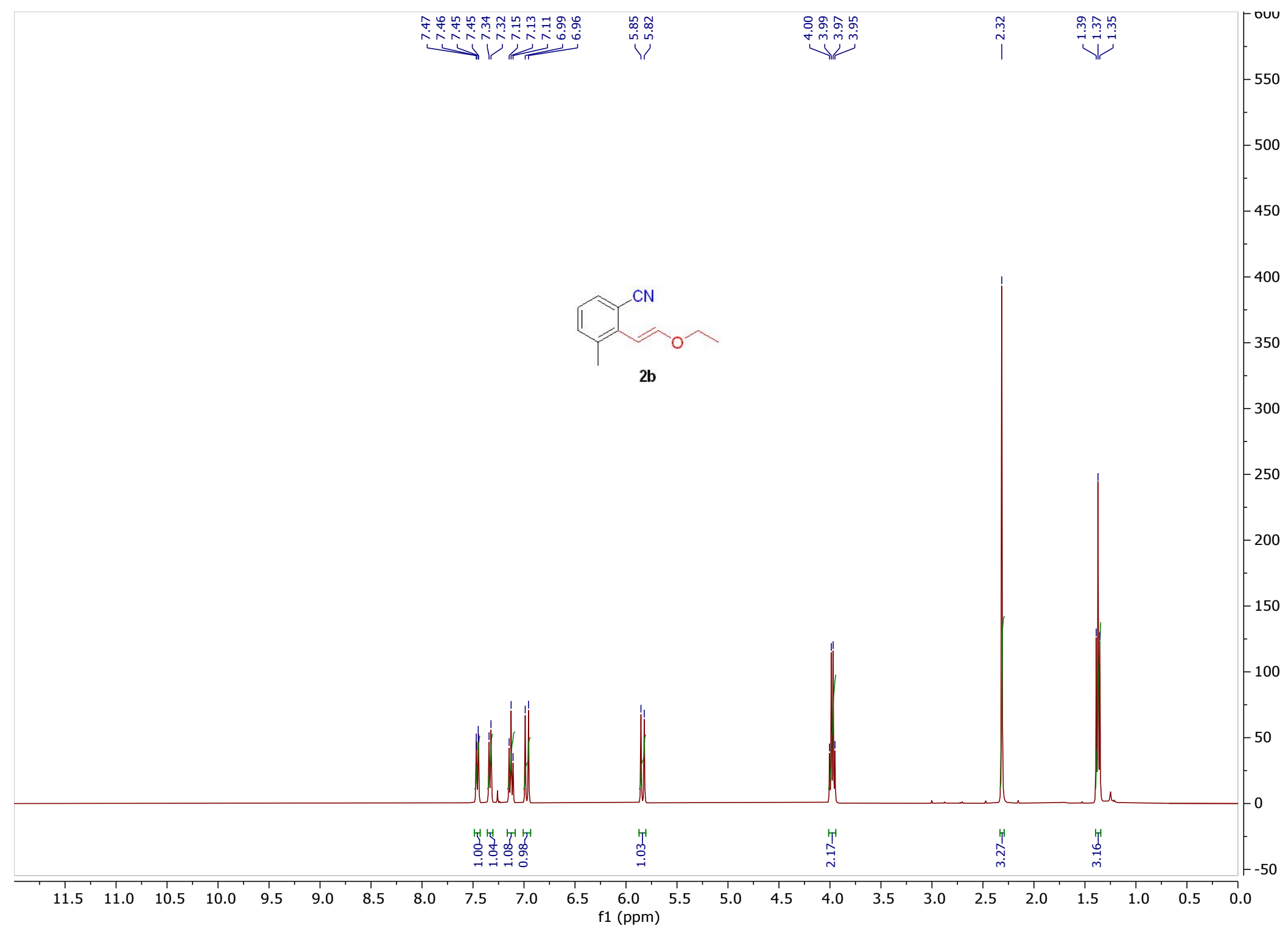


Figure S4. ${ }^{13} \mathrm{C}\left\{{ }^{1} \mathrm{H}\right\}-\mathrm{NMR}\left(101 \mathrm{MHz}, \mathrm{CDCl}_{3}\right)$ for compound $2 \mathrm{~b}$.

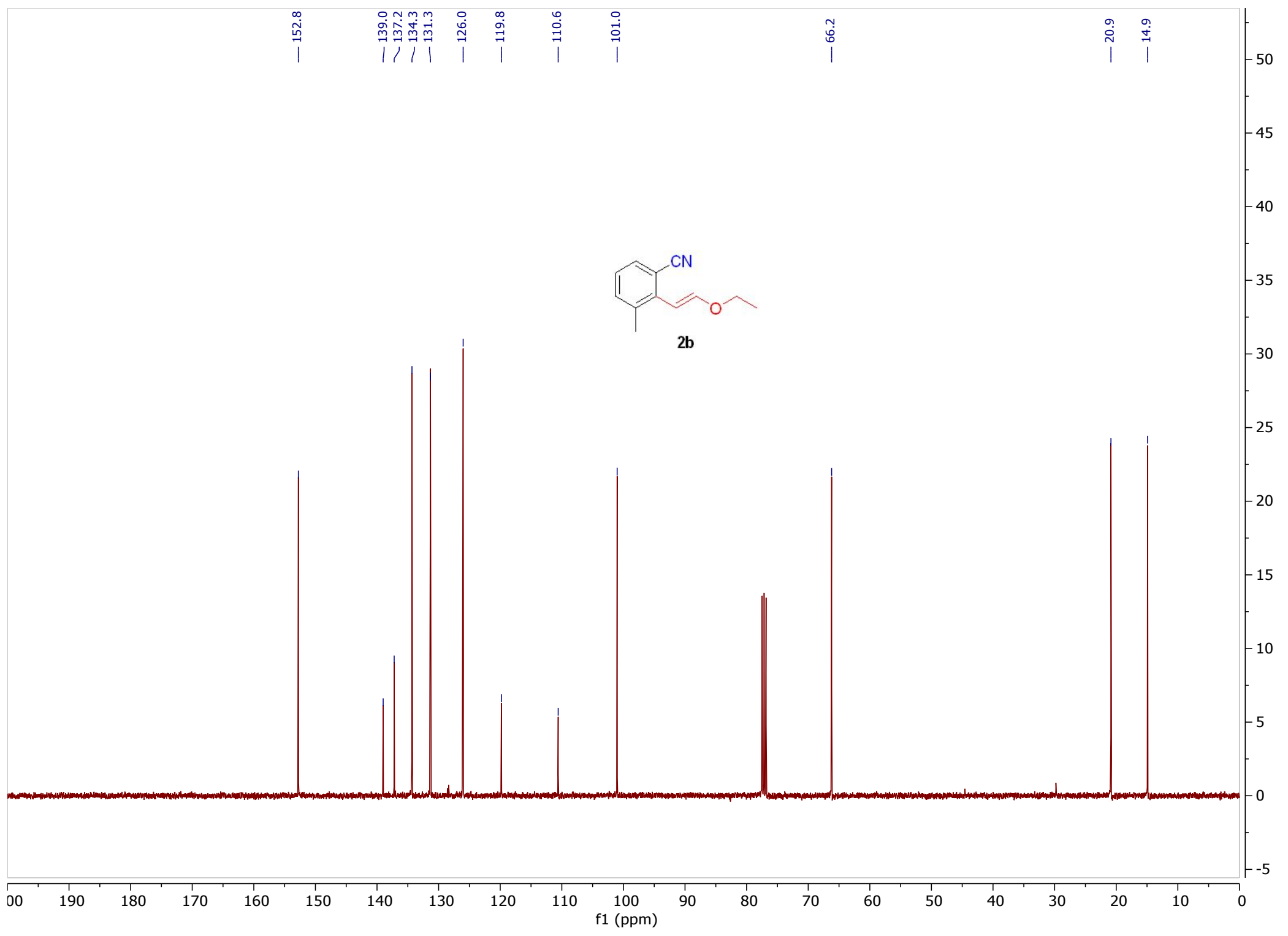

SI-5 
Figure $\mathrm{S} 5 .{ }^{1} \mathrm{H}-\mathrm{NMR}\left(400 \mathrm{MHz}, \mathrm{CDCl}_{3}\right)$ for compound $2 \mathrm{c}$.

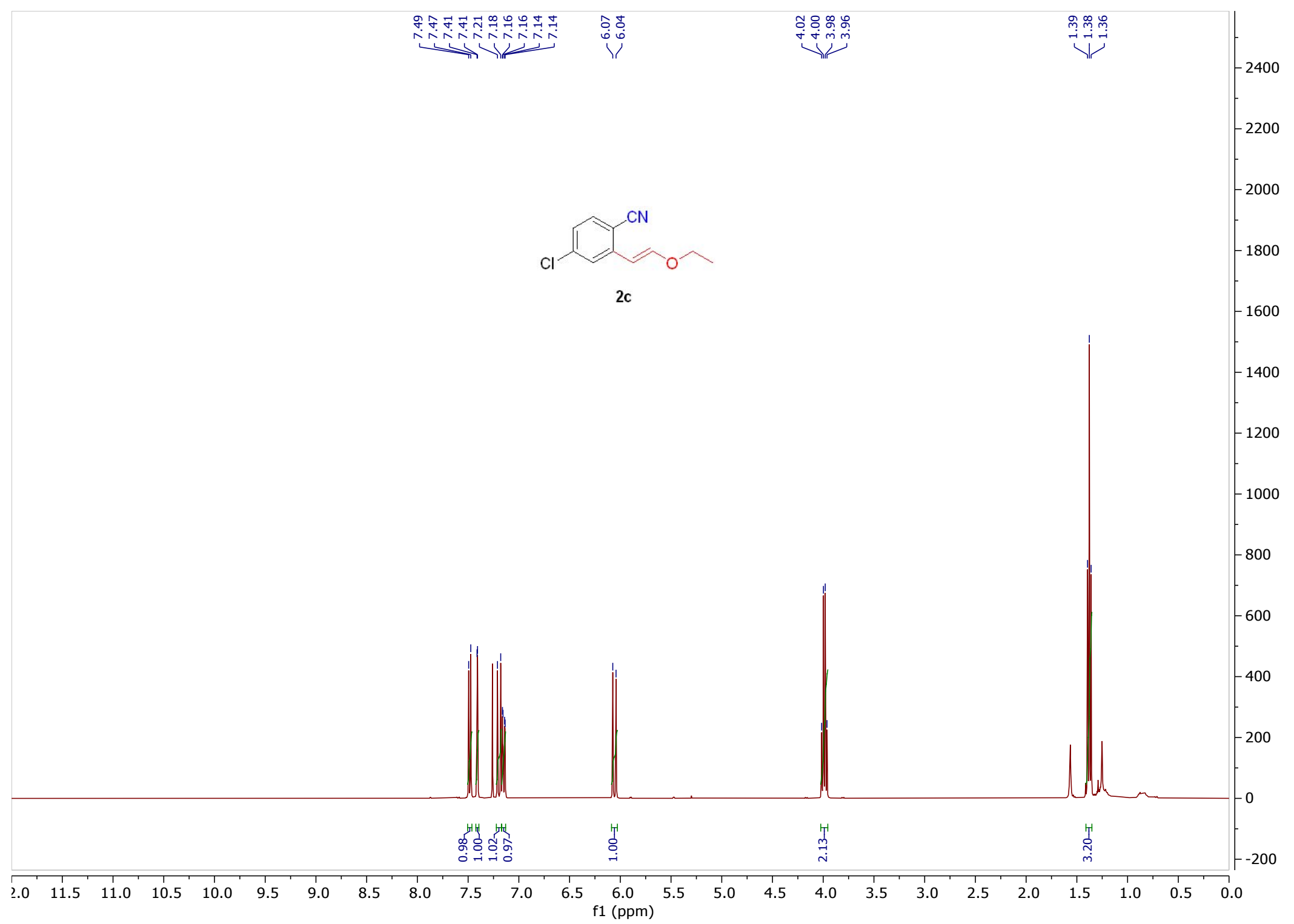


Figure S6. ${ }^{13} \mathrm{C}\left\{{ }^{1} \mathrm{H}\right\}-\mathrm{NMR}\left(101 \mathrm{MHz}, \mathrm{CDCl}_{3}\right)$ for compound $2 \mathrm{c}$.

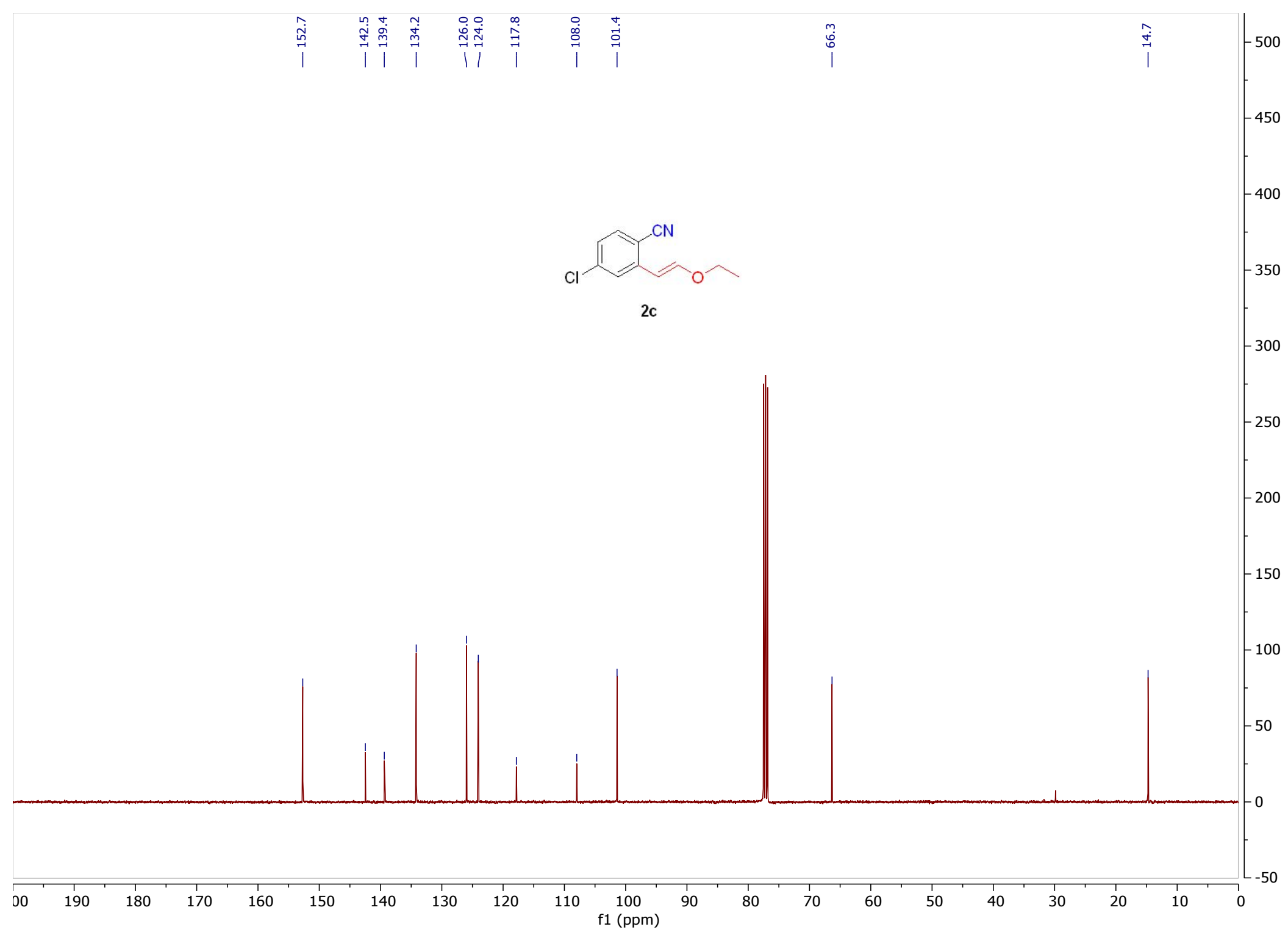


Figure S7. ${ }^{1} \mathrm{H}-\mathrm{NMR}$ (500 MHz, DMSO-d6) for compound $2 \mathrm{~d}$.

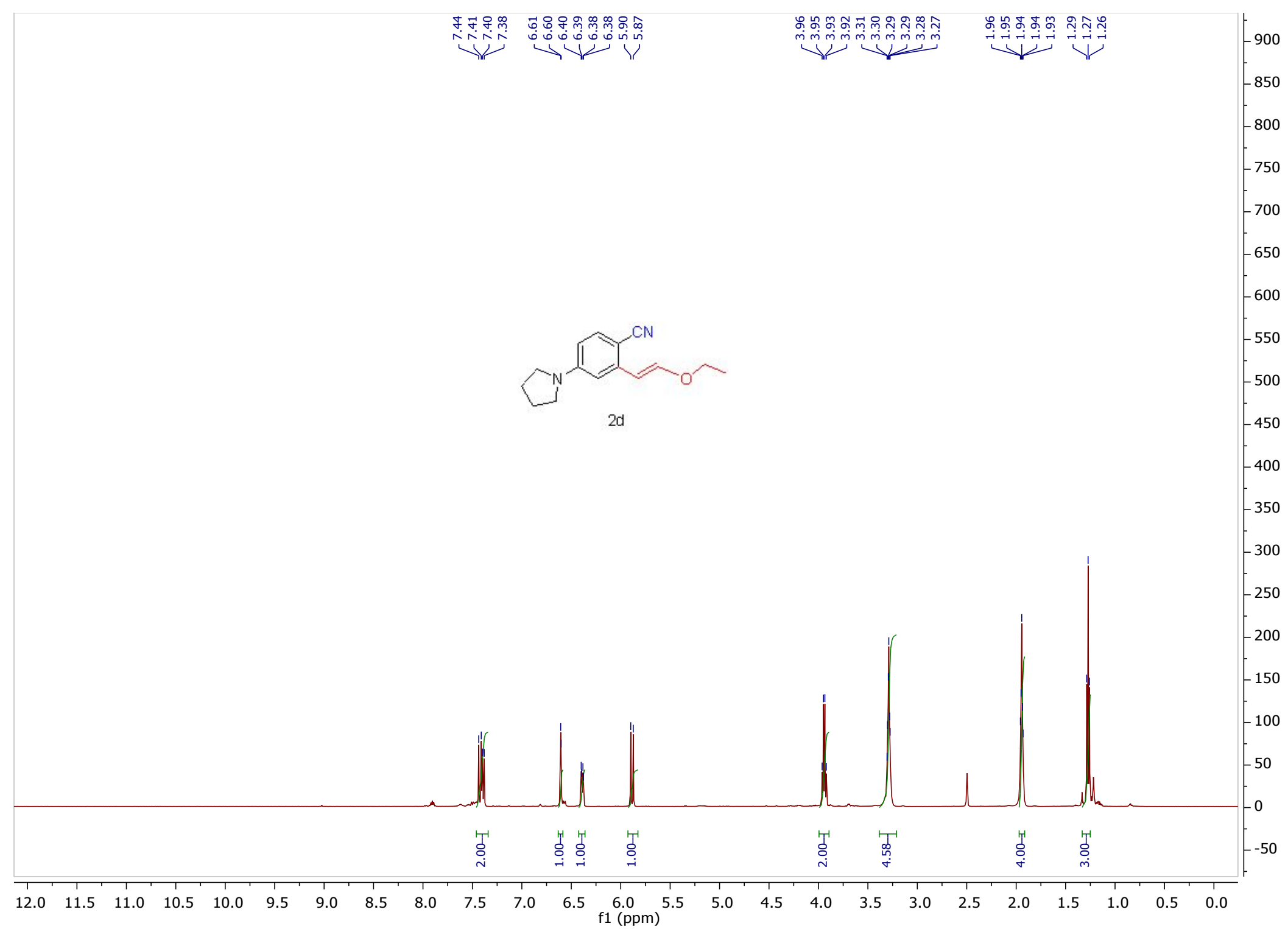


Figure S8. ${ }^{13} \mathrm{C}\left\{{ }^{1} \mathrm{H}\right\}-\mathrm{NMR}$ (126 MHz, DMSO-d6) for compound $2 \mathrm{~d}$.

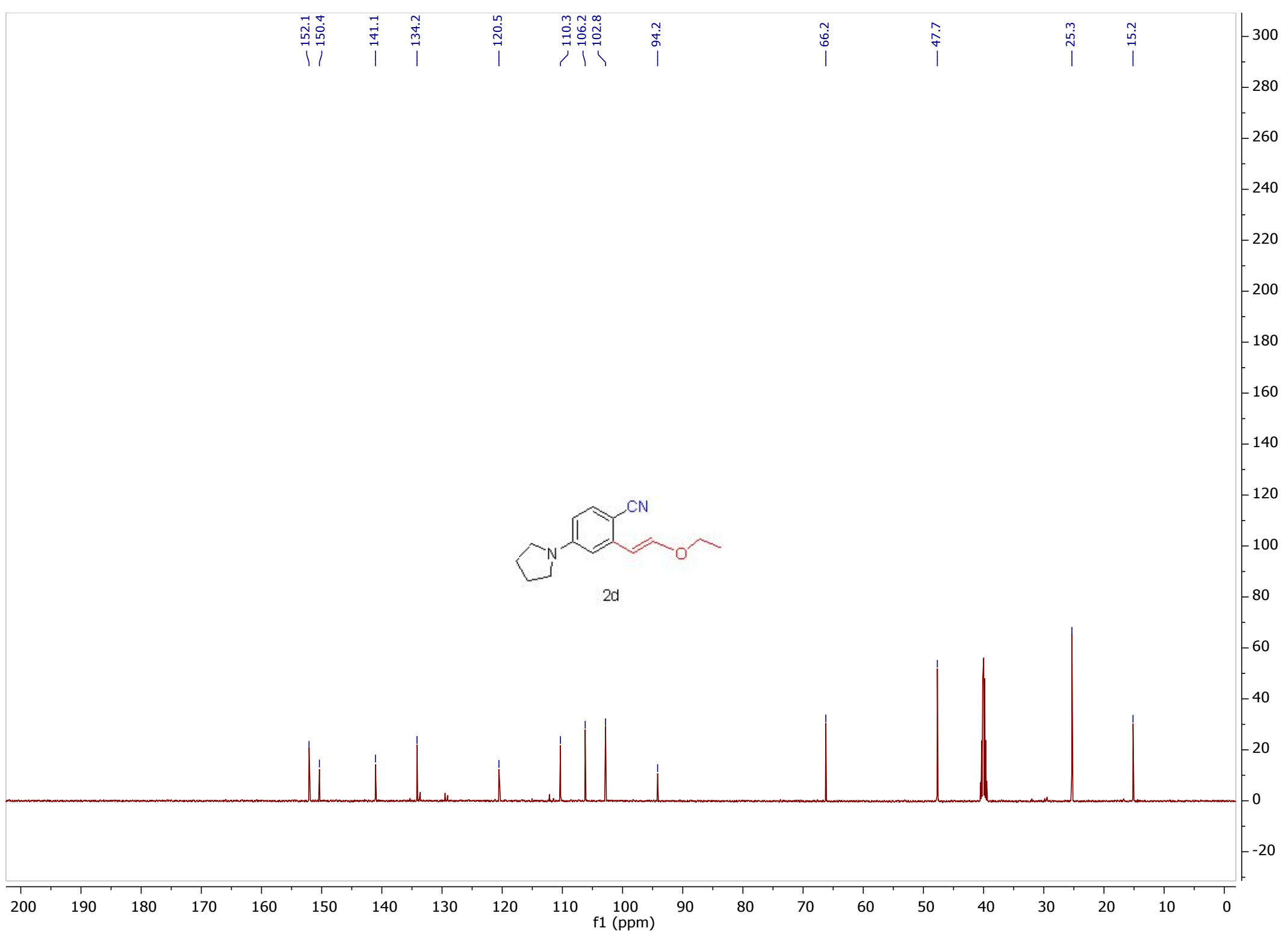


Figure S9. ${ }^{1} \mathrm{H}-\mathrm{NMR}\left(400 \mathrm{MHz}, \mathrm{CDCl}_{3}\right)$ for compound $2 \mathrm{e}$.

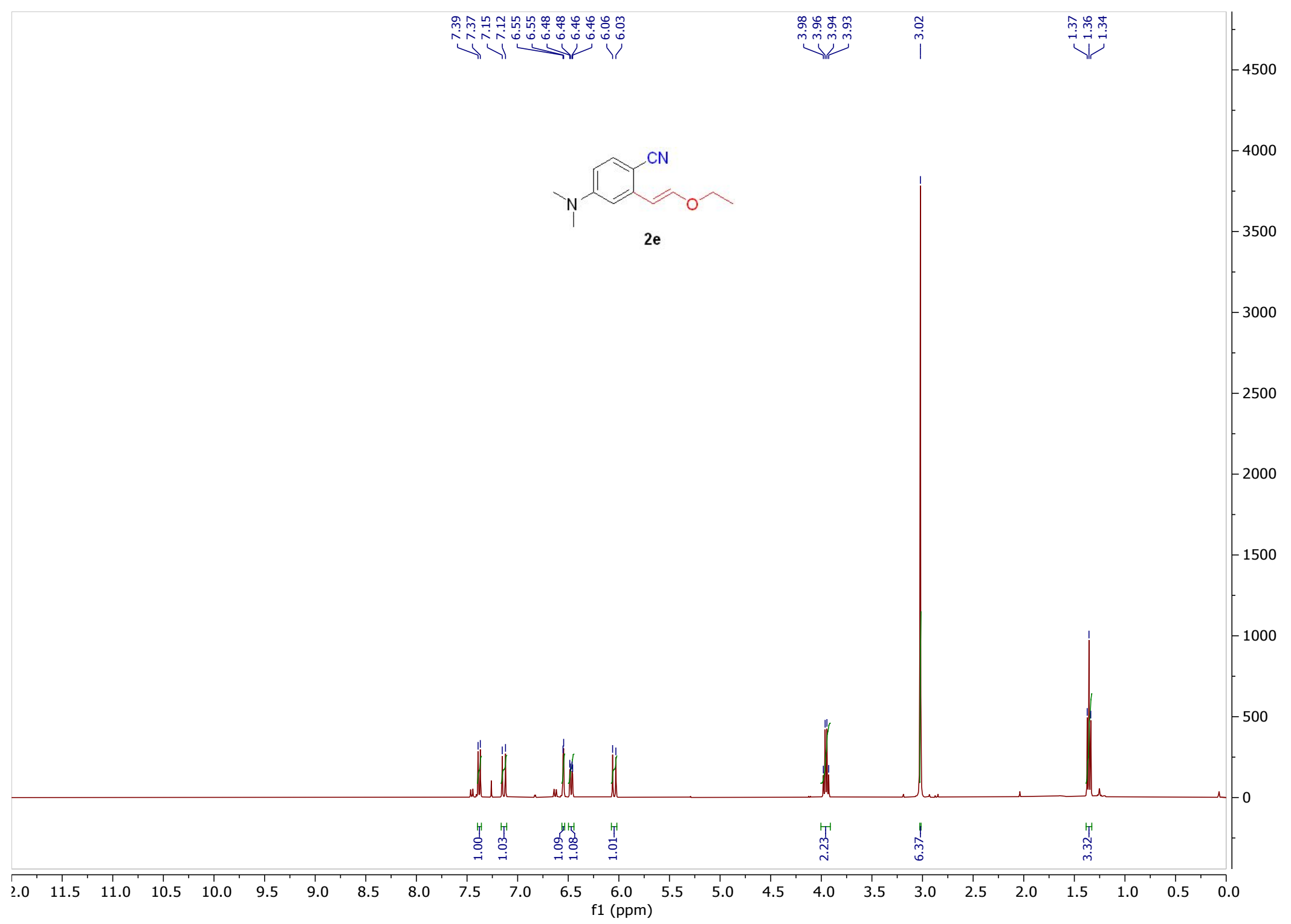


Figure S10. ${ }^{13} \mathrm{C}\left\{{ }^{1} \mathrm{H}\right\}-\mathrm{NMR}\left(101 \mathrm{MHz}, \mathrm{CDCl}_{3}\right)$ for compound $2 \mathrm{e}$.

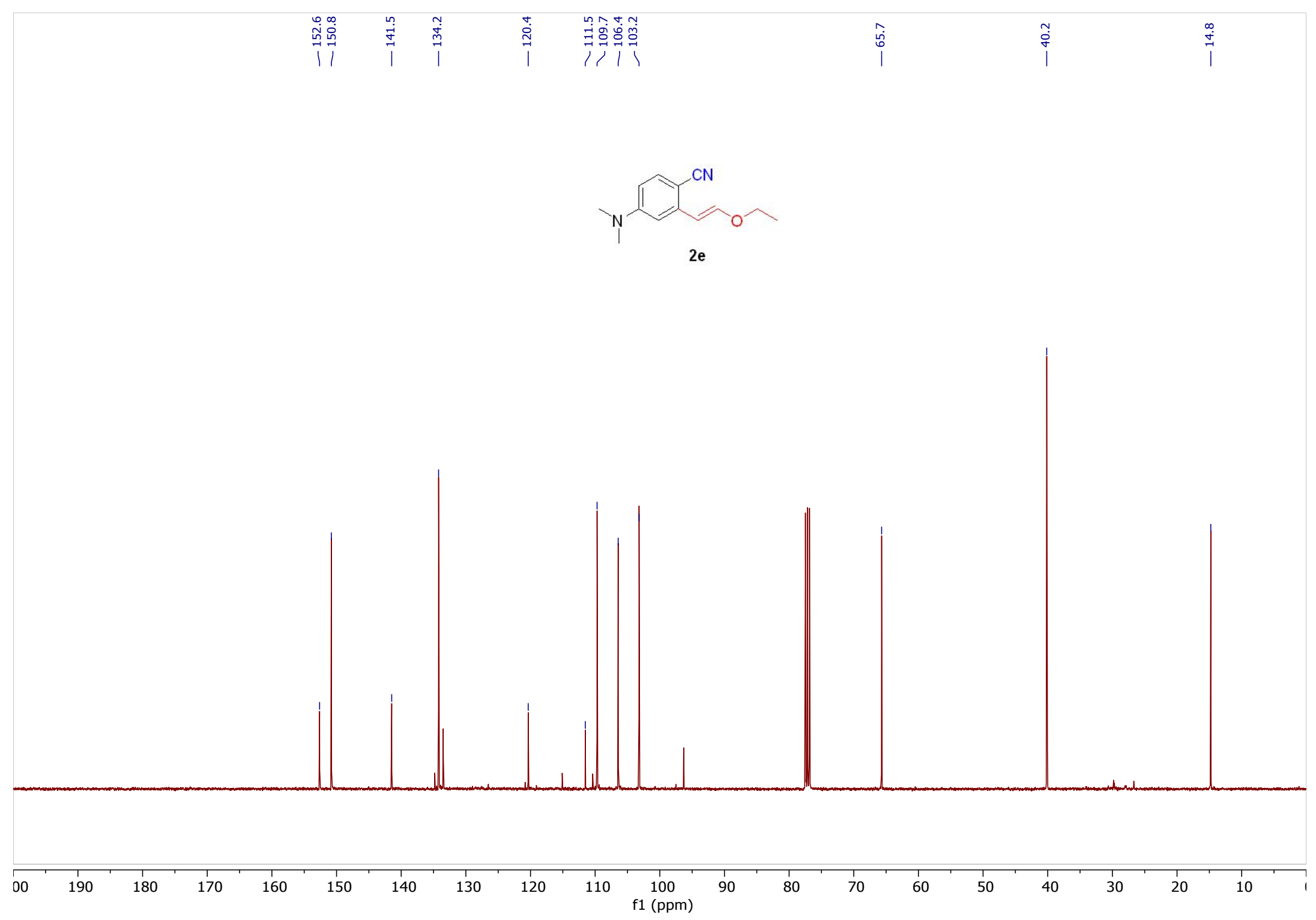


Figure S11. ${ }^{1} \mathrm{H}-\mathrm{NMR}$ (400 Hz, DMSO-d6) for compound $2 \mathrm{f}$.

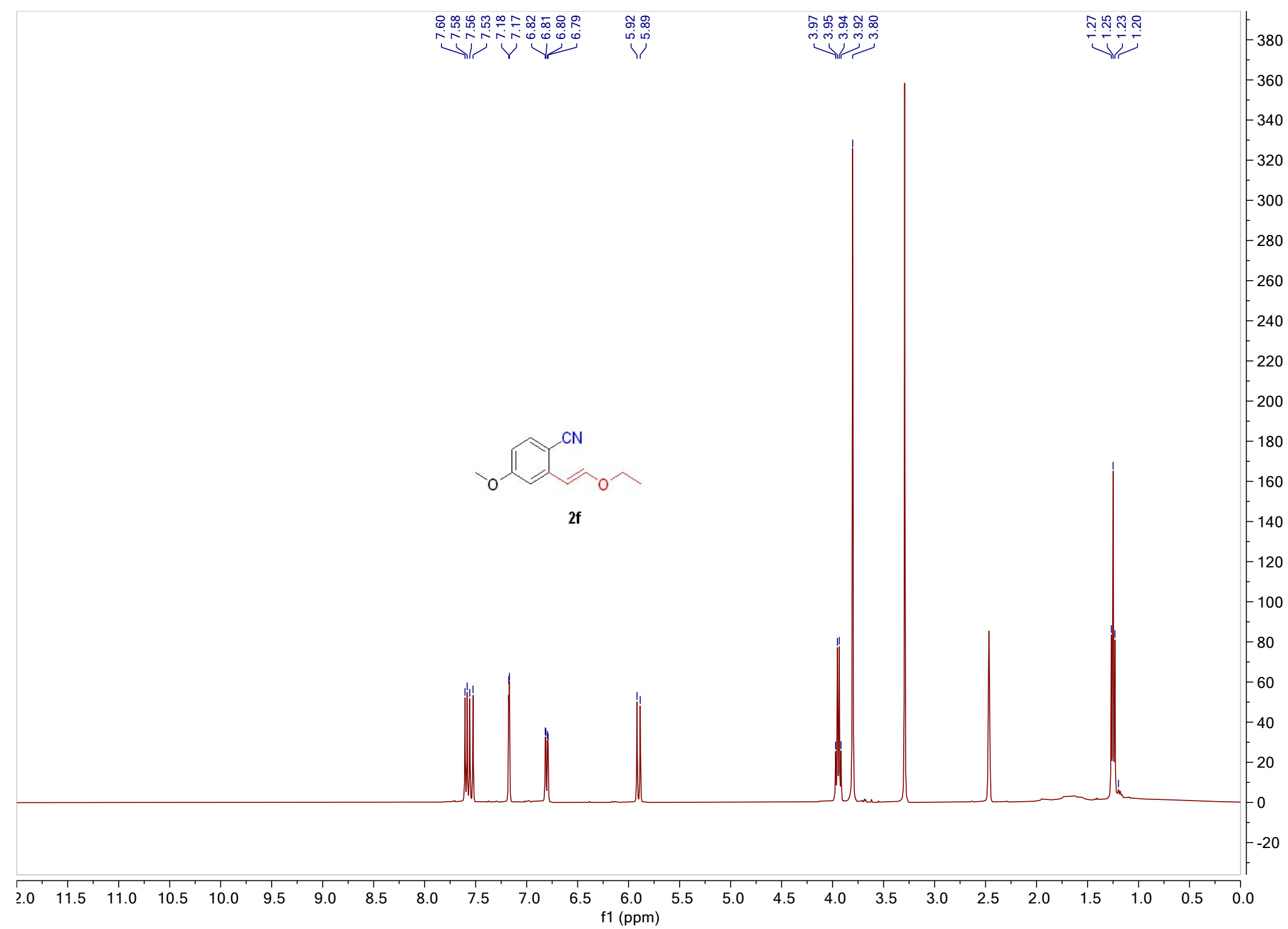


Figure S12. ${ }^{13} \mathrm{C}\left\{{ }^{1} \mathrm{H}\right\}-\mathrm{NMR}$ (101 MHz, DMSO-d6) for compound $2 \mathrm{f}$.

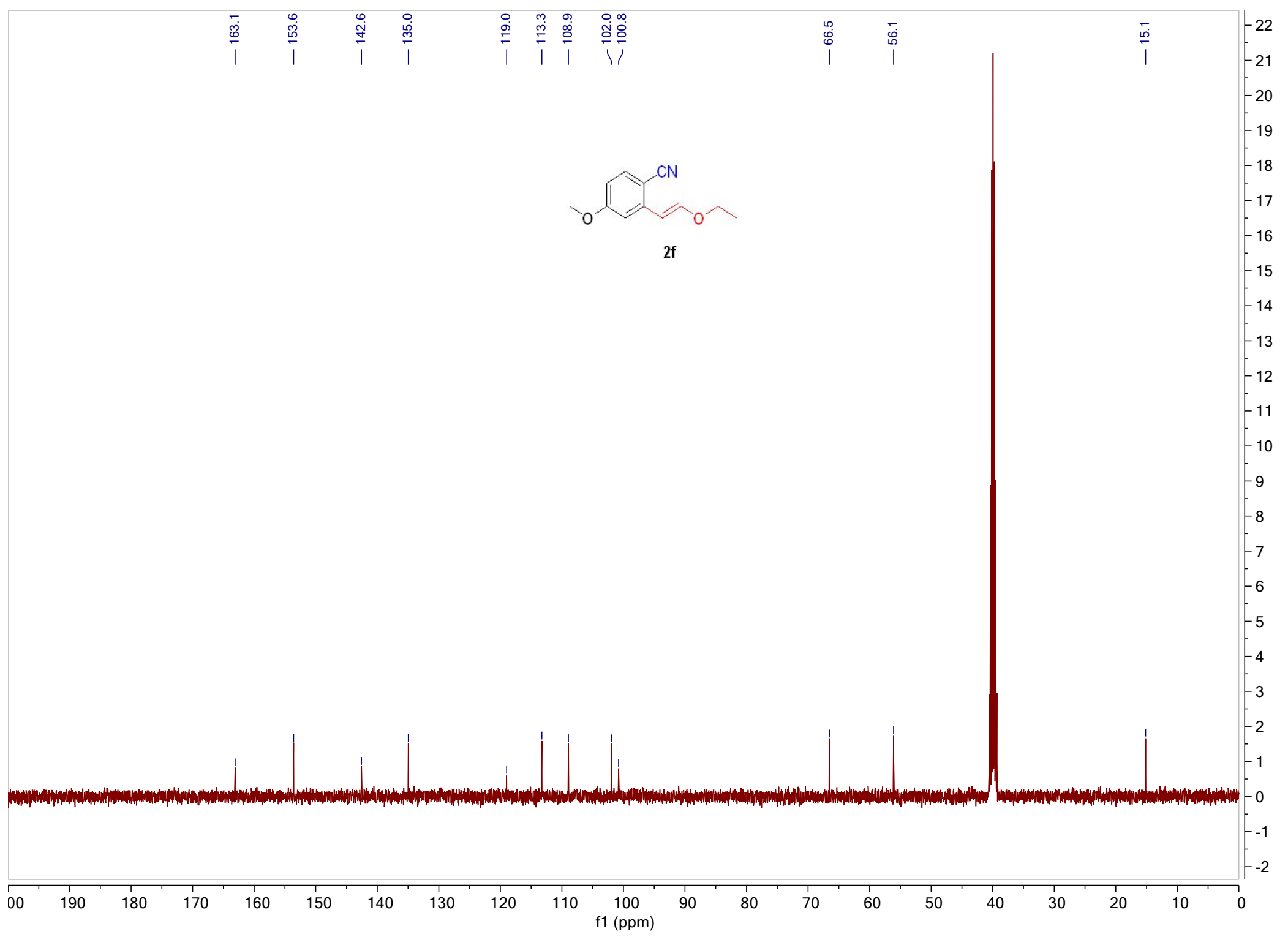

$\mathrm{SI}-13$ 
Figure S13. ${ }^{1} \mathrm{H}-\mathrm{NMR}$ (600 MHz, DMSO-6d) for compound $2 \mathrm{~g}$.

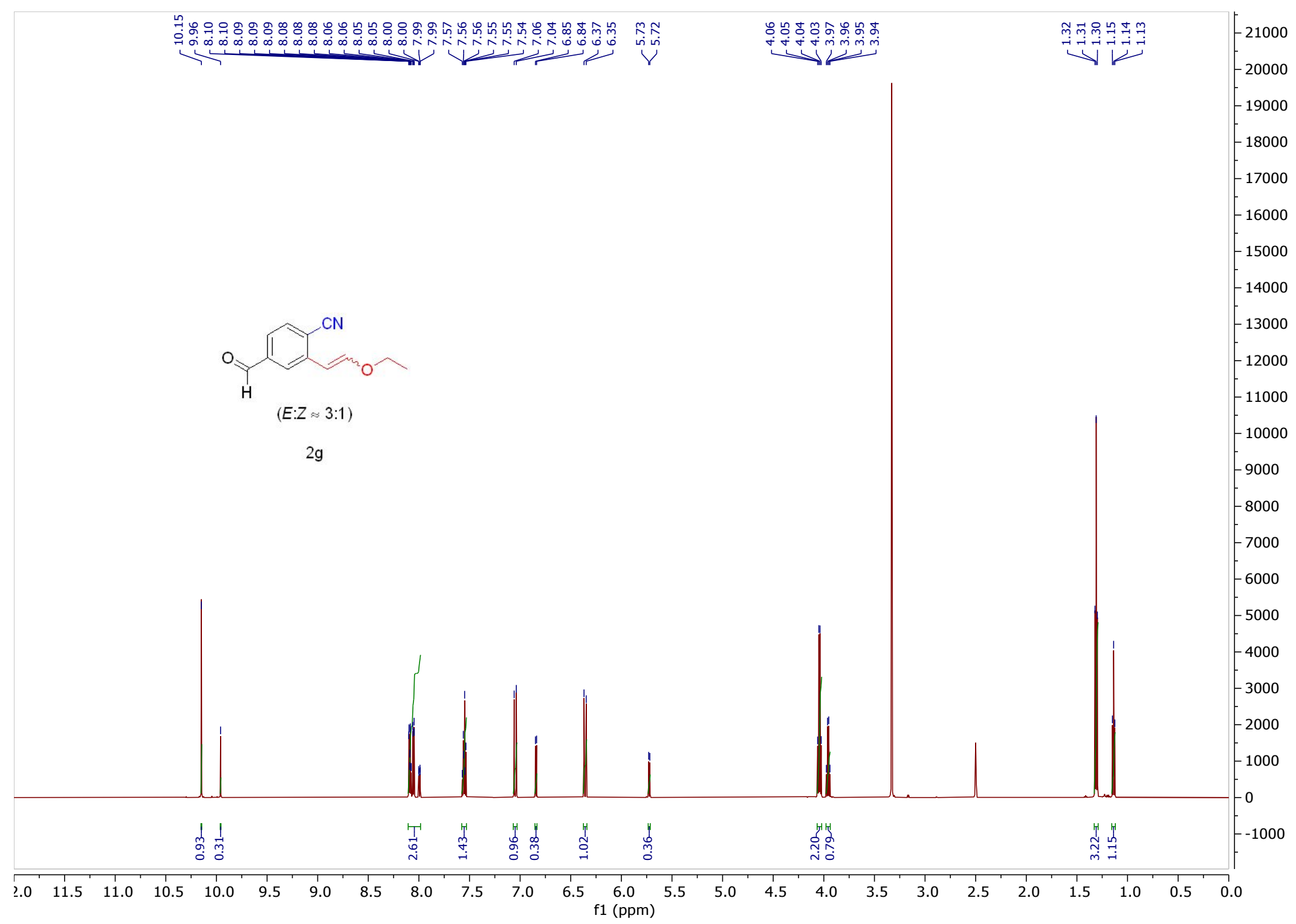


Figure $\mathrm{S} 14 .{ }^{13} \mathrm{C}\left\{{ }^{1} \mathrm{H}\right\}-\mathrm{NMR}\left(151 \mathrm{MHz}, \mathrm{CDCl}_{3}\right)$ for compound $2 \mathrm{~g}$.

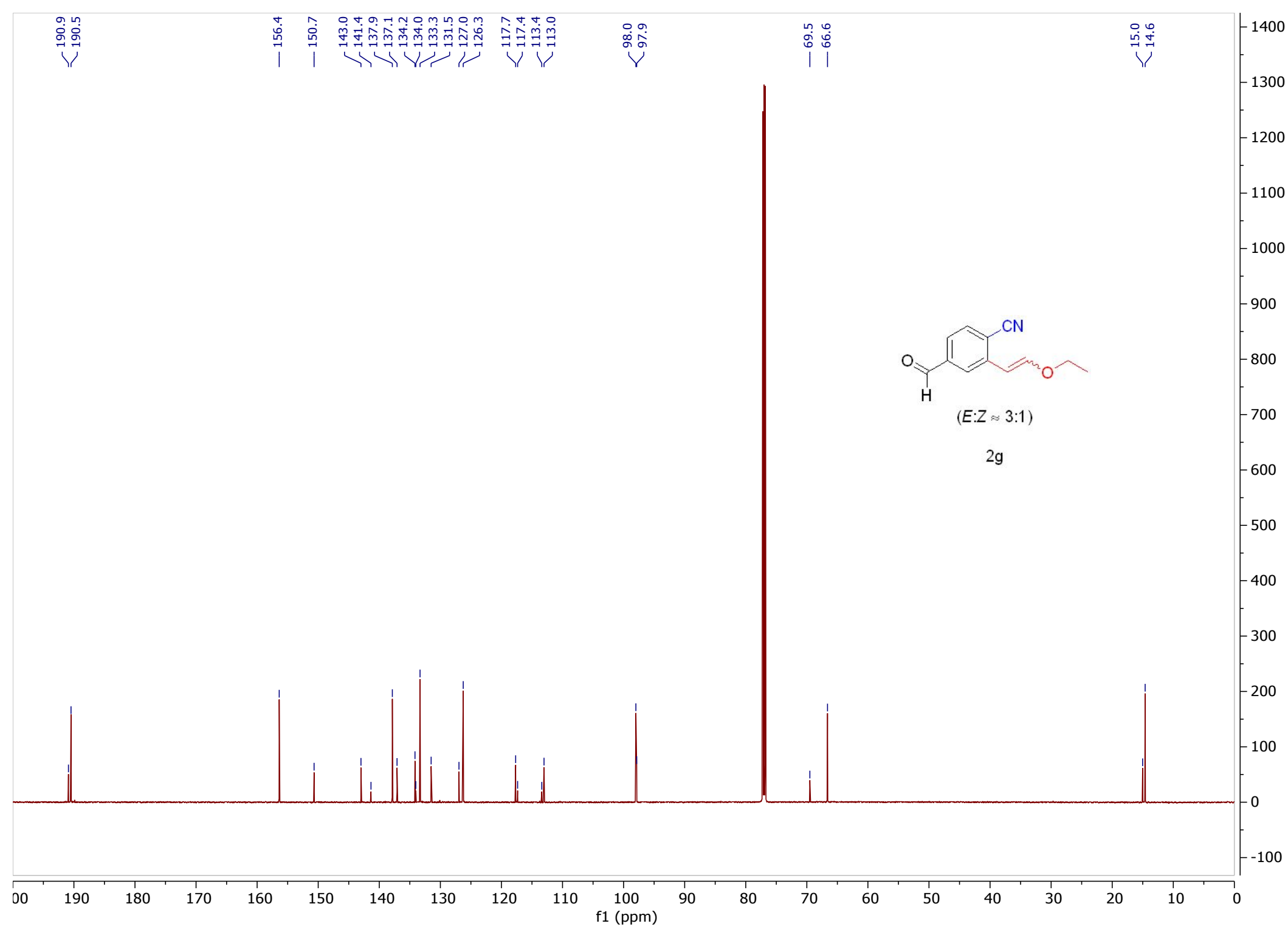


Figure S15. ${ }^{1} \mathrm{H}-\mathrm{NMR}\left(400 \mathrm{MHz}, \mathrm{CDCl}_{3}\right)$ for compound $2 \mathrm{~h}$.

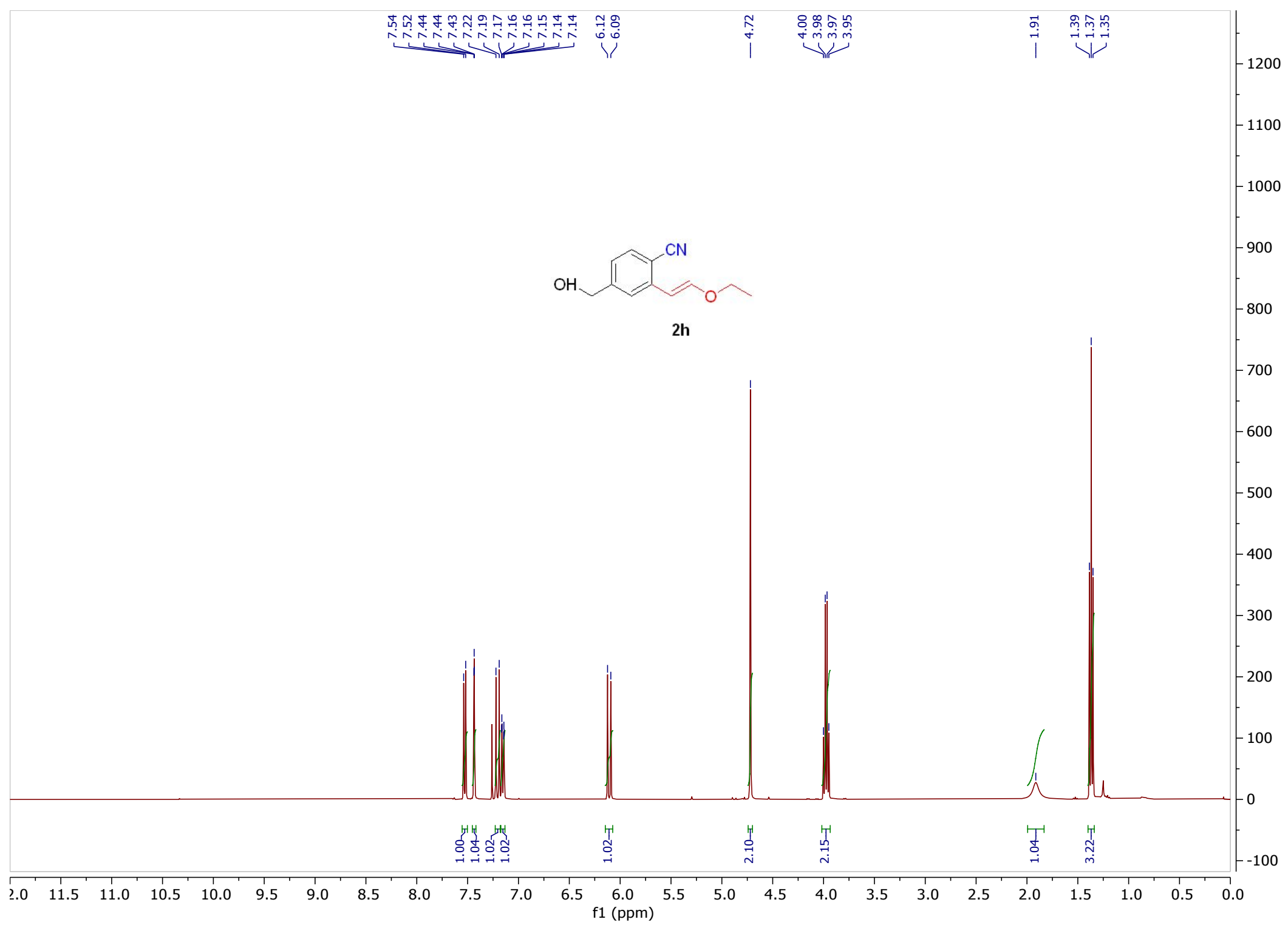

SI-16 
Figure S16. ${ }^{13} \mathrm{C}\left\{{ }^{1} \mathrm{H}\right\}-\mathrm{NMR}\left(101 \mathrm{MHz}, \mathrm{CDCl}_{3}\right)$ for compound $2 \mathrm{~h}$.

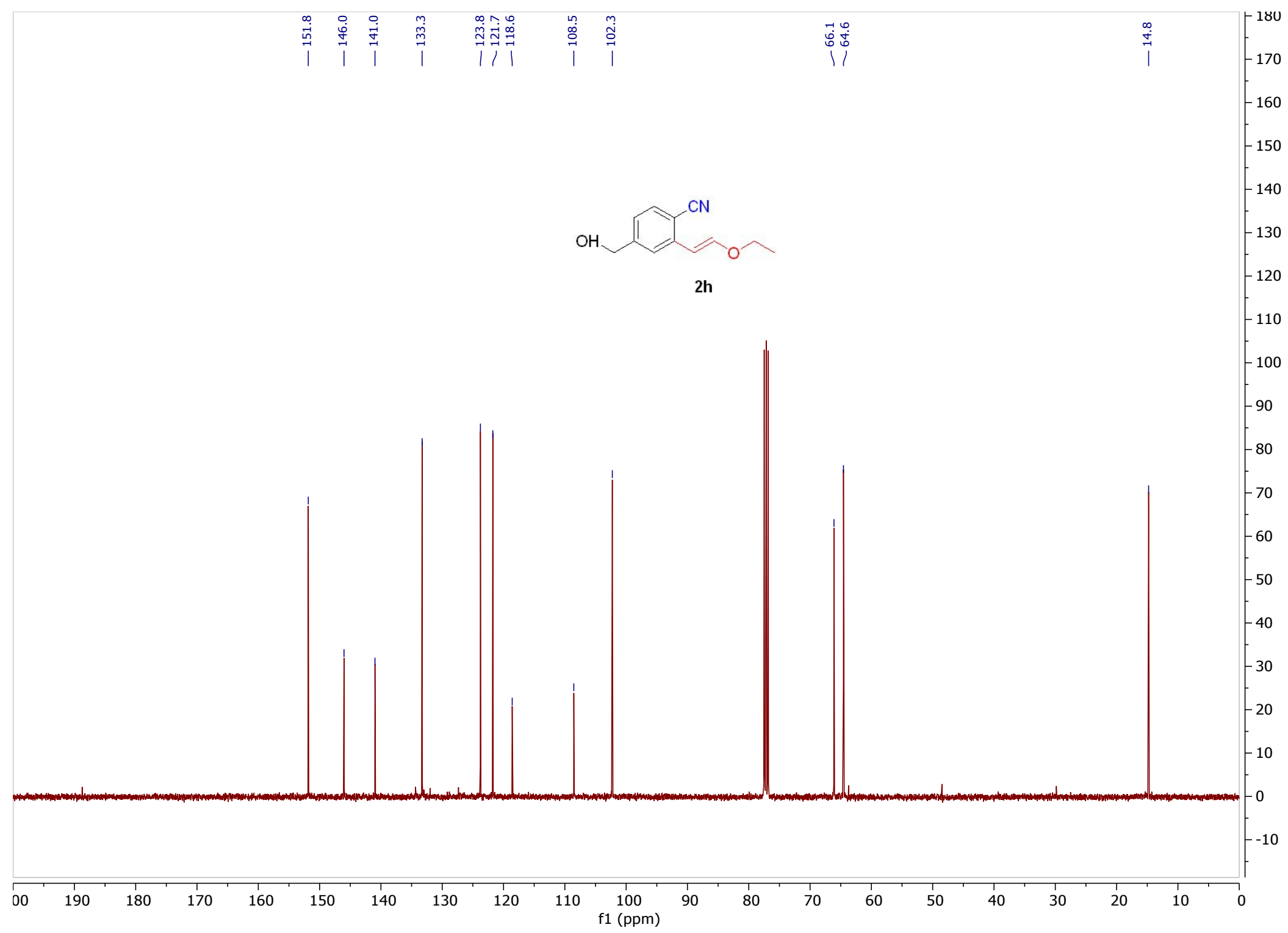


Figure S17. ${ }^{1} \mathrm{H}-\mathrm{NMR}$ (600 MHz, DMSO-d6) for compound $2 \mathrm{i}$.

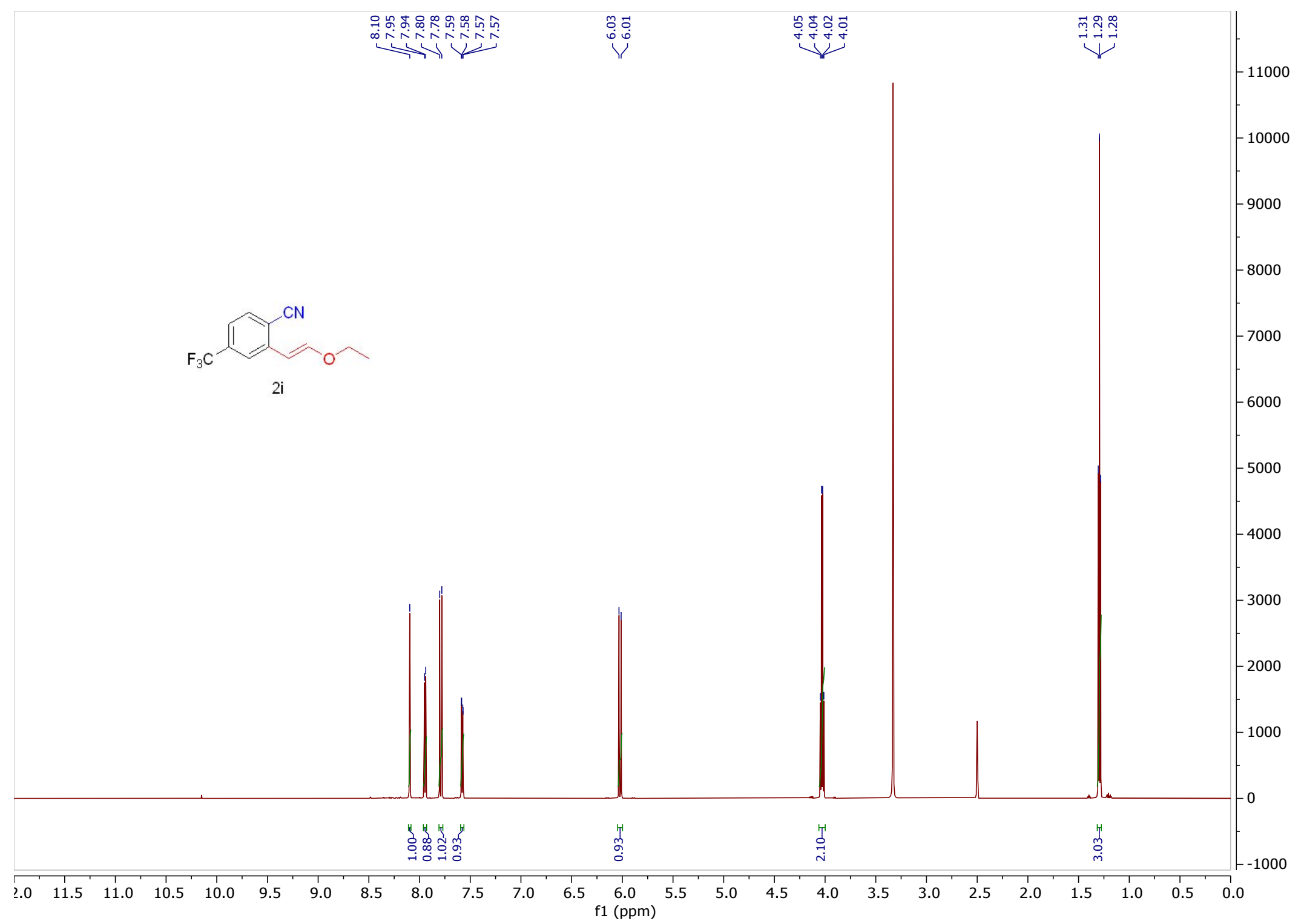


Figure S18. ${ }^{13} \mathrm{C}\left\{{ }^{1} \mathrm{H}\right\}-\mathrm{NMR}$ (151 MHz, DMSO-d6) for compound $2 \mathrm{i}$.

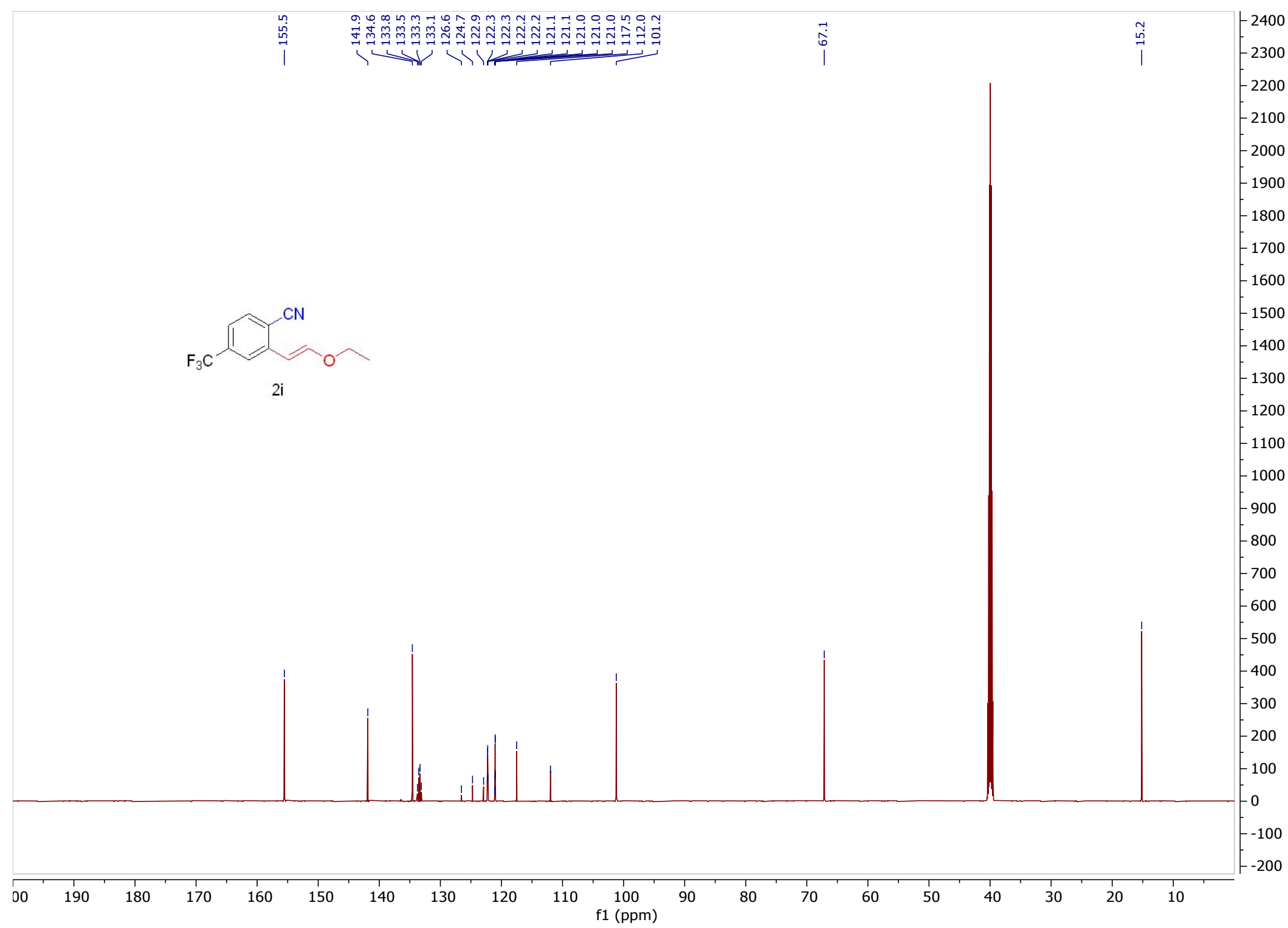


Figure S19. ${ }^{19} \mathrm{~F}-\mathrm{NMR}$ (376 MHz, DMSO-6d) for compound $2 \mathrm{i}$.

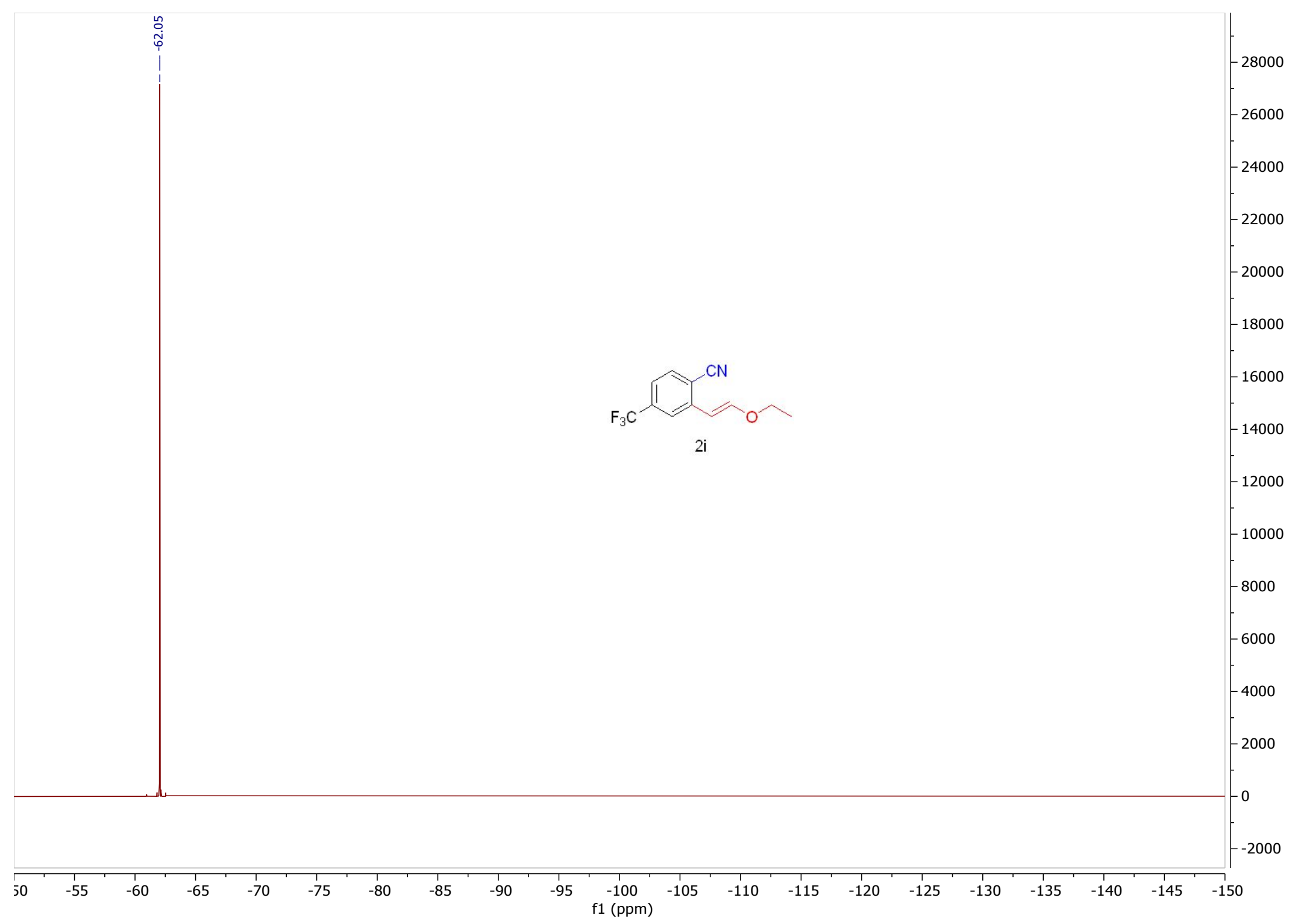


Figure S20. ${ }^{1} \mathrm{H}-\mathrm{NMR}\left(400 \mathrm{MHz}, \mathrm{CDCl}_{3}\right)$ for compound $2 \mathrm{j}$.

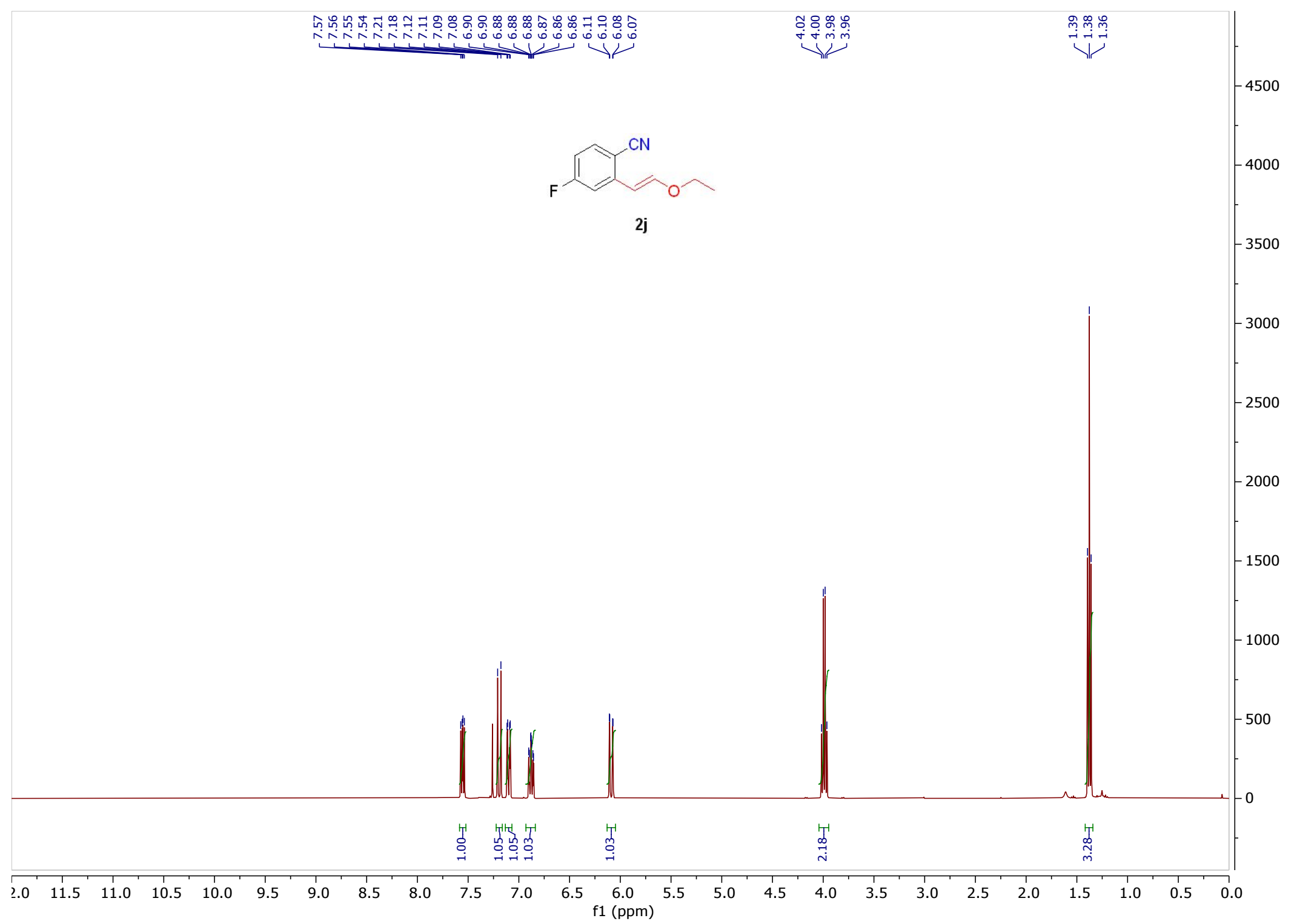


Figure S21. ${ }^{13} \mathrm{C}\left\{{ }^{1} \mathrm{H}\right\}-\mathrm{NMR}\left(101 \mathrm{MHz}, \mathrm{CDCl}_{3}\right)$ for compound $2 \mathrm{j}$.

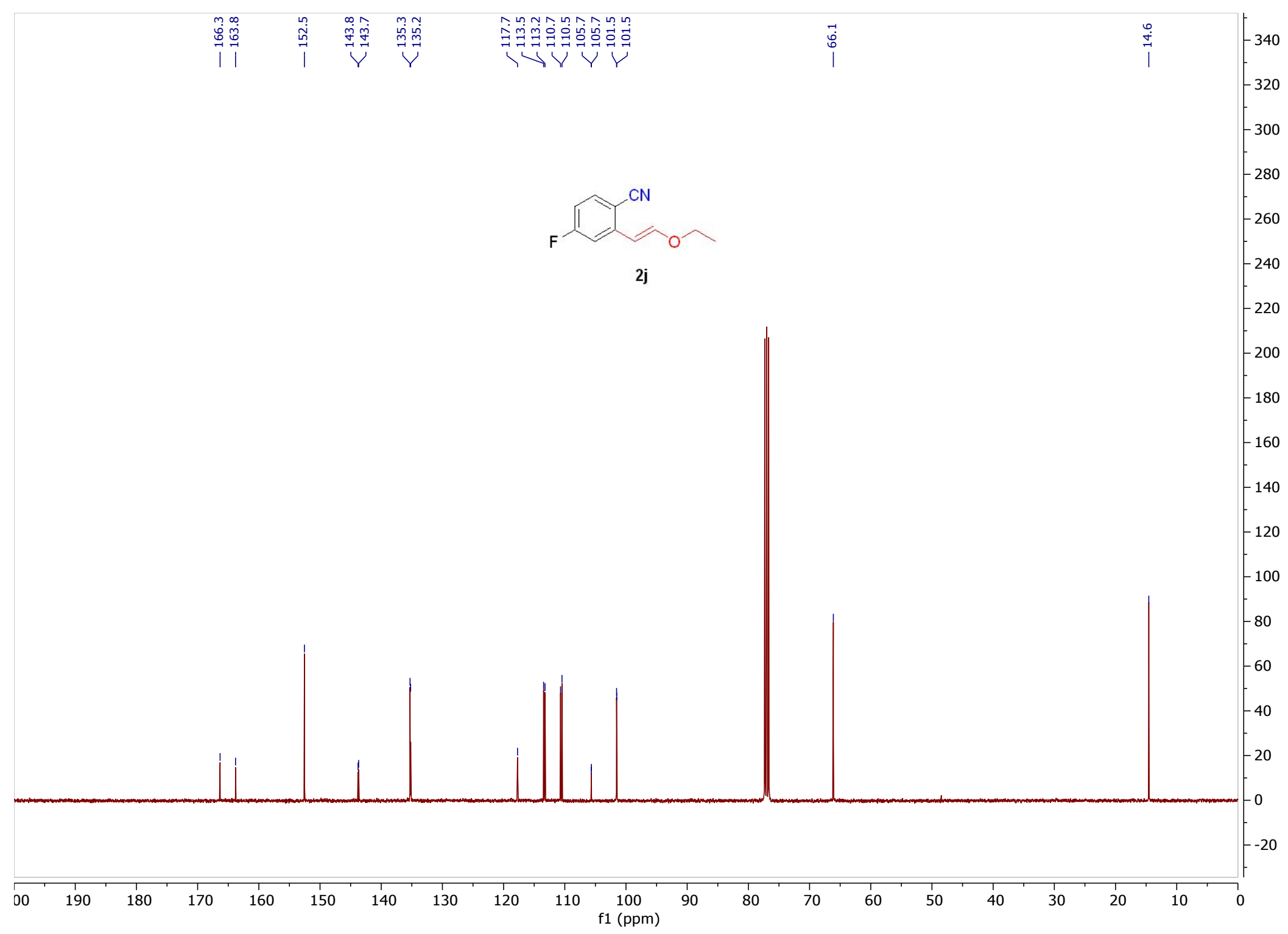


Figure S22. ${ }^{19} \mathrm{~F}-\mathrm{NMR}\left(471 \mathrm{MHz}, \mathrm{CDCl}_{3}\right)$ for compound $2 \mathrm{j}$.

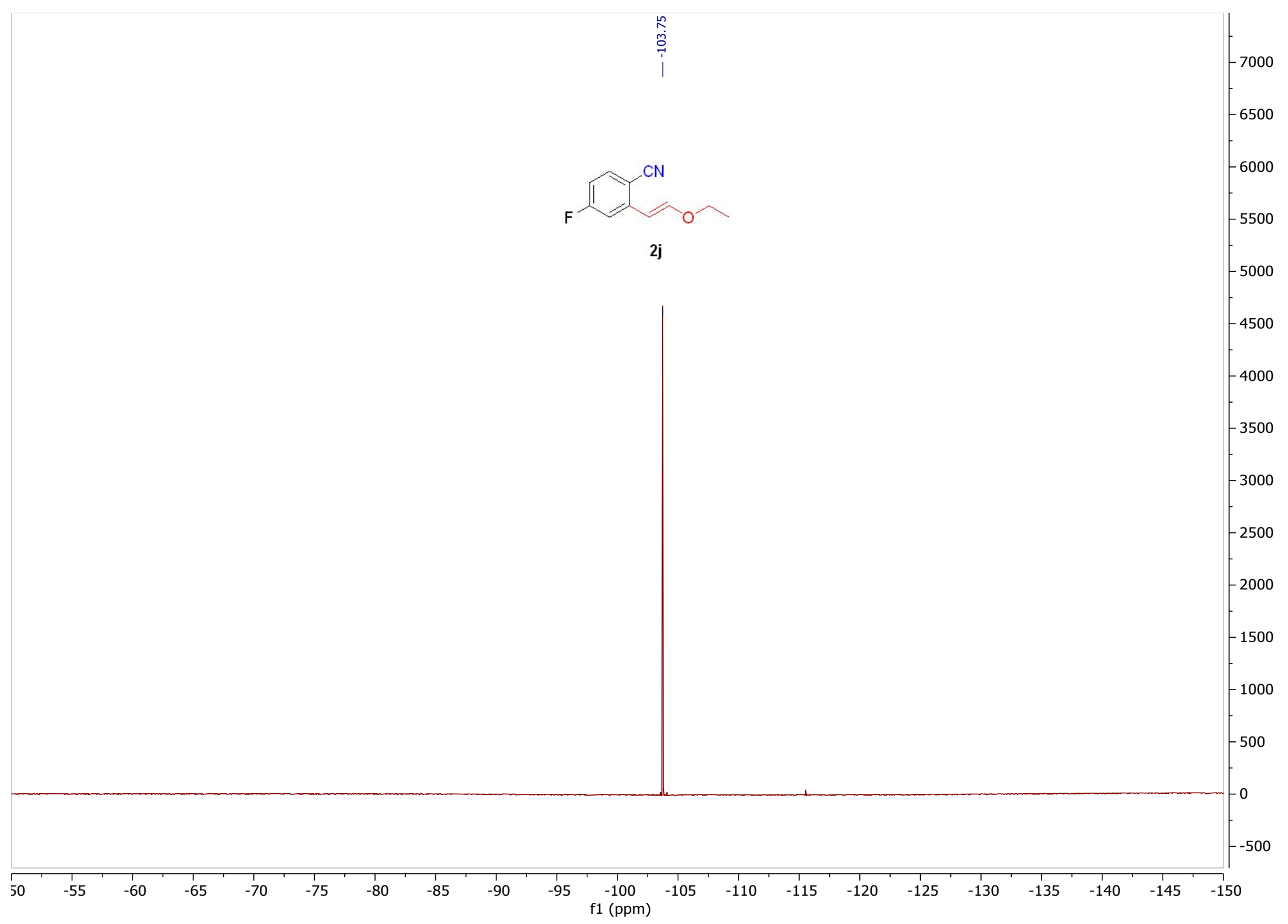


Figure S23. ${ }^{1} \mathrm{H}-\mathrm{NMR}\left(400 \mathrm{MHz}, \mathrm{CDCl}_{3}\right)$ for compound $2 \mathrm{k}$.

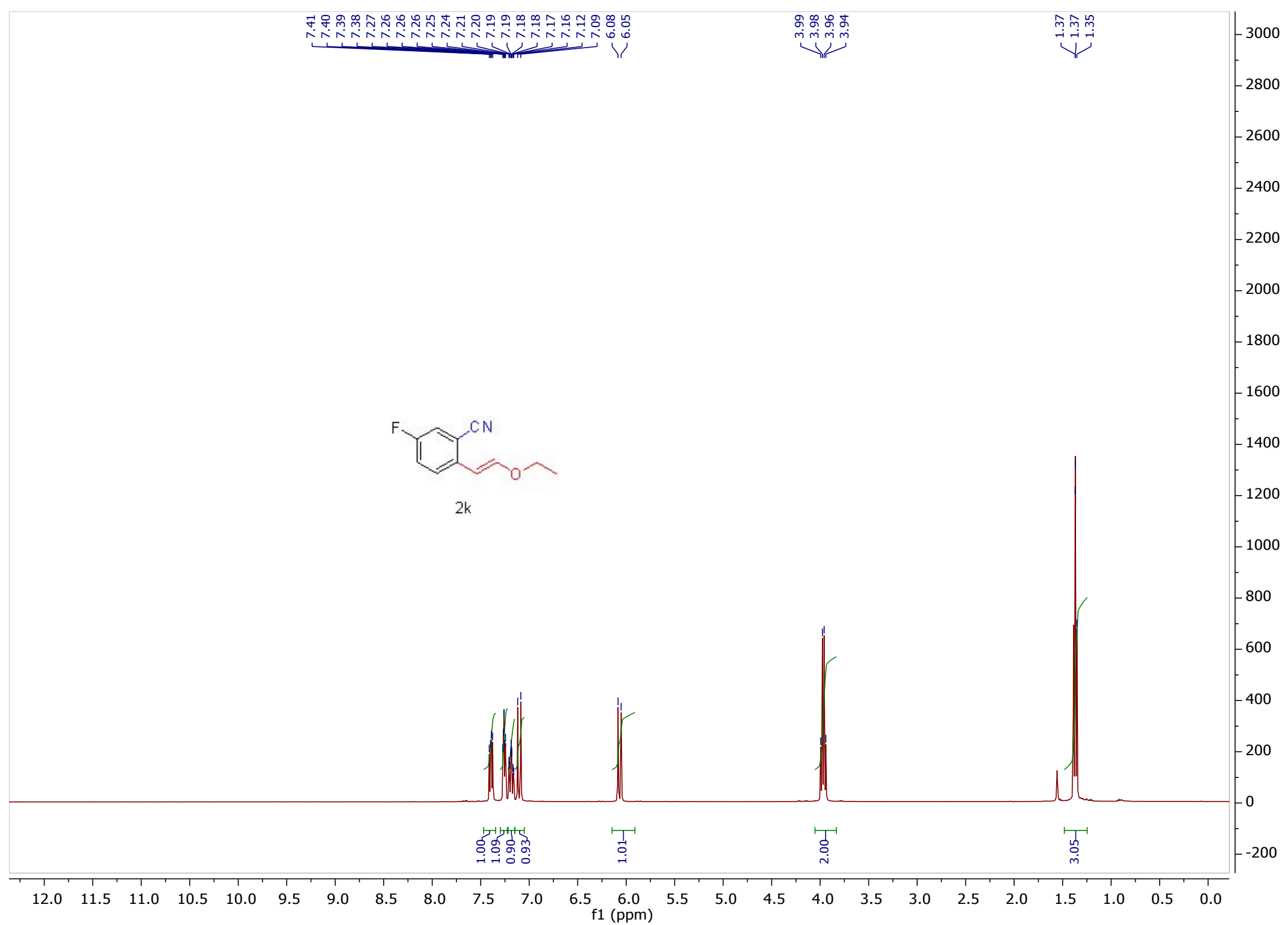


Figure S24. ${ }^{13} \mathrm{C}\left\{{ }^{1} \mathrm{H}\right\}-\mathrm{NMR}\left(151 \mathrm{MHz}, \mathrm{CDCl}_{3}\right)$ for compound $2 \mathrm{k}$.

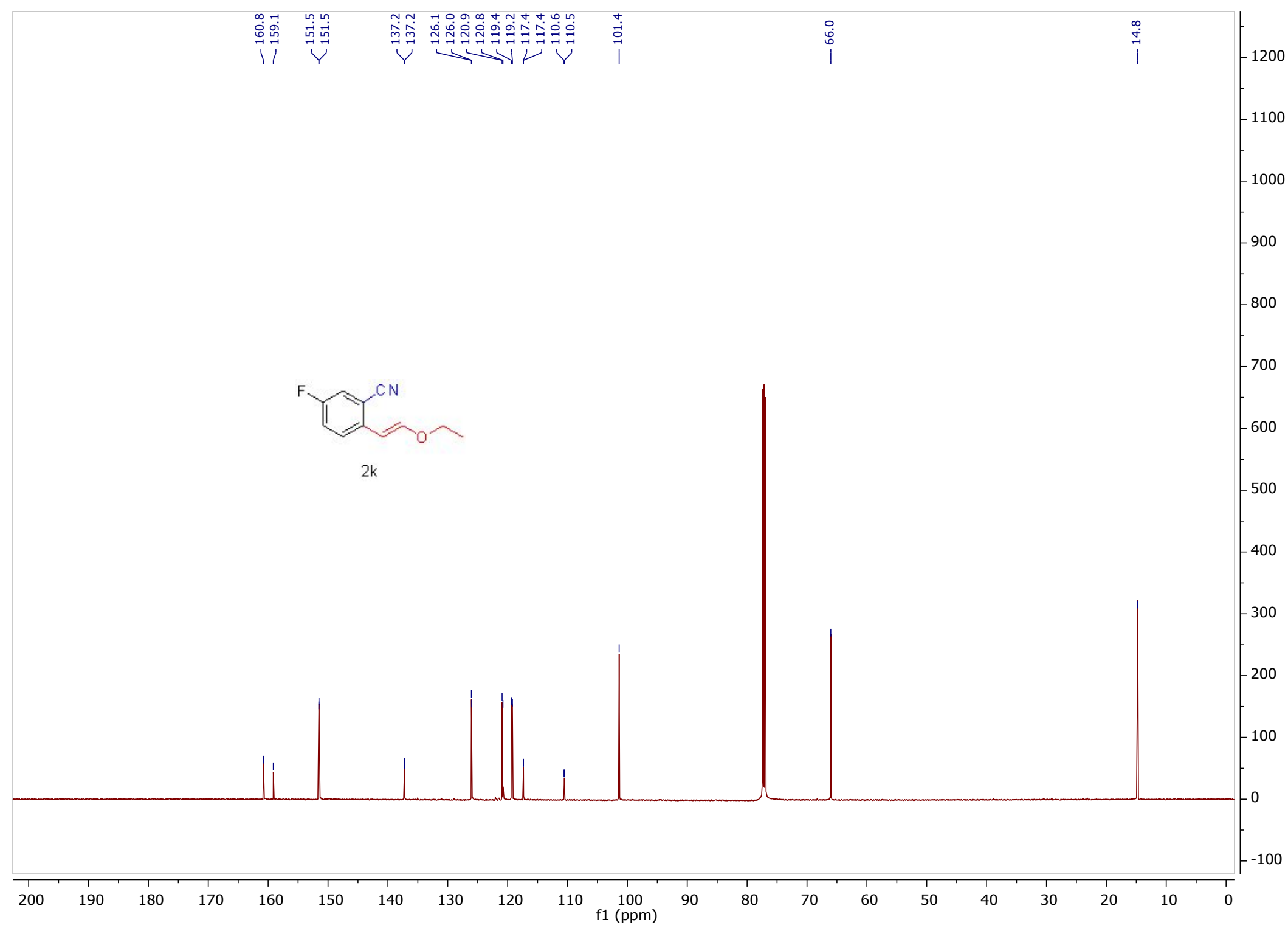


Figure S25. ${ }^{19} \mathrm{~F}-\mathrm{NMR}$ (471 MHz, DMSO-d6) for compound 2k.

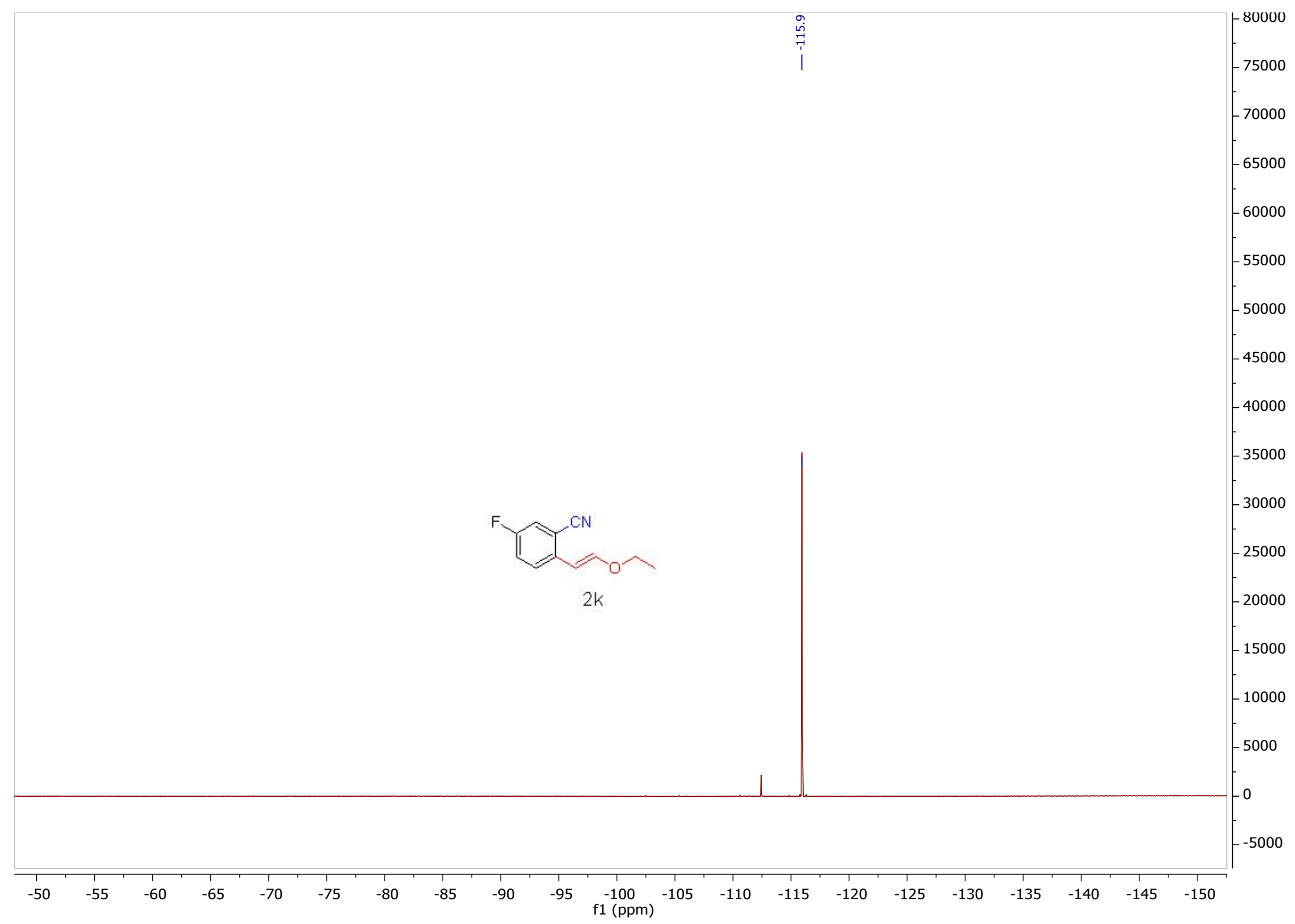


Figure S26. ${ }^{1} \mathrm{H}-\mathrm{NMR}$ (600 MHz, DMSO-d6) for compound $2 \mathrm{I}$

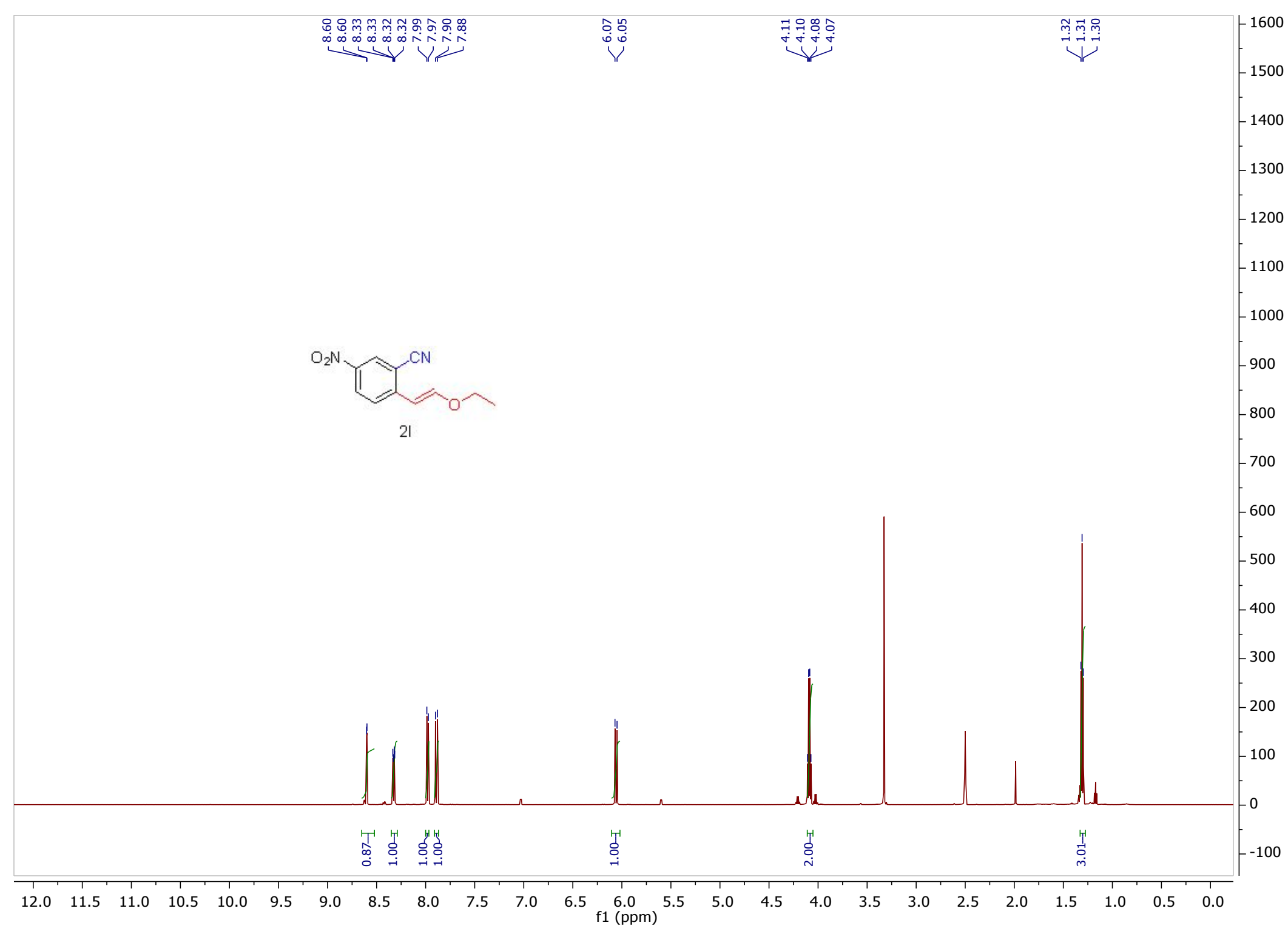


Figure S27. ${ }^{13} \mathrm{C}\left\{{ }^{1} \mathrm{H}\right\}-\mathrm{NMR}$ (151 MHz, DMSO-d6) for compound $2 \mathrm{I}$.

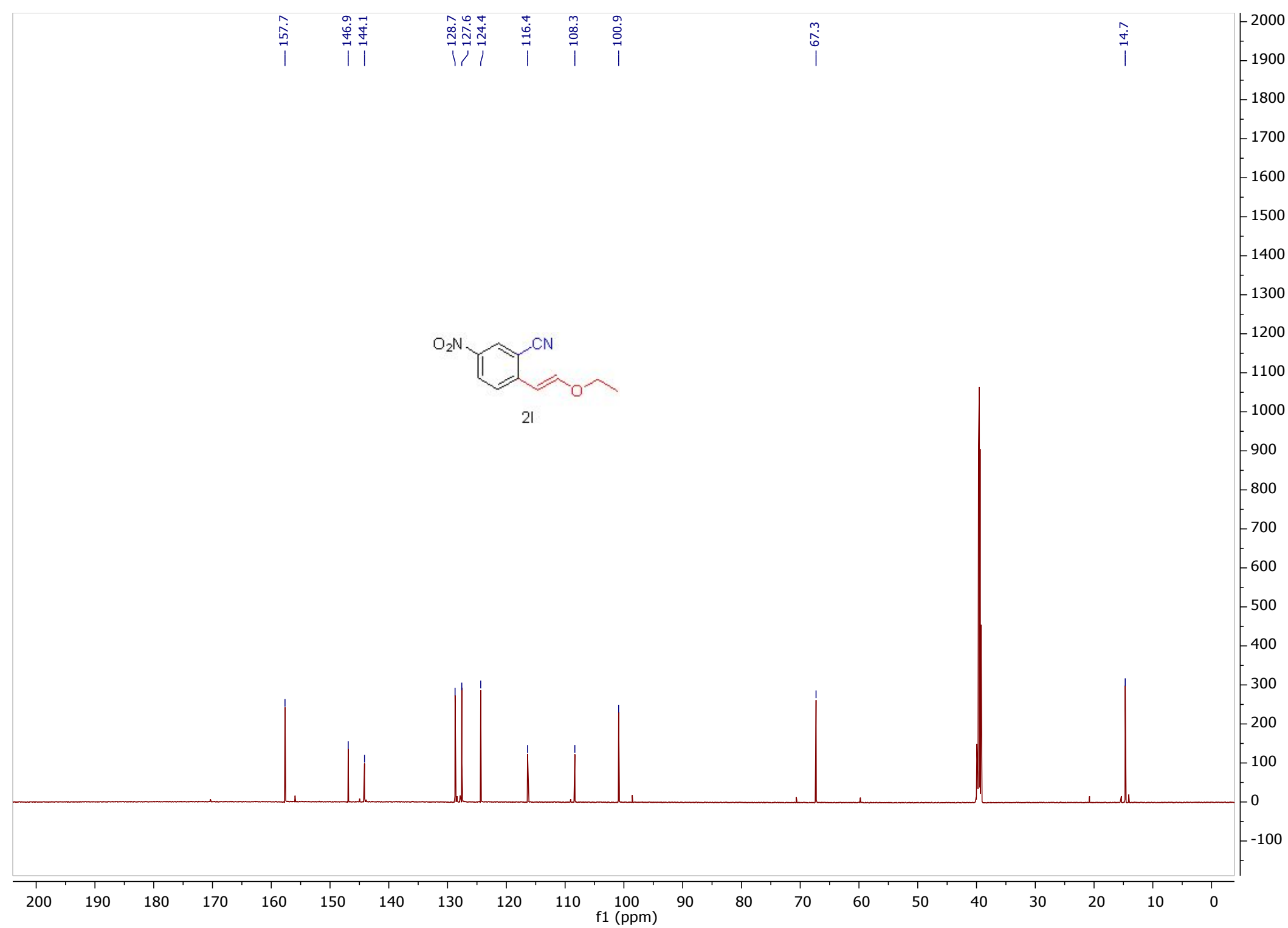


Figure S28. ${ }^{1} \mathrm{H}-\mathrm{NMR}\left(400 \mathrm{MHz}, \mathrm{CDCl}_{3}\right)$ for compound $2 \mathrm{~m}$

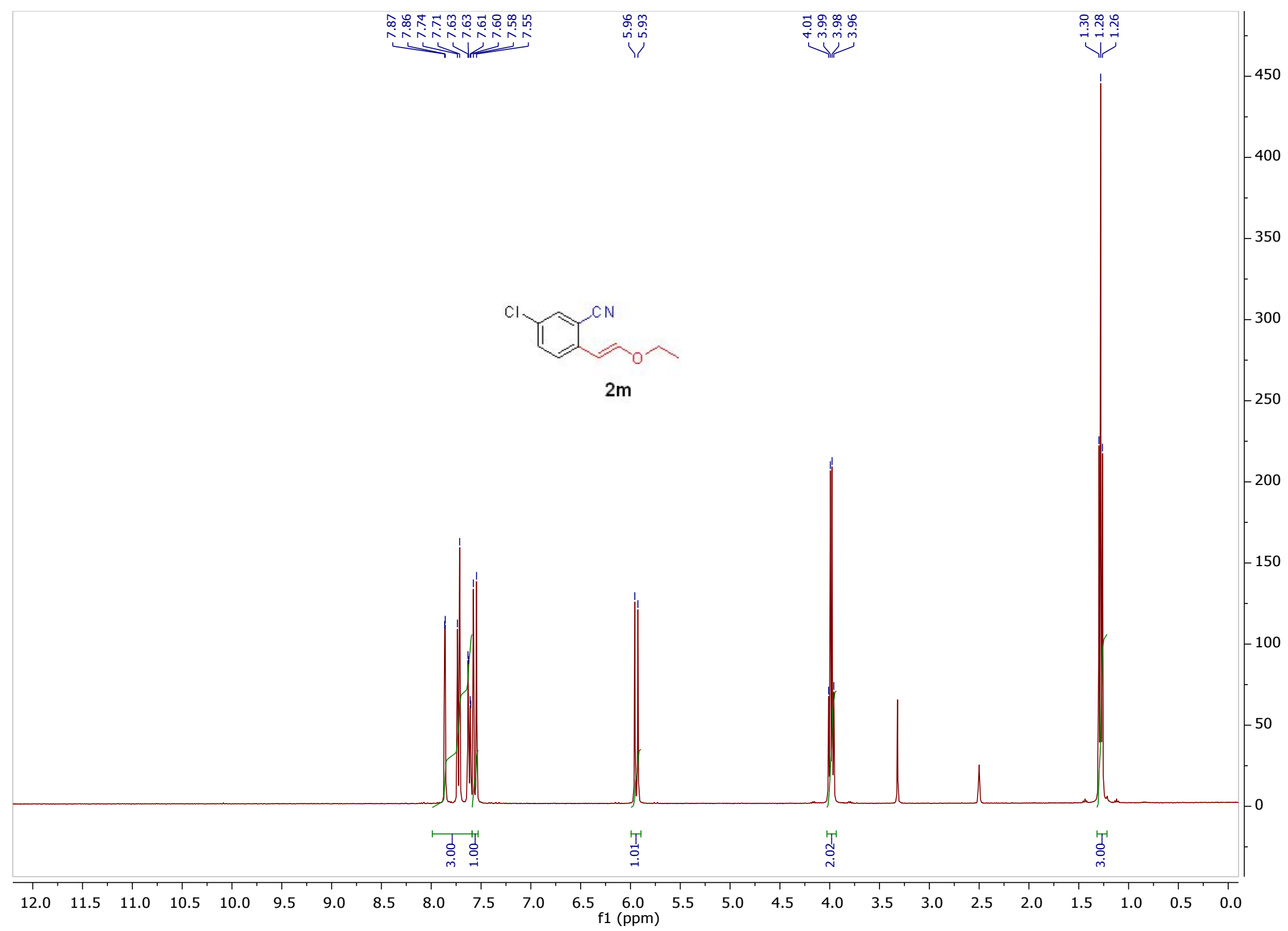


Figure S29. ${ }^{13} \mathrm{C}\left\{{ }^{1} \mathrm{H}\right\}-\mathrm{NMR}$ (101 MHz, DMSO-d6) for compound $2 \mathrm{~m}$

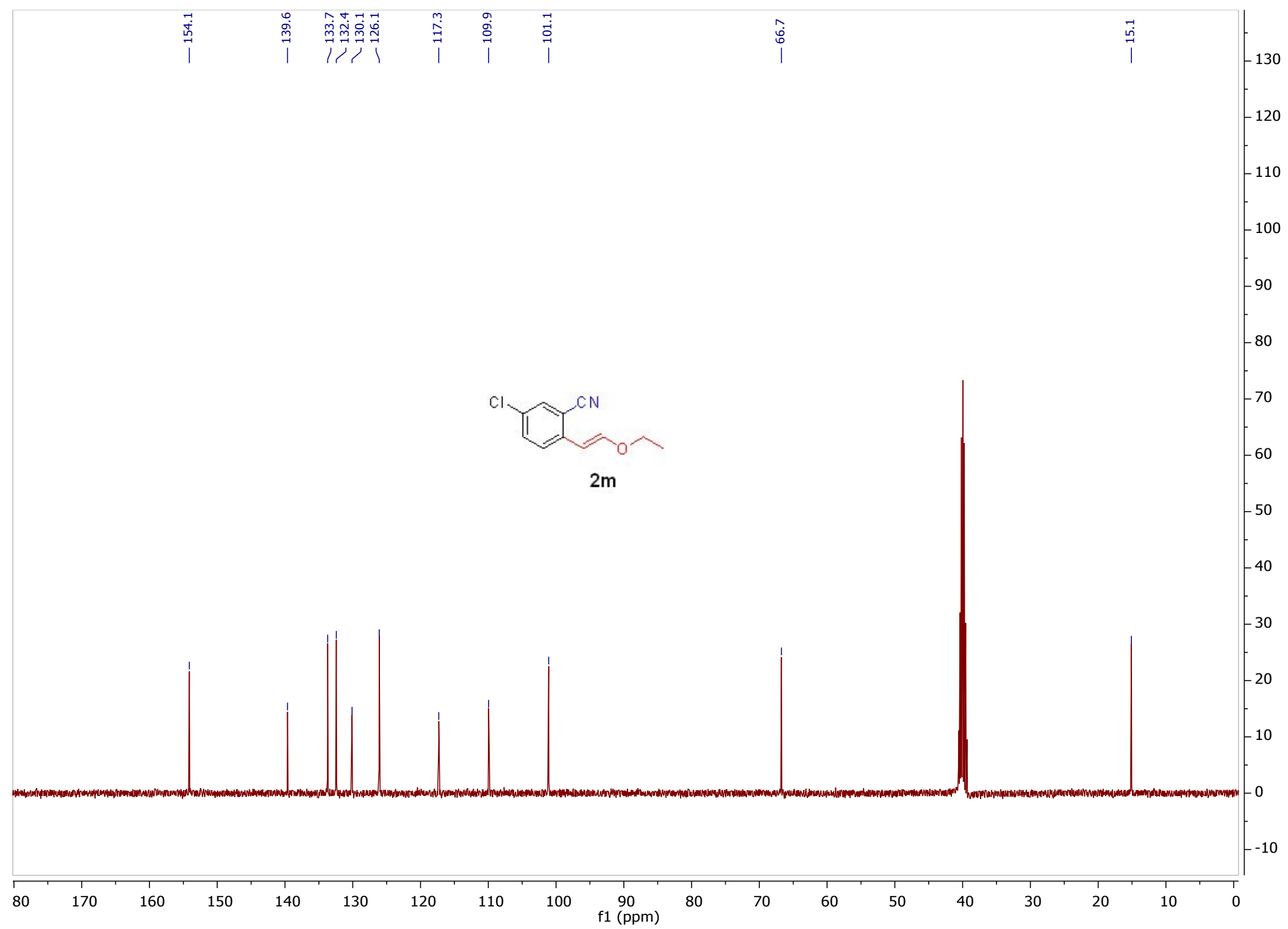


Figure S30. ${ }^{1} \mathrm{H}-\mathrm{NMR}$ (600 MHz, DMSO-d6) for compound $2 \mathrm{n}$

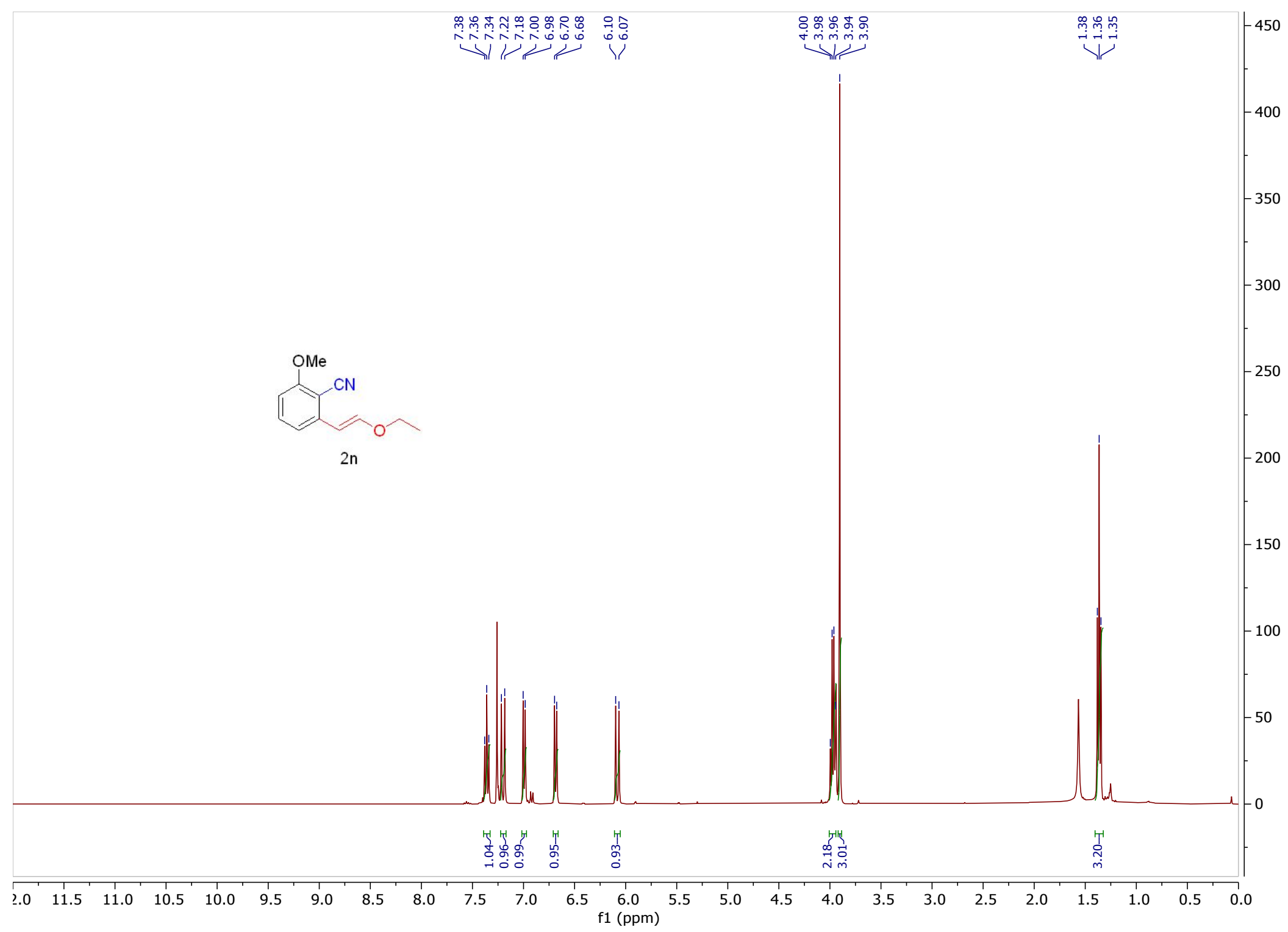


Figure S31. ${ }^{13} \mathrm{C}\left\{{ }^{1} \mathrm{H}\right\}-\mathrm{NMR}$ (101 MHz, DMSO-d6) for compound $2 \mathrm{n}$

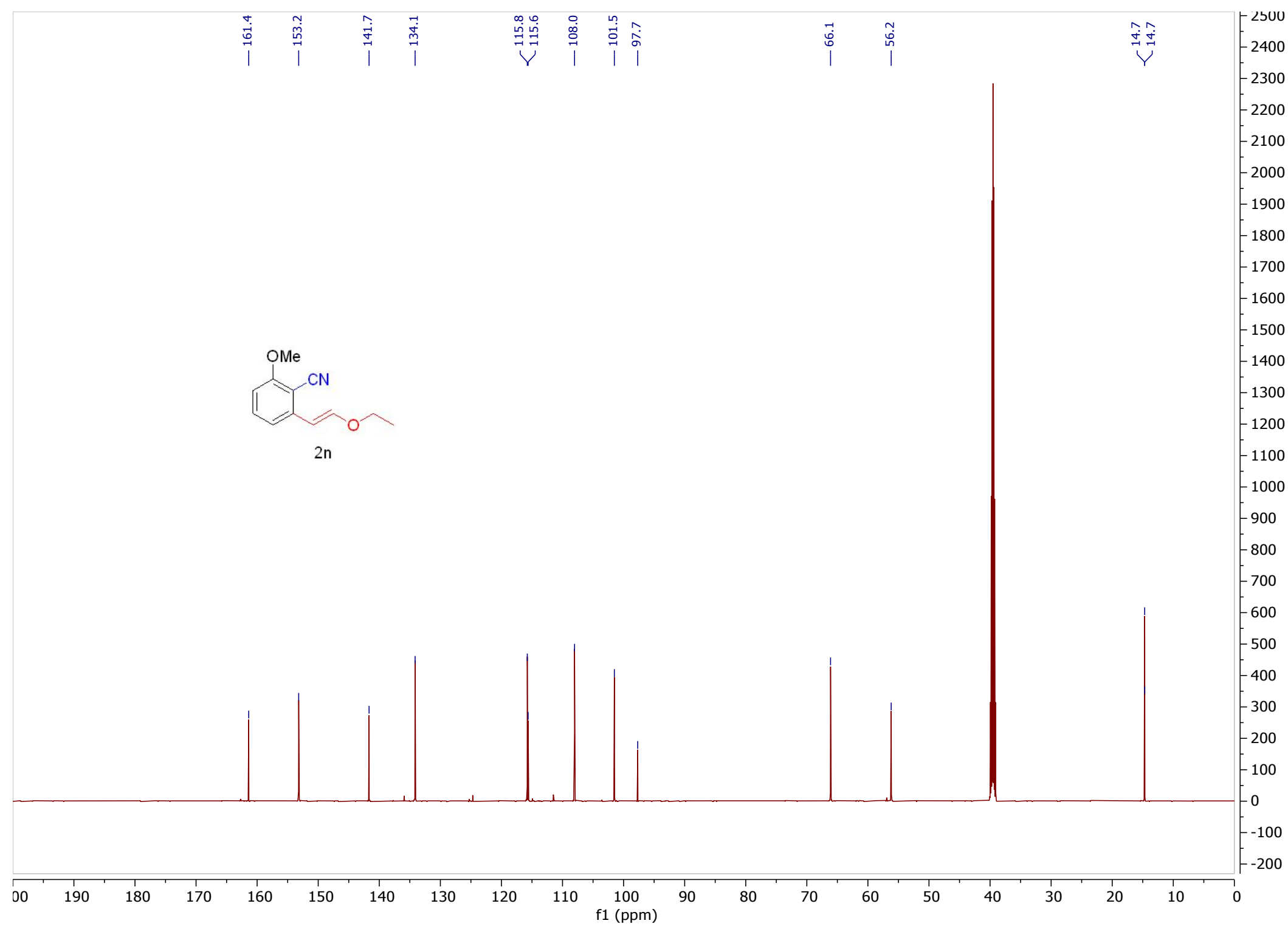


Figure S32. ${ }^{1} \mathrm{H}-\mathrm{NMR}$ (400 MHz, DMSO-d6) for compound 20.

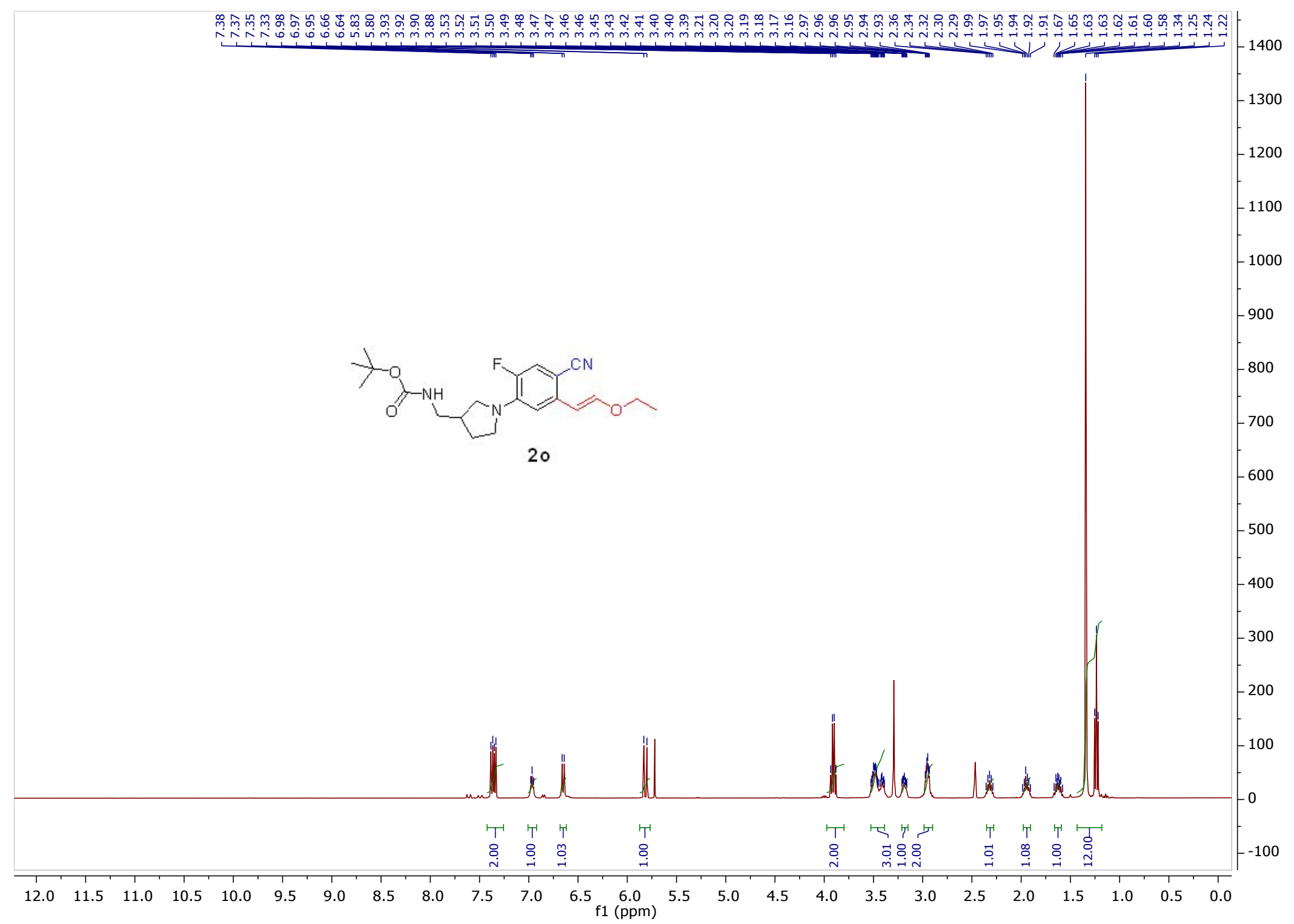


Figure S33. ${ }^{13} \mathrm{C}\left\{{ }^{1} \mathrm{H}\right\}-\mathrm{NMR}$ (101 MHz, DMSO-d6) for compound 20.

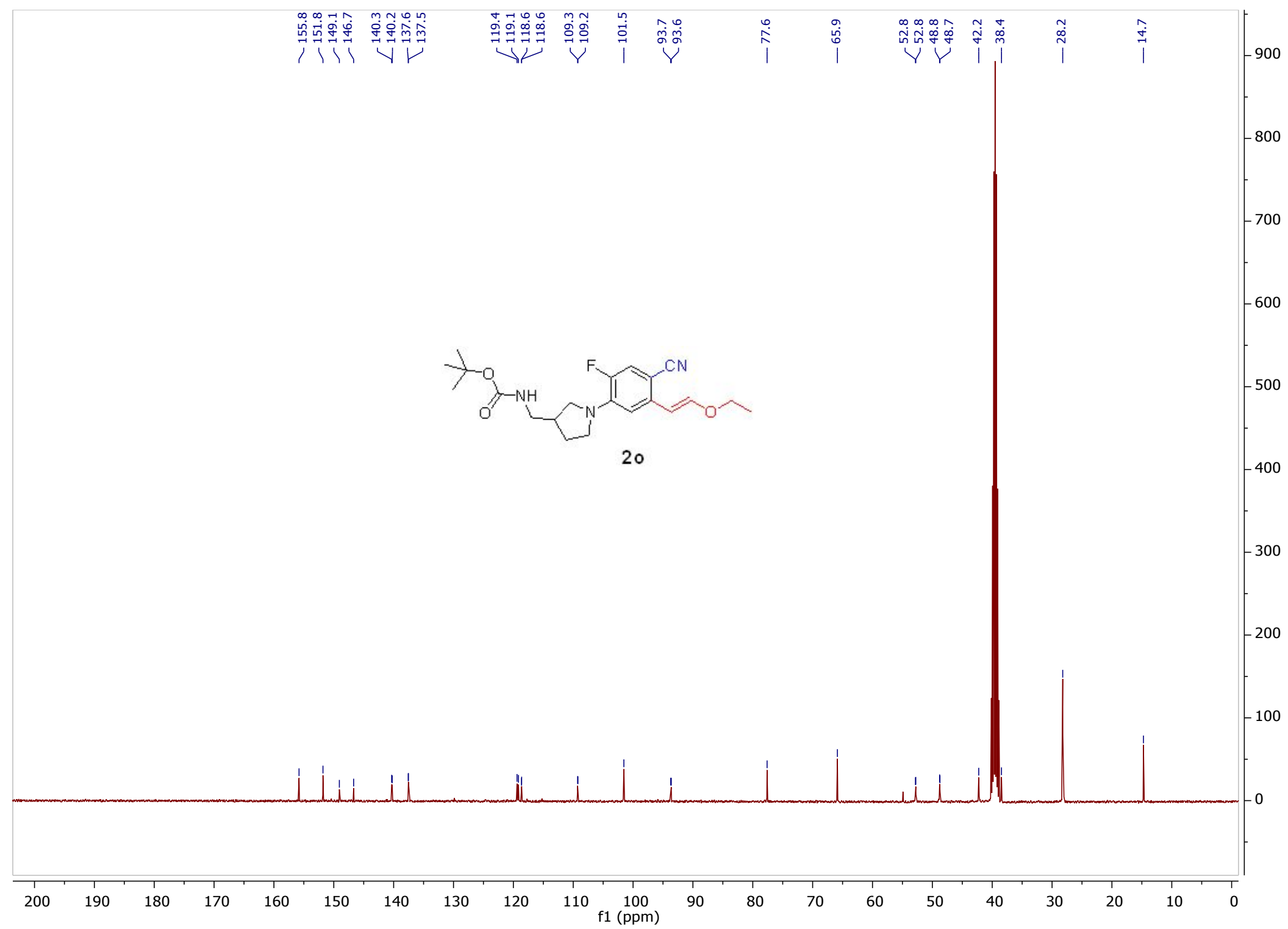


Figure S34. ${ }^{19} \mathrm{~F}-\mathrm{NMR}$ (376 MHz, DMSO-d6) for compound 20.

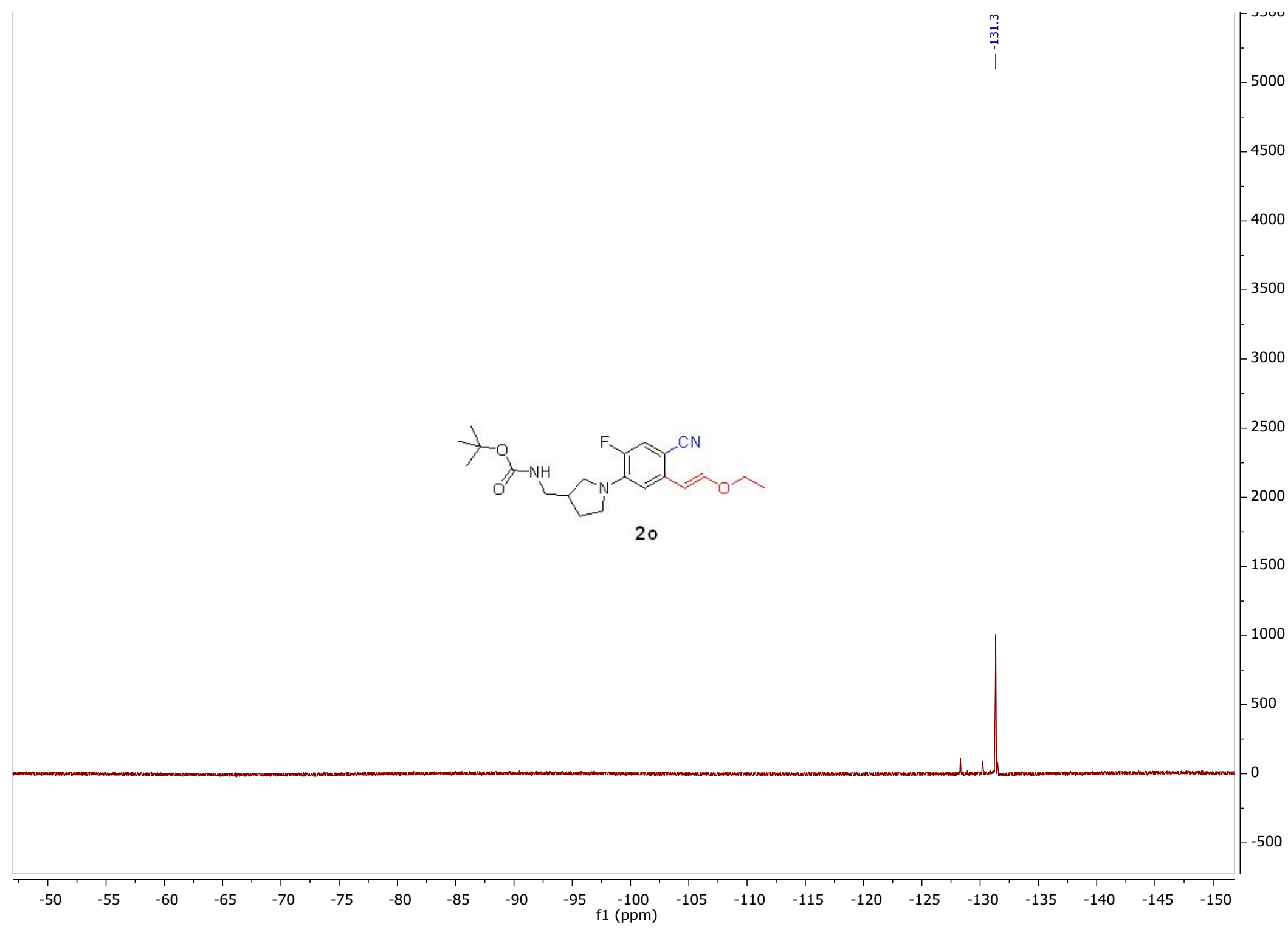


S2. NMR spectra for compounds 3a-3o.

Figure S35. ${ }^{1} \mathrm{H}-\mathrm{NMR}$ (400 MHz, DMSO-d6) for compound 3a.

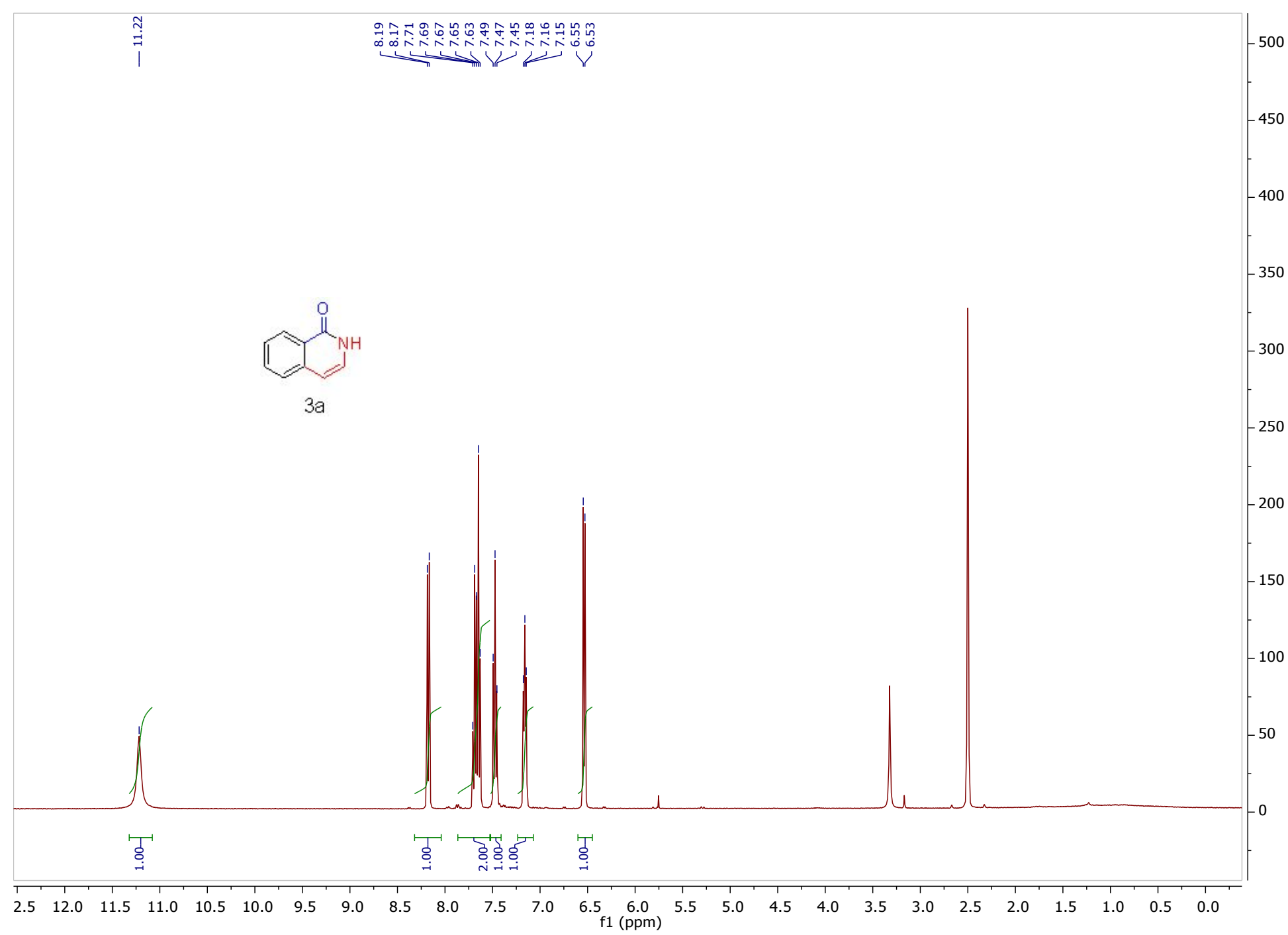


Figure S36. ${ }^{13} \mathrm{C}\left\{{ }^{1} \mathrm{H}\right\}-\mathrm{NMR}$ (101 MHz, DMSO-d6) for compound 3a.

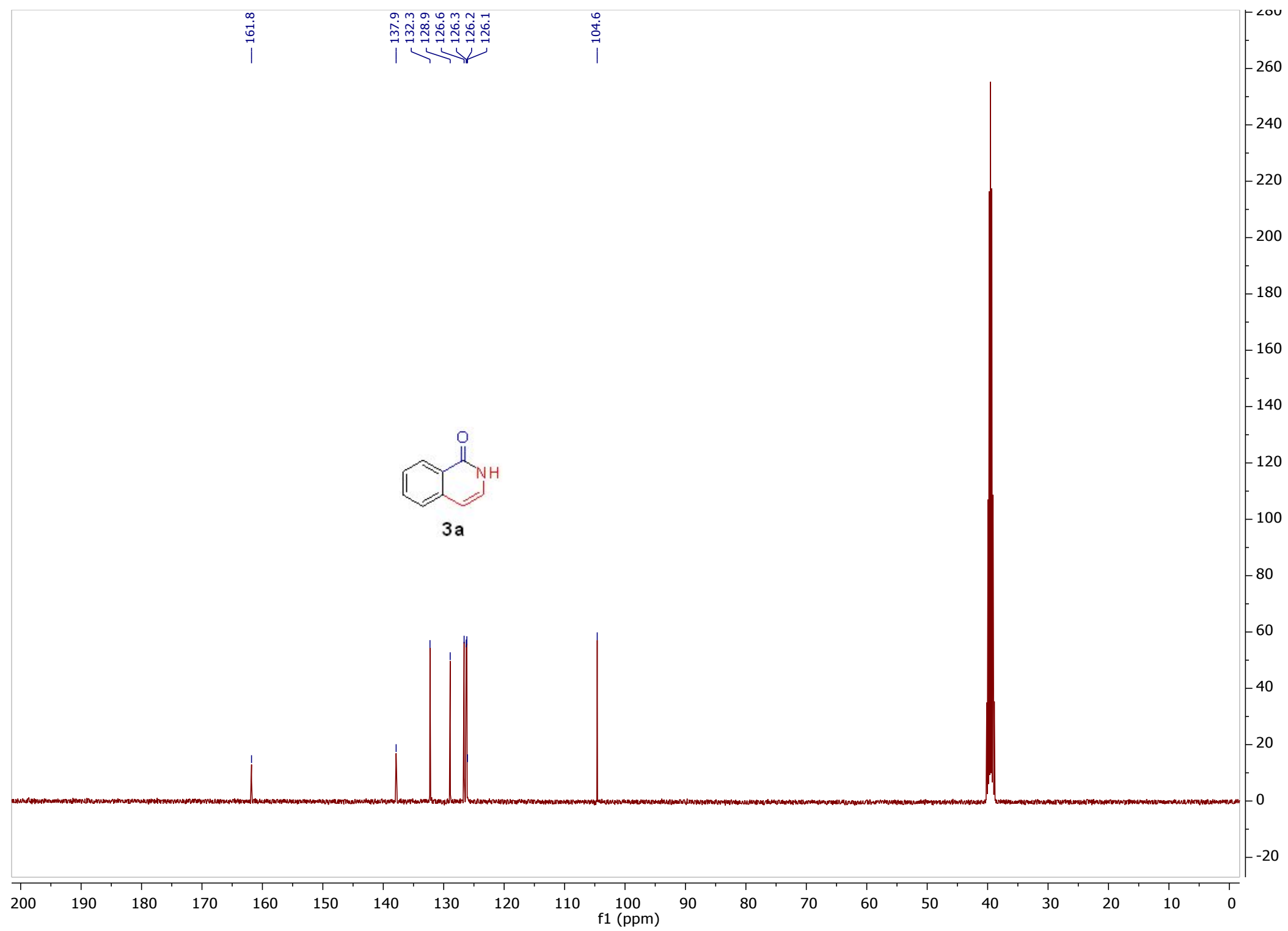


Figure S37. ${ }^{1} \mathrm{H}-\mathrm{NMR}(400 \mathrm{MHz}$, DMSO-d6) for compound $3 \mathrm{~b}$.

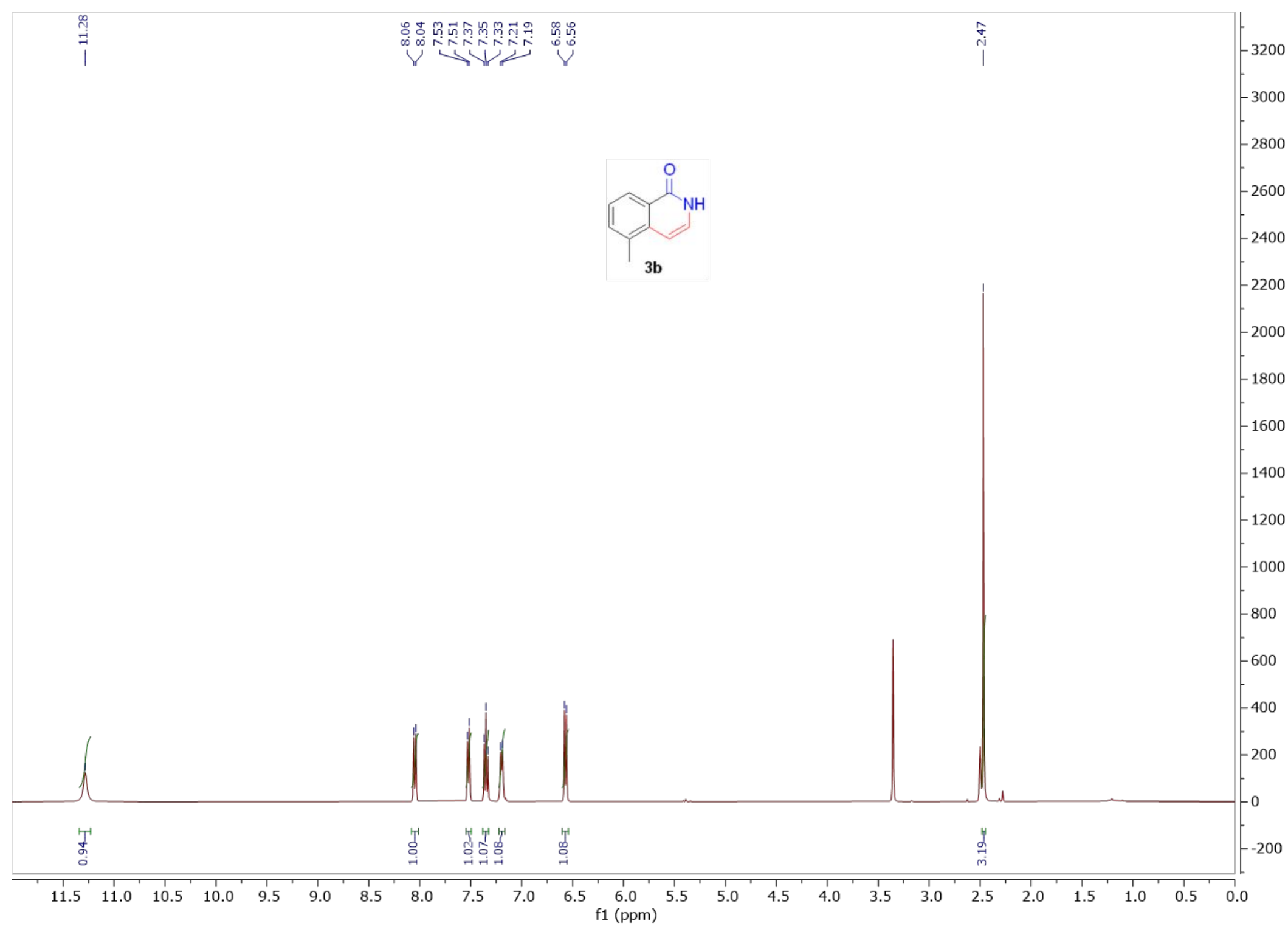


Figure S38. ${ }^{13} \mathrm{C}\left\{{ }^{1} \mathrm{H}\right\}-\mathrm{NMR}$ (101 MHz, DMSO-d6) for compound $3 \mathrm{~b}$.

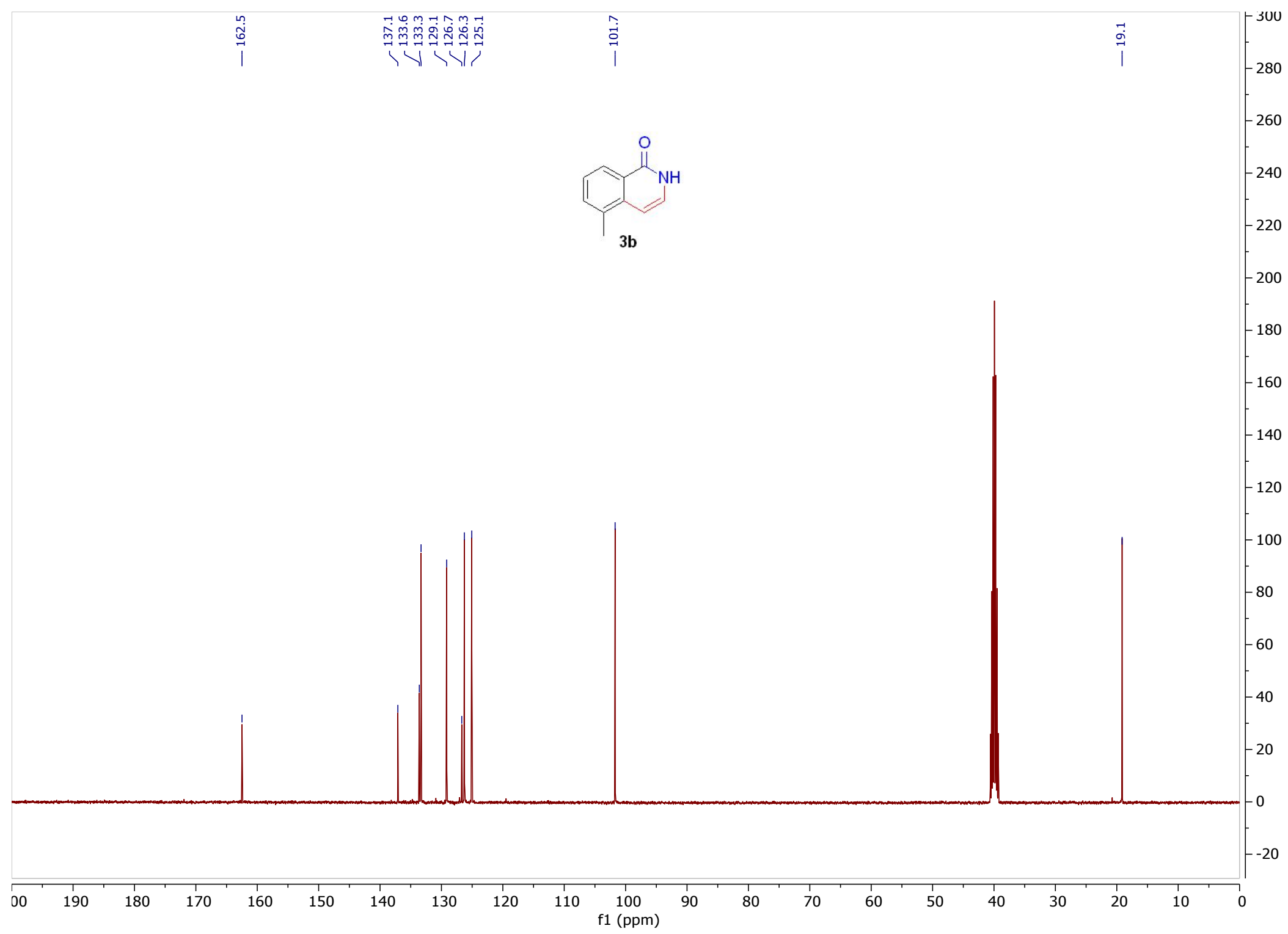


Figure S39. ${ }^{1} \mathrm{H}-\mathrm{NMR}$ (400 MHz, DMSO-d6) for compound 3c.

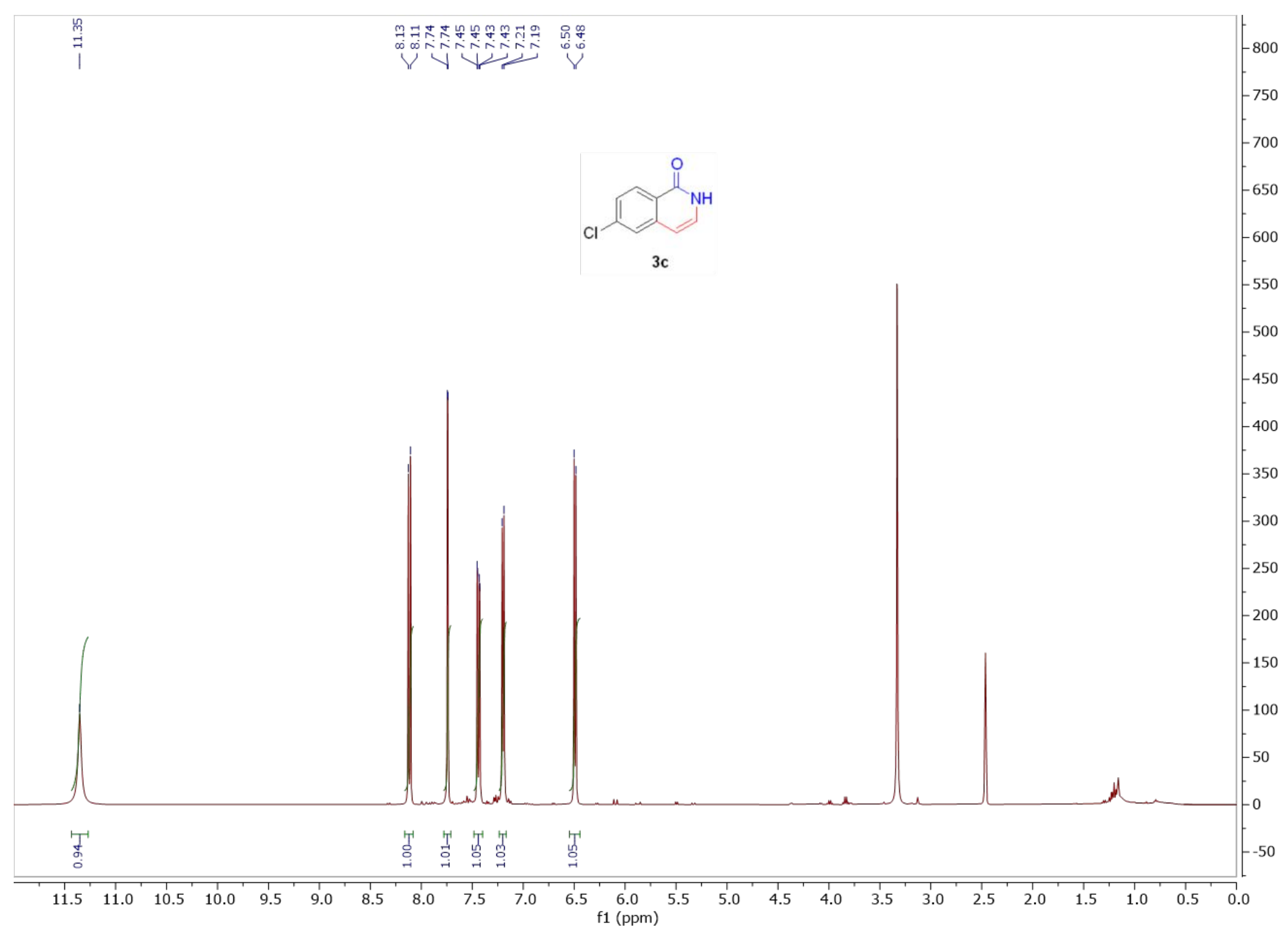


Figure S40. ${ }^{13} \mathrm{C}\left\{{ }^{1} \mathrm{H}\right\}-\mathrm{NMR}$ (101 MHz, DMSO-d6) for compound 3c.

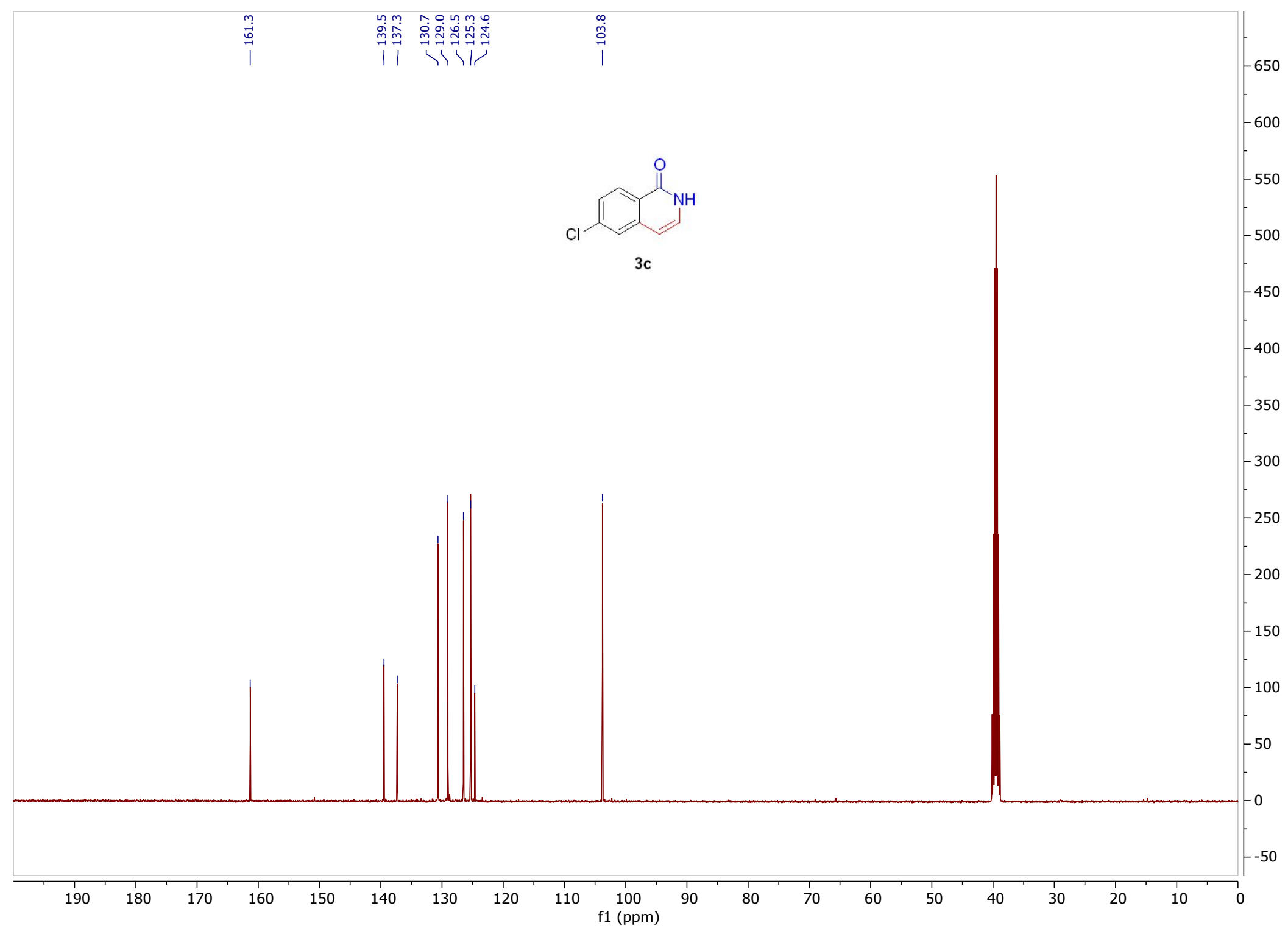


Figure S41. ${ }^{1} \mathrm{H}-\mathrm{NMR}$ (600 MHz, DMSO-d6) for compound 3d.

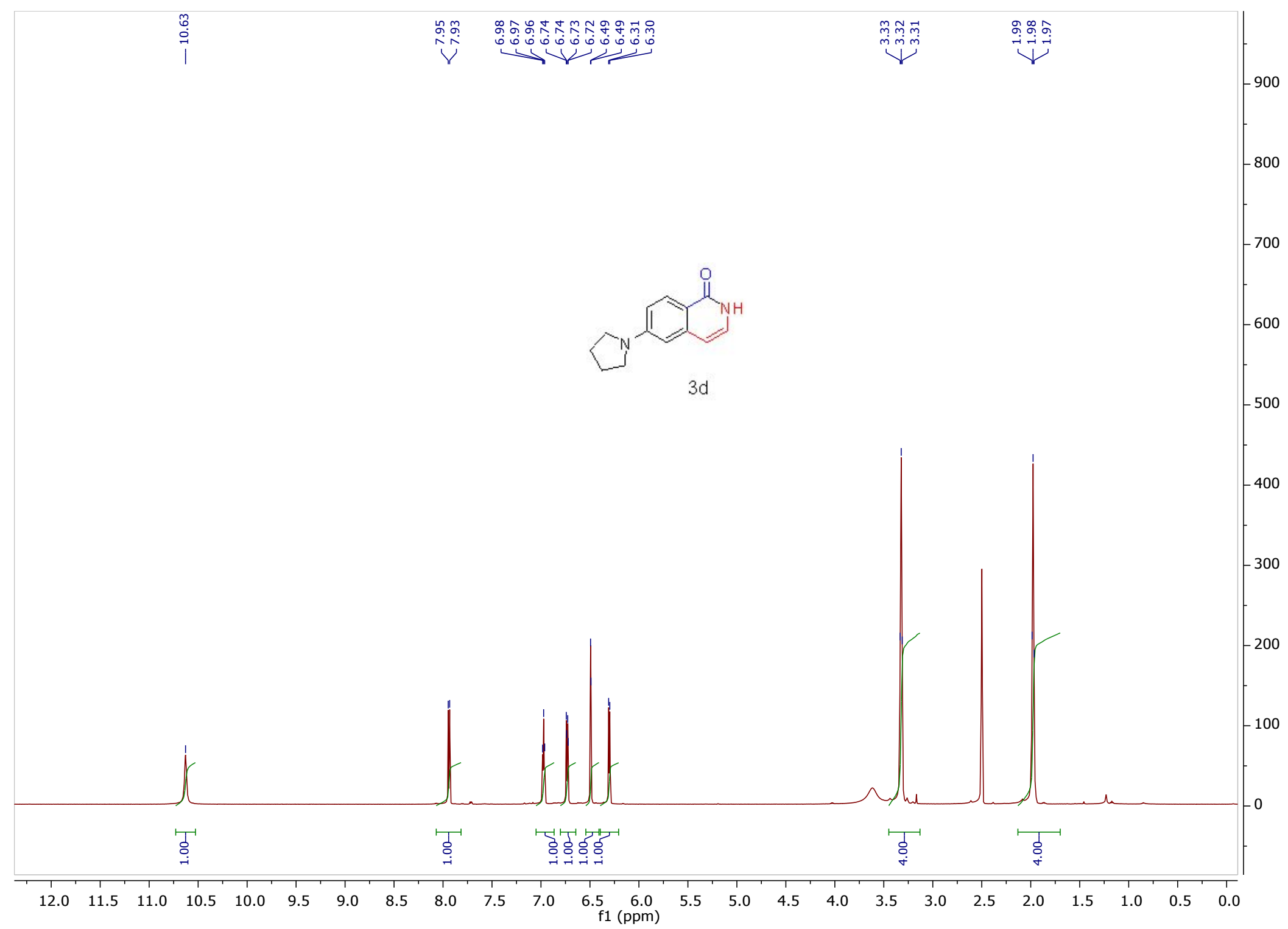


Figure S42. ${ }^{13} \mathrm{C}\left\{{ }^{1} \mathrm{H}\right\}-\mathrm{NMR}$ (151 MHz, DMSO-d6) for compound 3d.

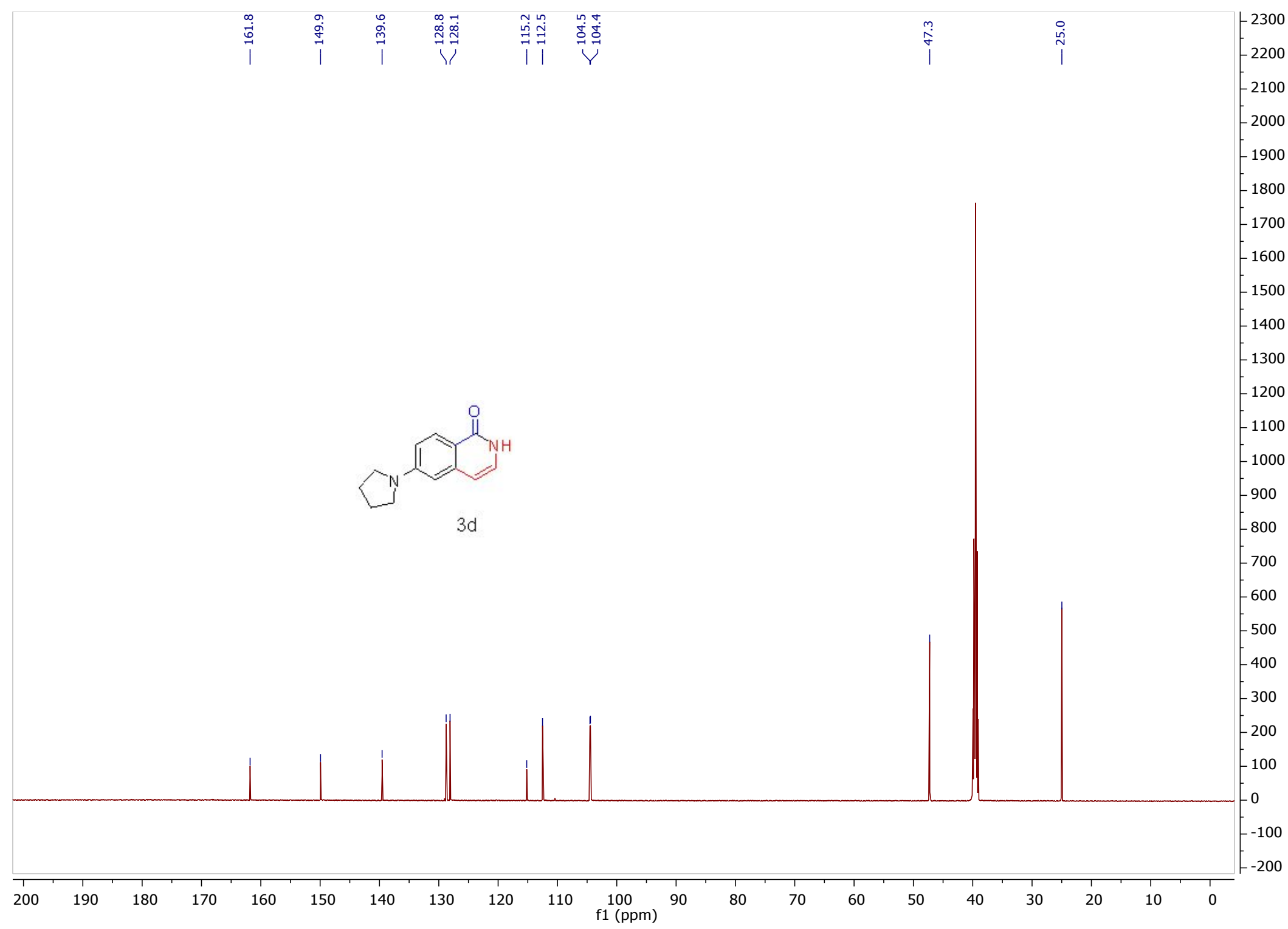


Figure S43. ${ }^{1} \mathrm{H}-\mathrm{NMR}(400 \mathrm{MHz}$, DMSO-d6) for compound $3 \mathrm{e}$.

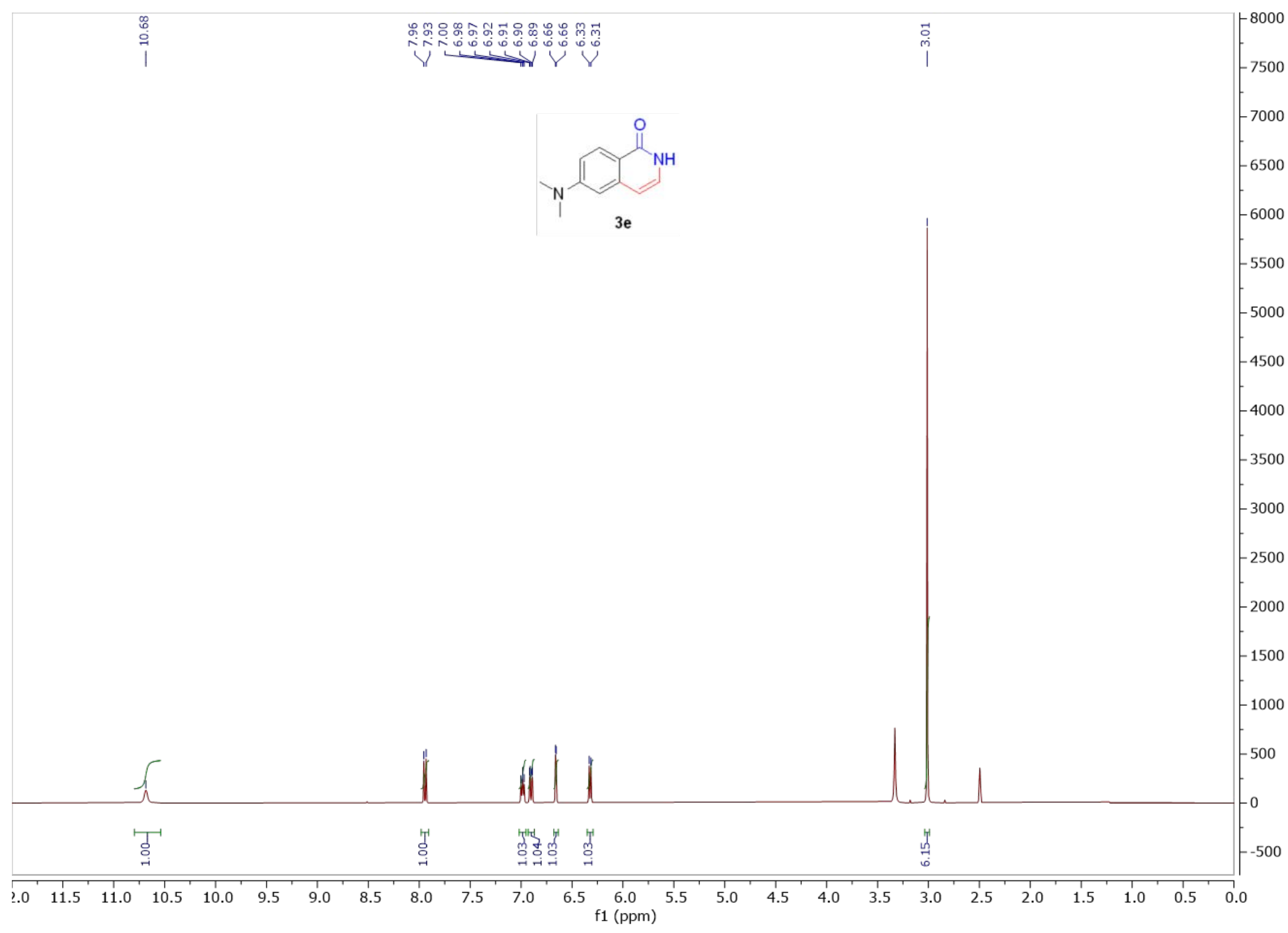


Figure S44. ${ }^{13} \mathrm{C}\left\{{ }^{1} \mathrm{H}\right\}-\mathrm{NMR}$ (101 MHz, DMSO-d6) for compound 3e.

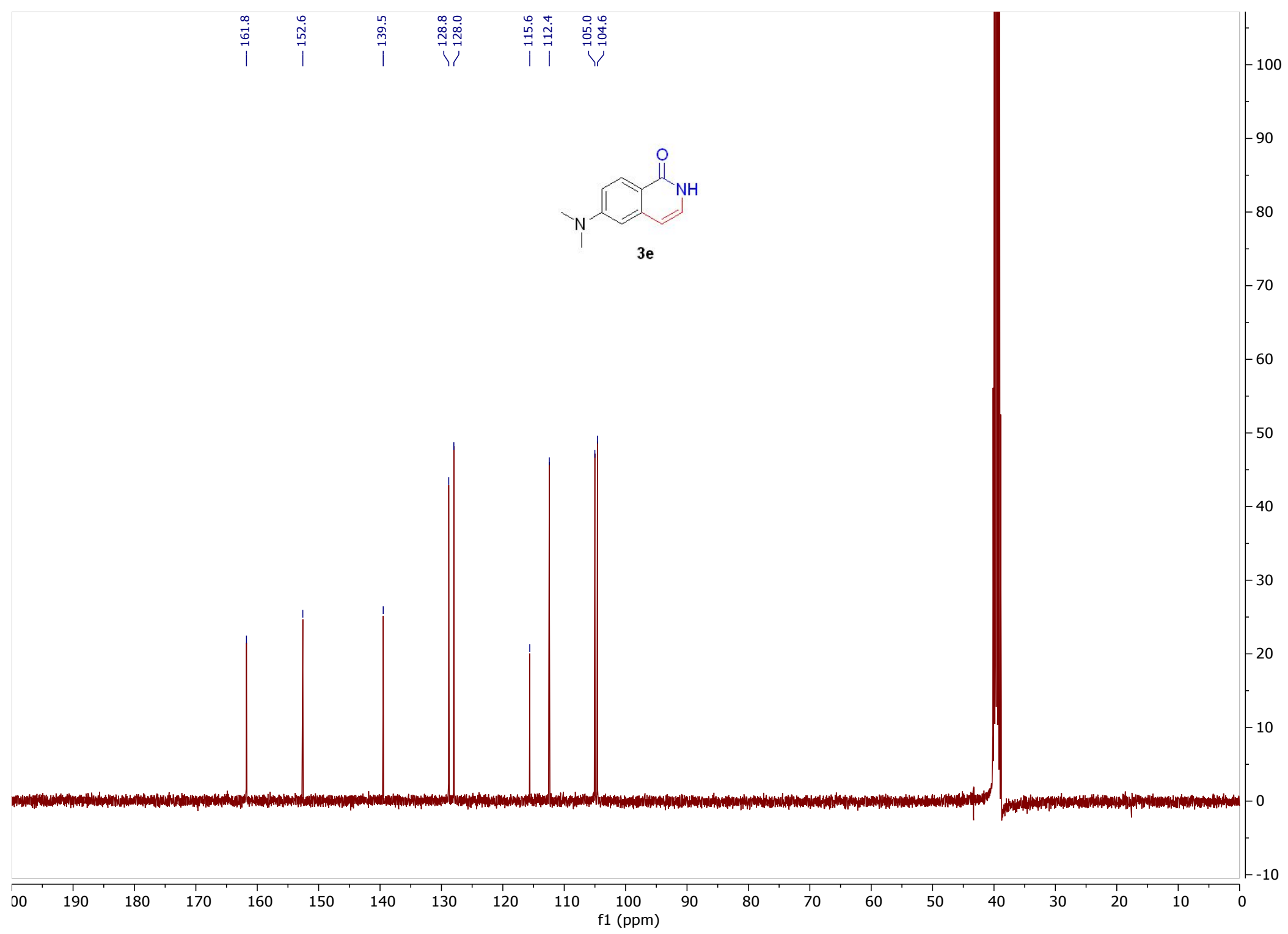


Figure S45. ${ }^{1} \mathrm{H}-\mathrm{NMR}$ (400 MHz, DMSO-d6) for compound $3 \mathrm{f}$.

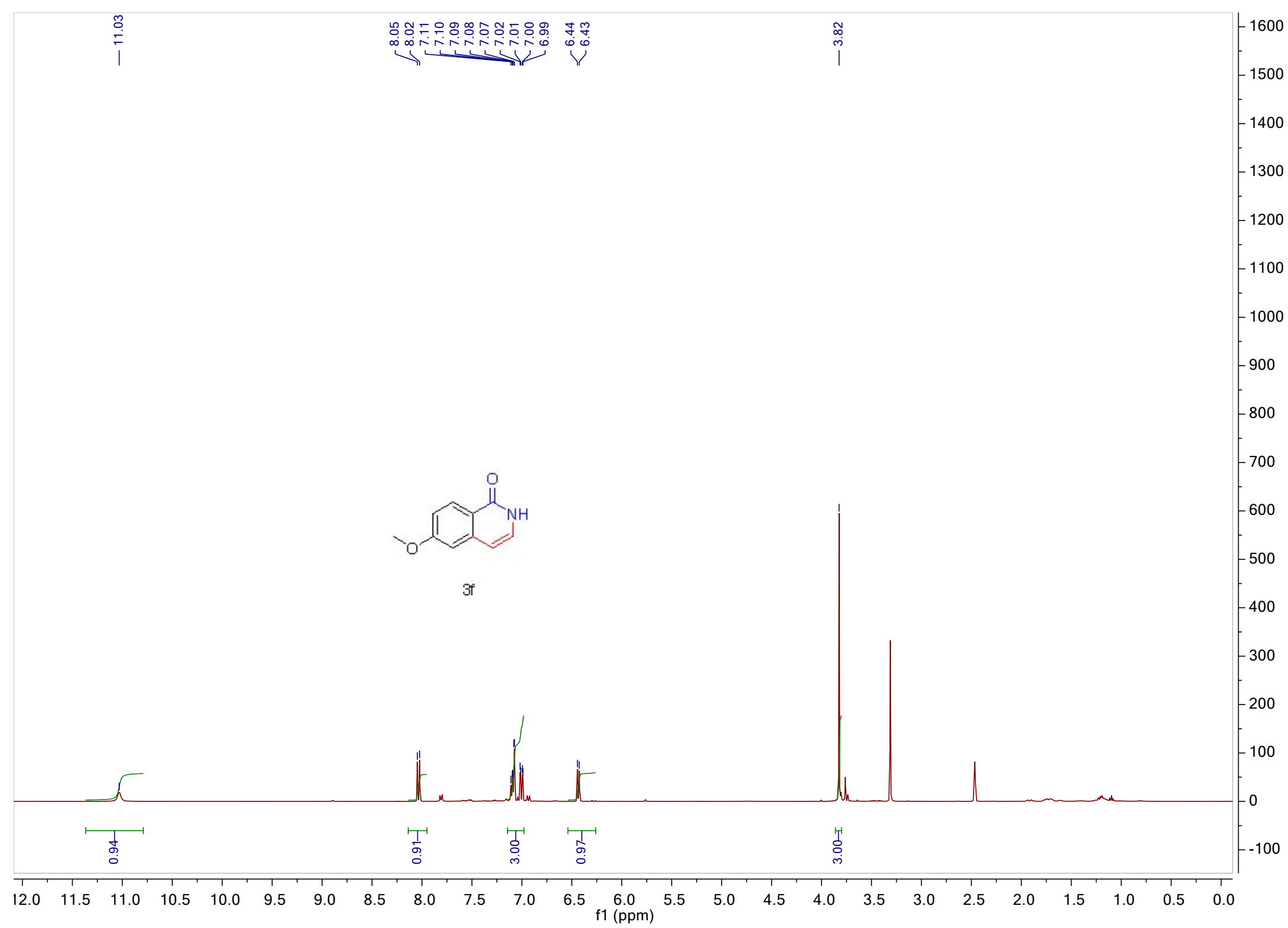


Figure S46. ${ }^{13} \mathrm{C}\left\{{ }^{1} \mathrm{H}\right\}-\mathrm{NMR}$ (101 MHz, DMSO-d6) for compound $3 f$.

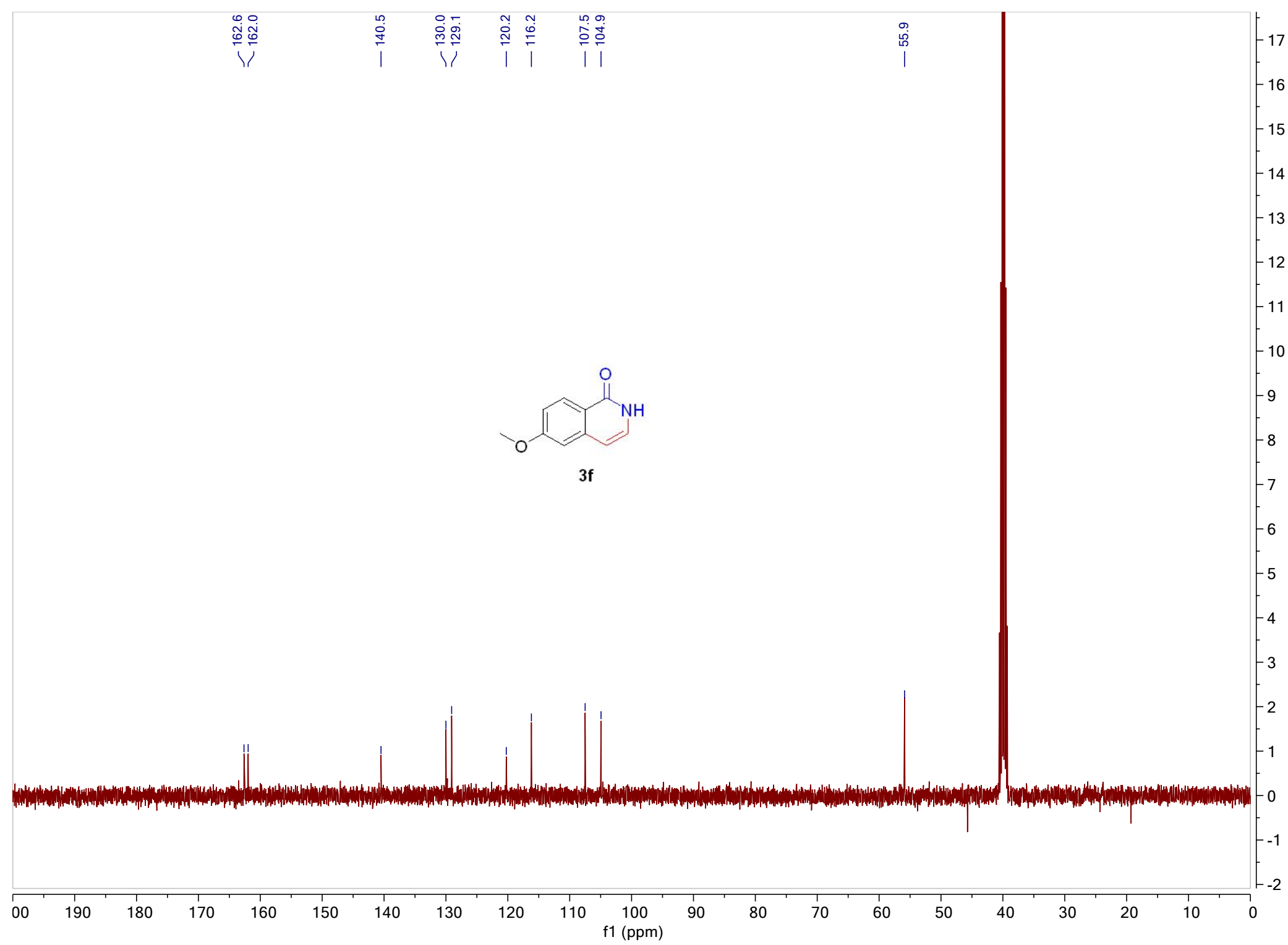


Figure S47. ${ }^{1} \mathrm{H}-\mathrm{NMR}(600 \mathrm{MHz}$, DMSO-d6) for compound 3g.

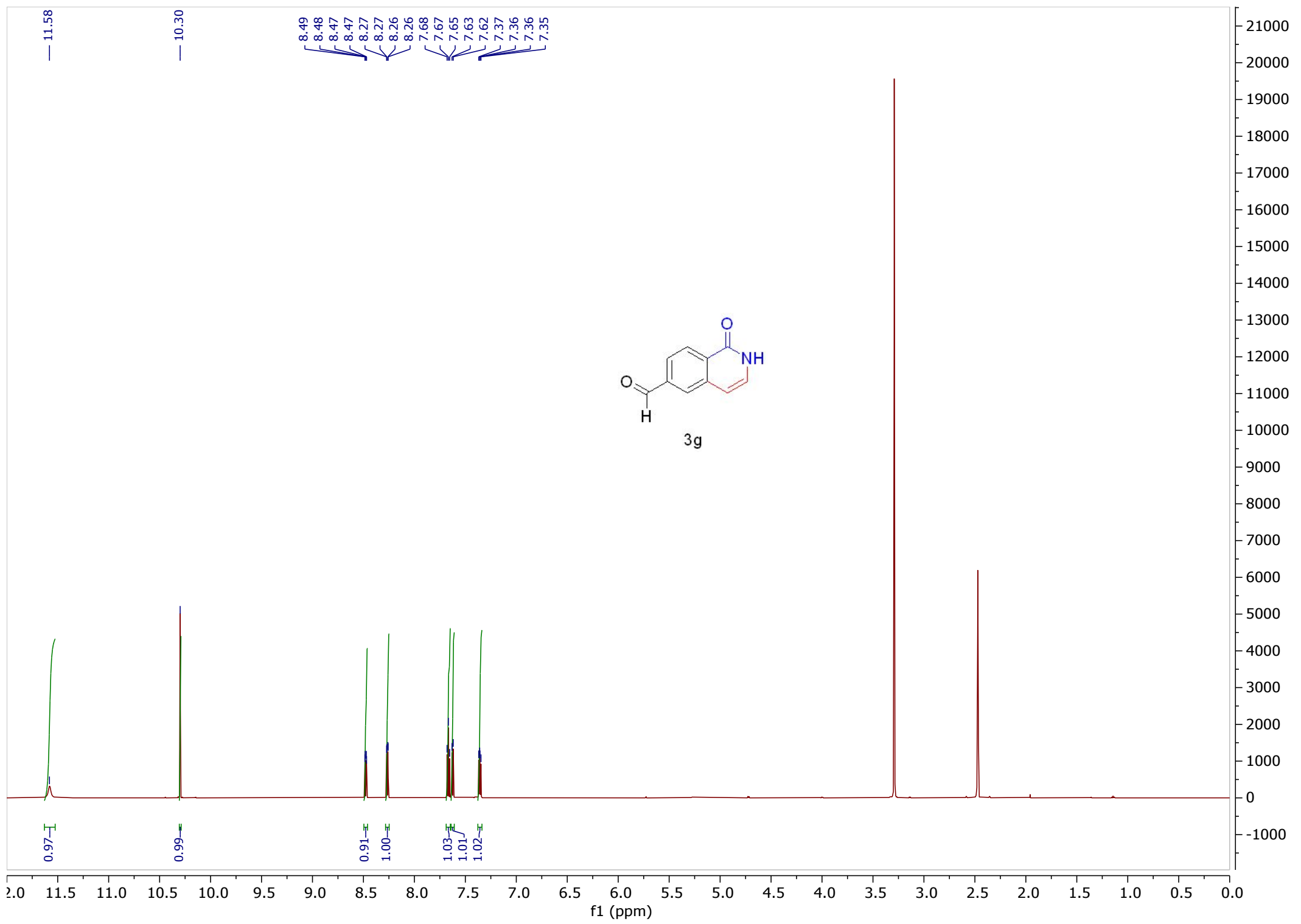

SI-48 
Figure S48. ${ }^{13} \mathrm{C}\left\{{ }^{1} \mathrm{H}\right\}-\mathrm{NMR}$ (151 MHz, DMSO-d6) for compound 3g.

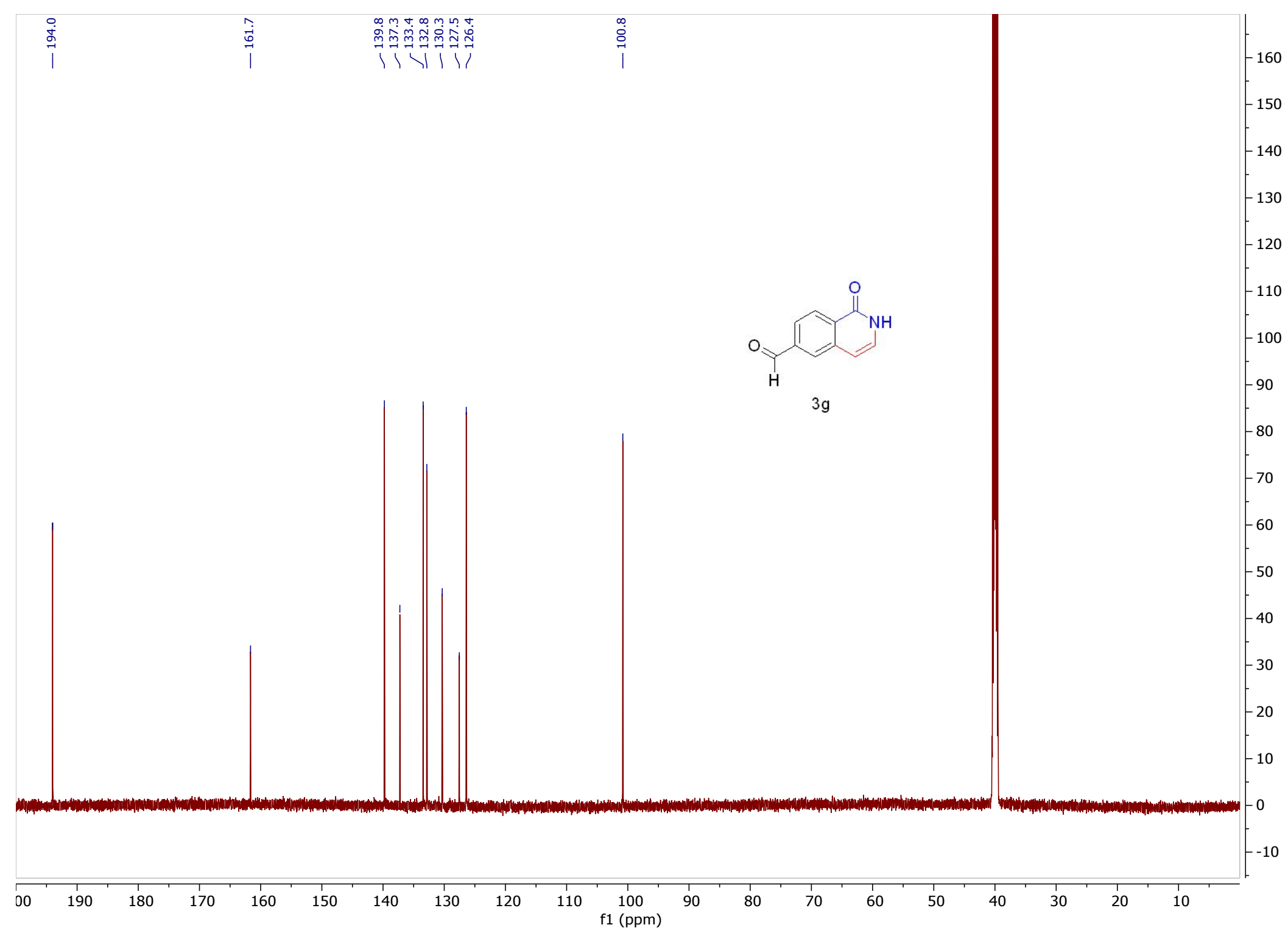


Figure S49. ${ }^{1} \mathrm{H}-\mathrm{NMR}$ (400 MHz, DMSO-d6) for compound $3 \mathrm{~h}$.

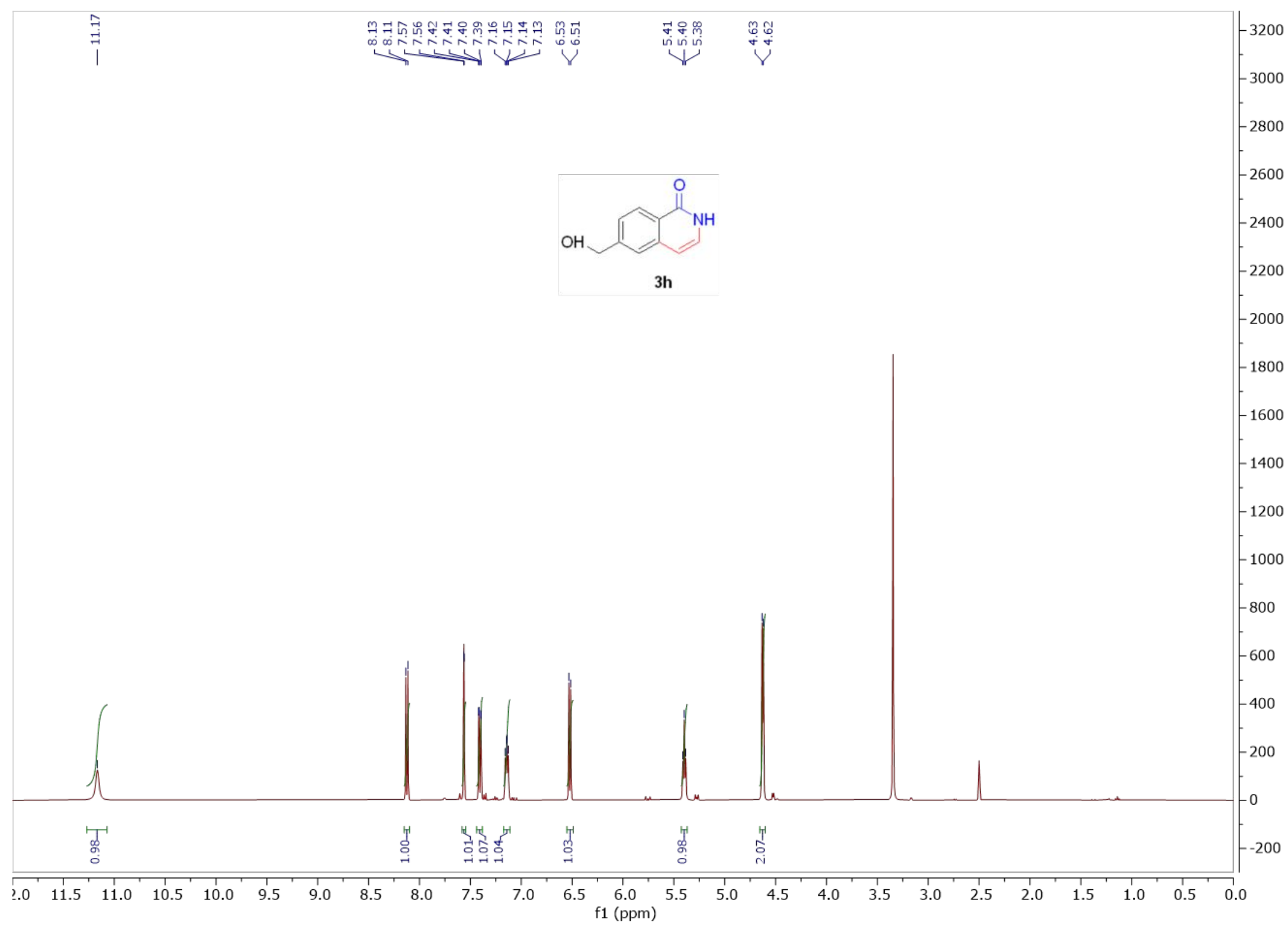


Figure S50. ${ }^{13} \mathrm{C}\left\{{ }^{1} \mathrm{H}\right\}-\mathrm{NMR}$ (101 MHz, DMSO-d6) for compound $3 \mathrm{~h}$.

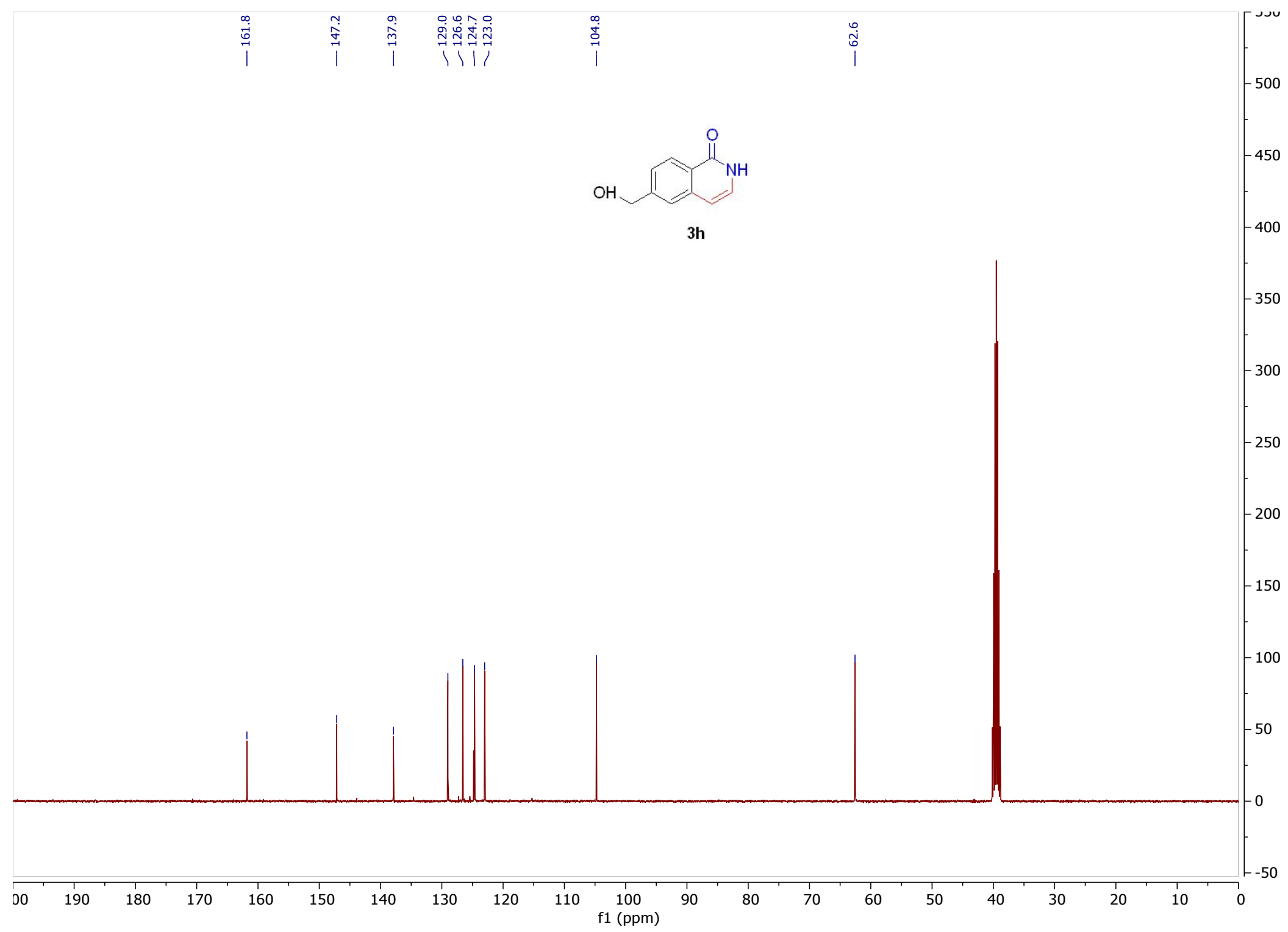


Figure S51. ${ }^{1} \mathrm{H}-\mathrm{NMR}$ (400 MHz, DMSO-d6) for compound 3i

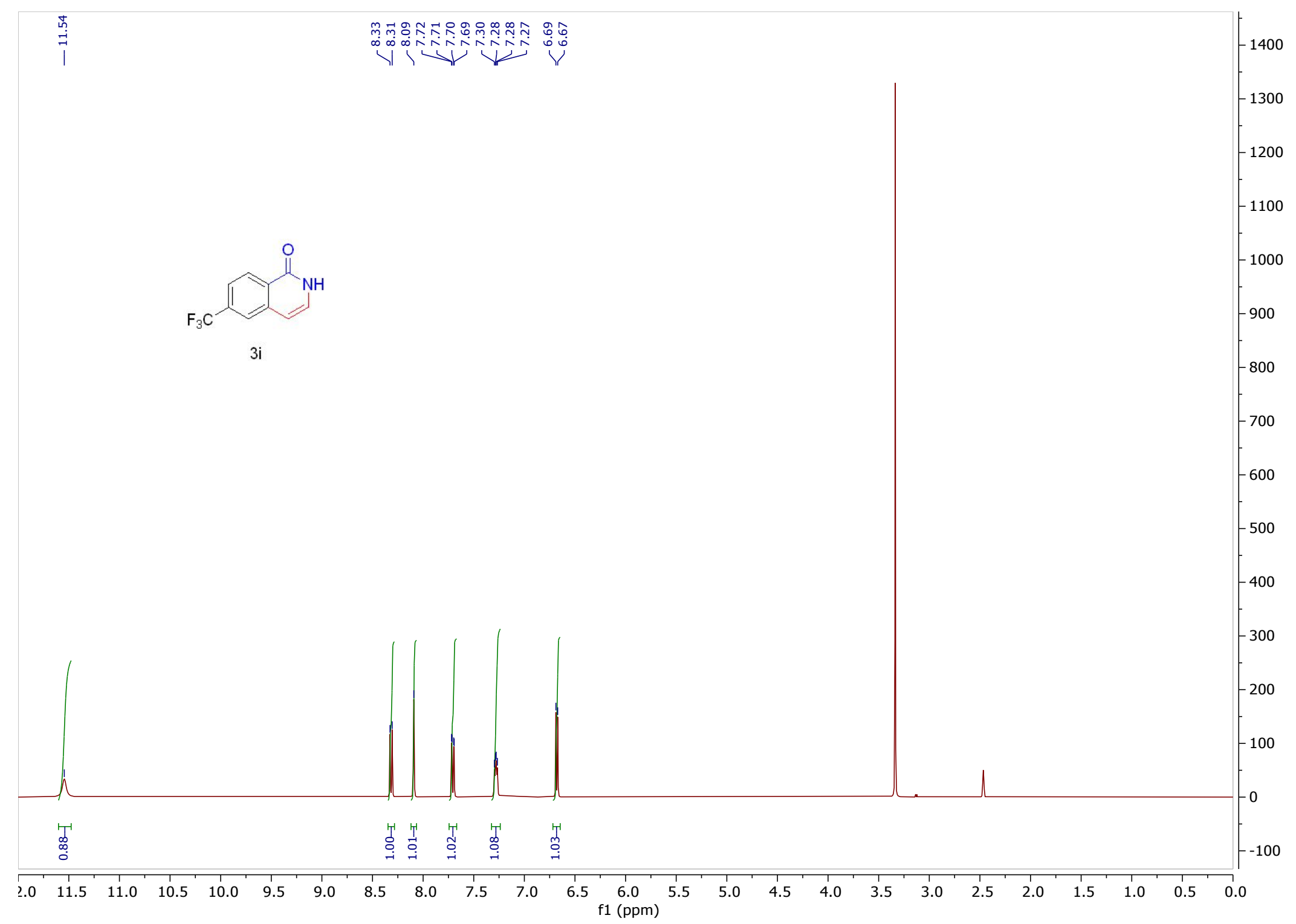


Figure S52. ${ }^{13} \mathrm{C}\left\{{ }^{1} \mathrm{H}\right\}-\mathrm{NMR}$ (101 MHz, DMSO-d6) for compound 3i.

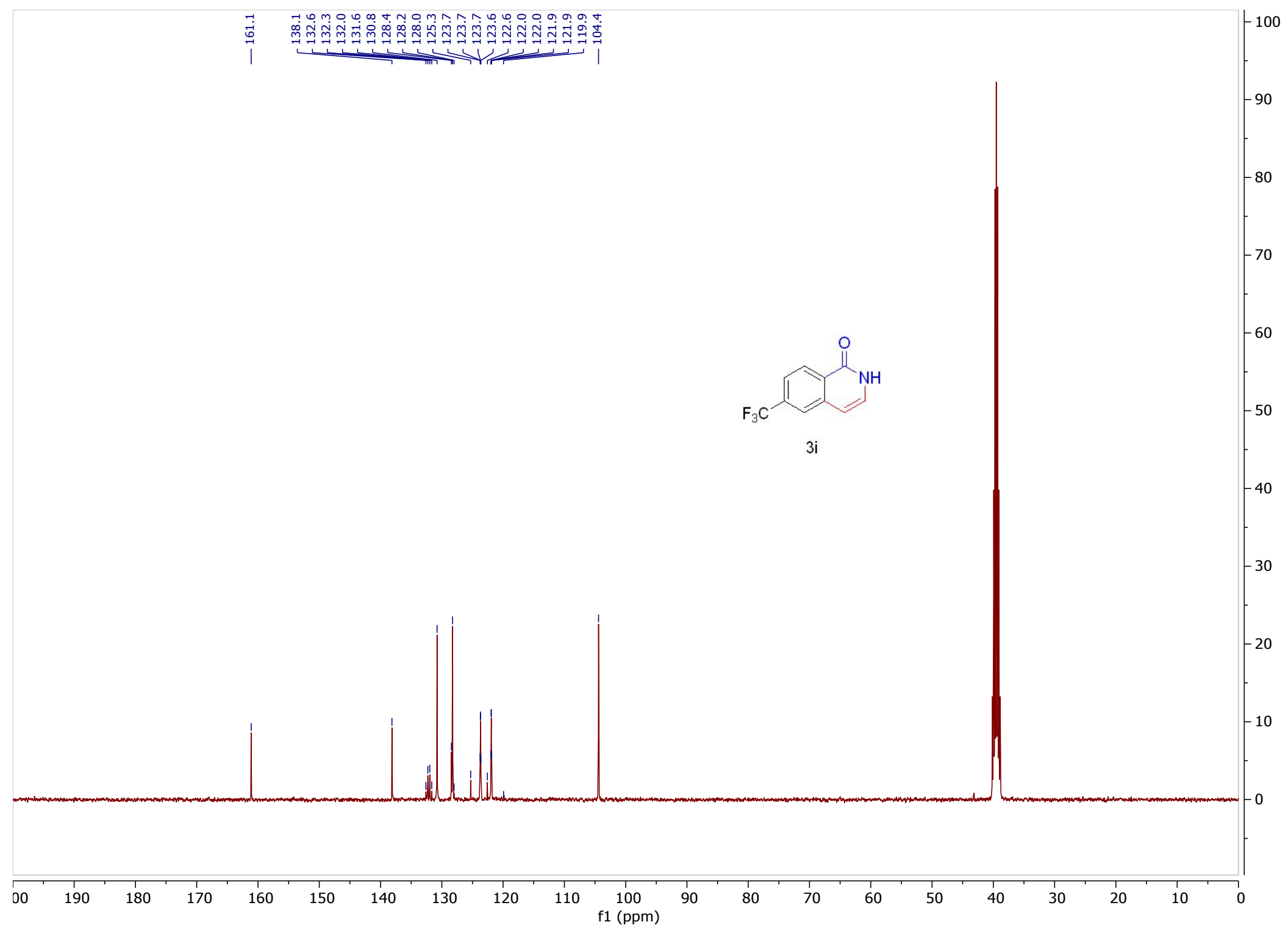


Figure S53. ${ }^{19} \mathrm{~F}-\mathrm{NMR}$ (376 MHz, DMSO-d6) for compound 3i.

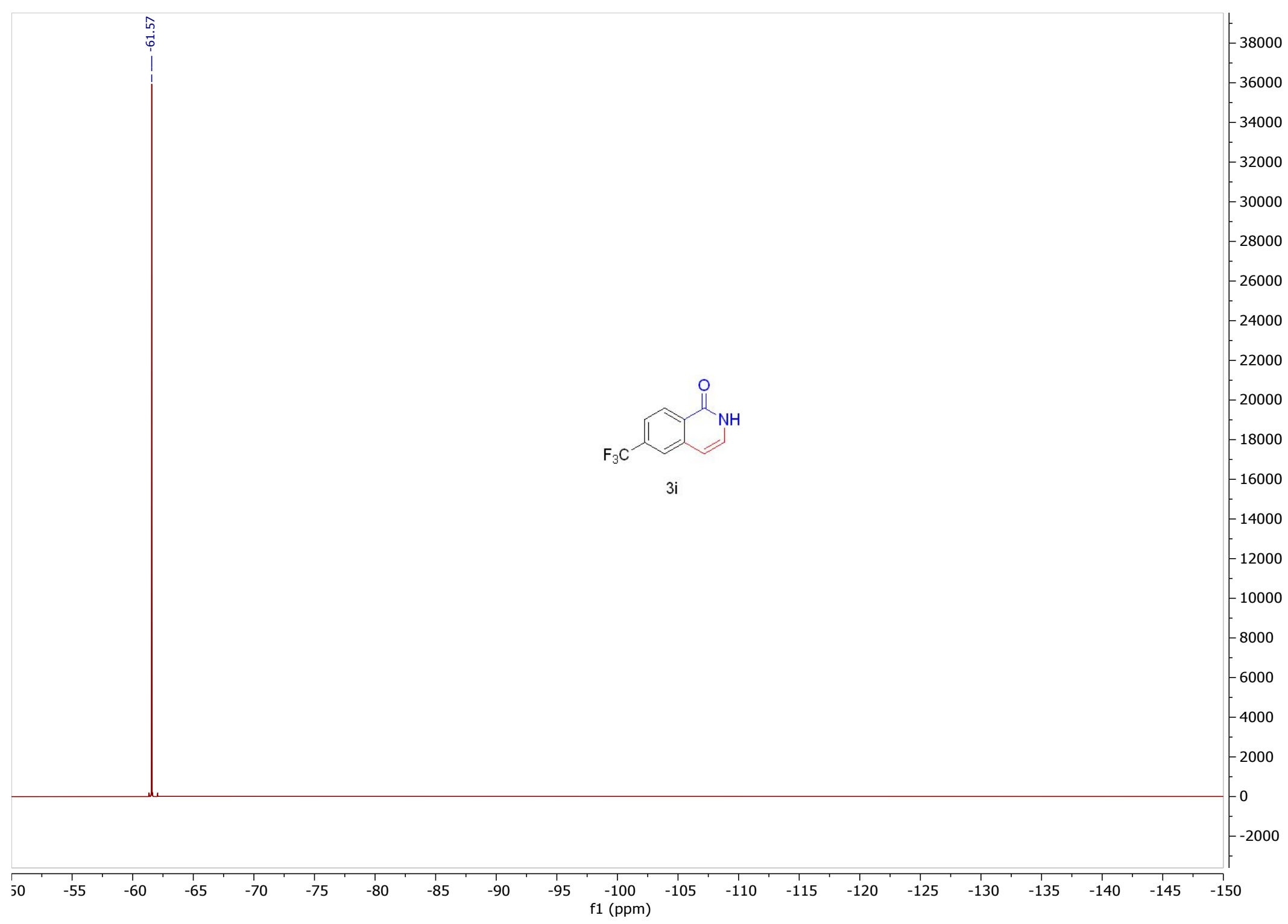


Figure S54. ${ }^{1} \mathrm{H}-\mathrm{NMR} f(400 \mathrm{MHz}$, DMSO-d6) or compound $3 \mathrm{j}$

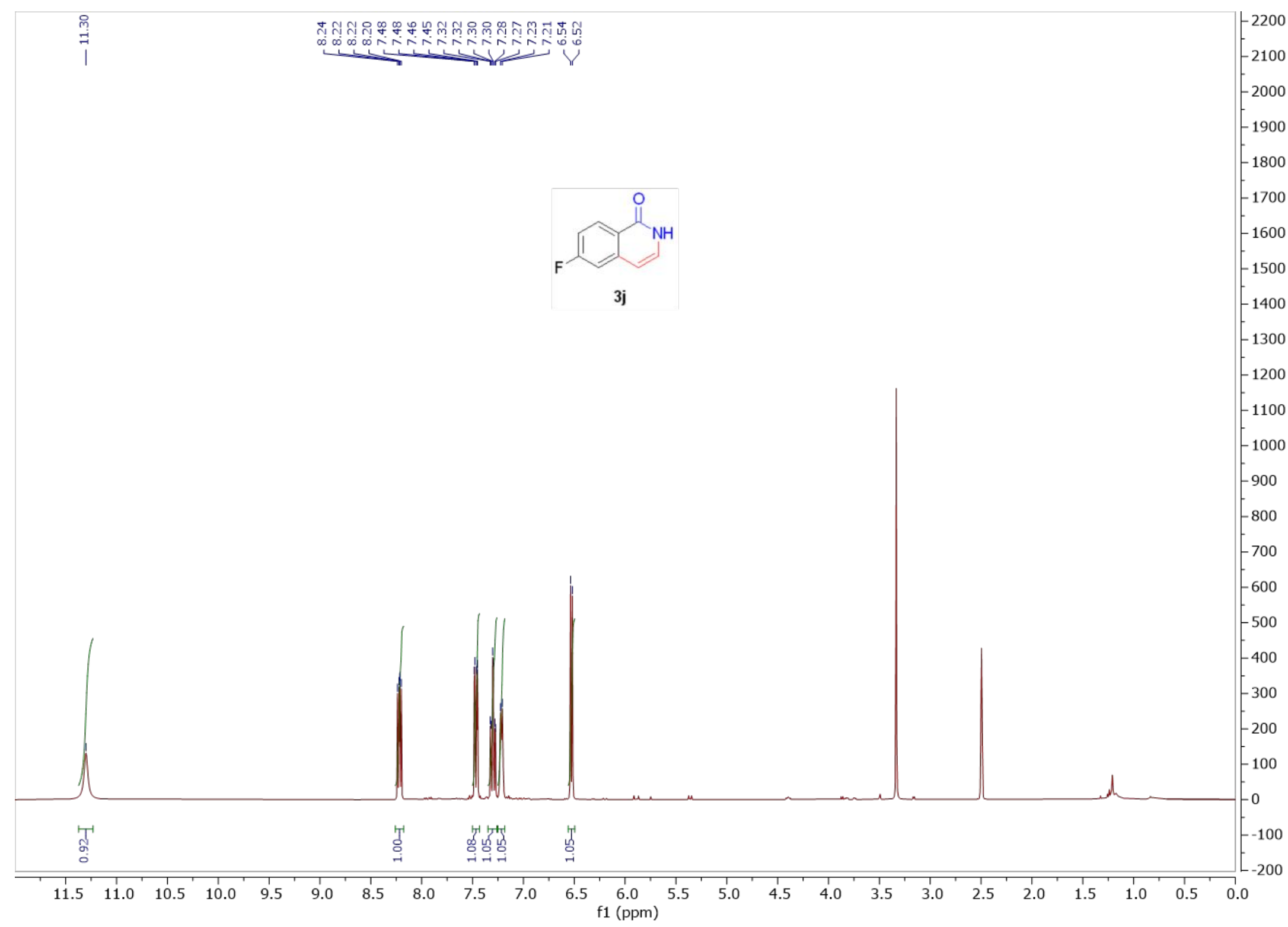


Figure S55. ${ }^{13} \mathrm{C}\left\{{ }^{1} \mathrm{H}\right\}-\mathrm{NMR}$ (101 MHz, DMSO-d6) for compound 3j.

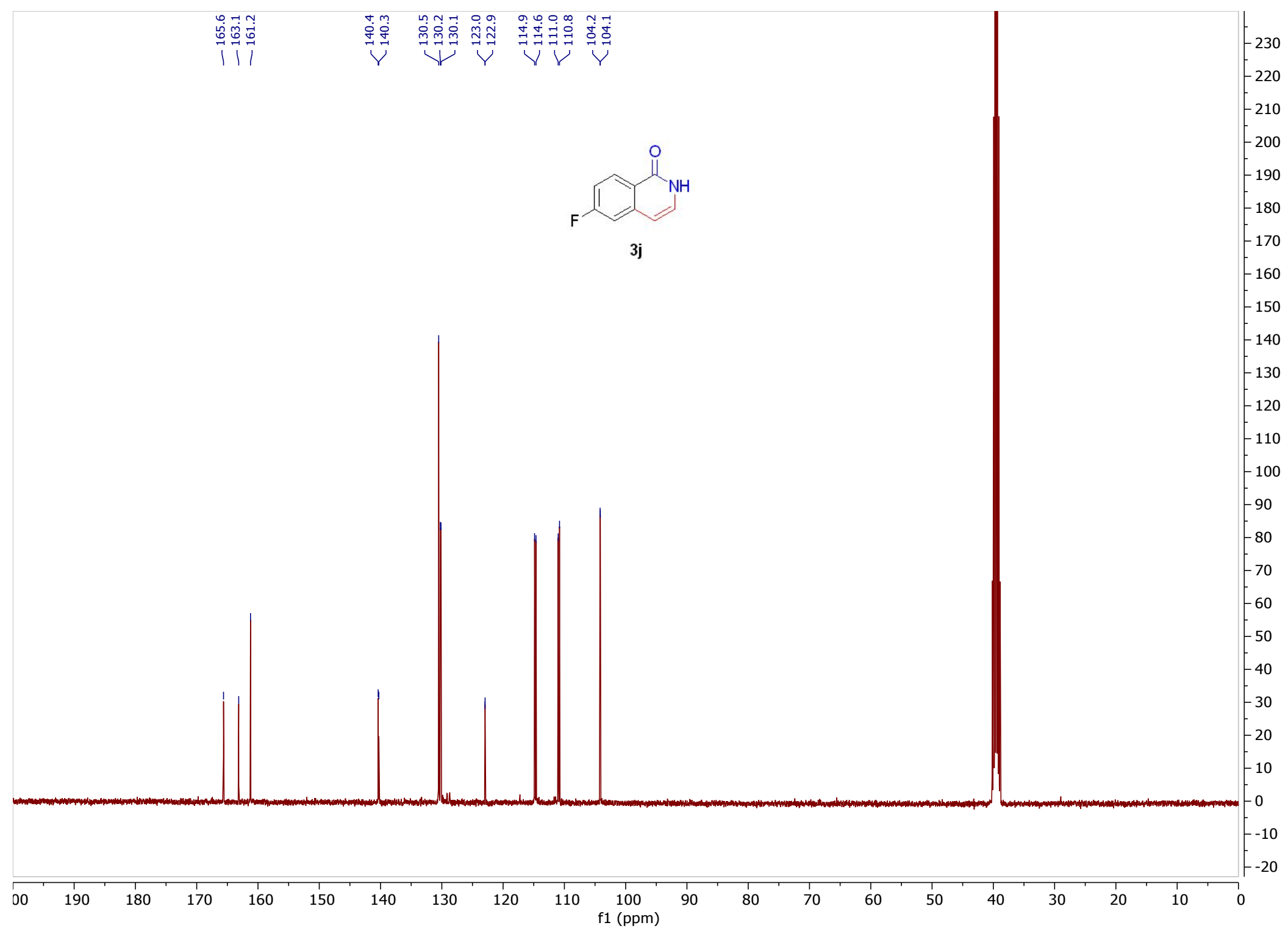


Figure S56. ${ }^{19} \mathrm{~F}-\mathrm{NMR}$ (471 MHz, DMSO-d6) for compound 3j.

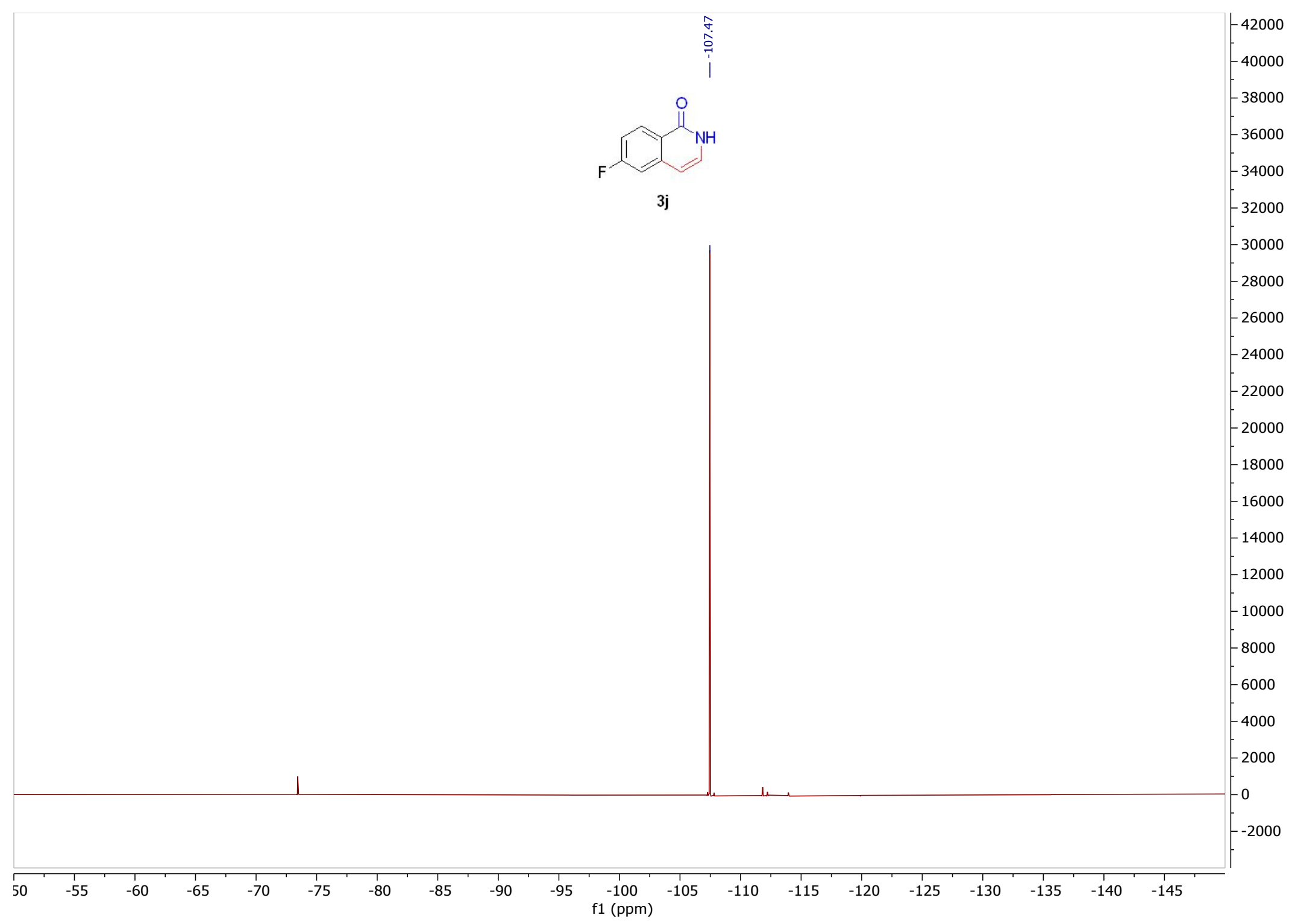


Figure S57. ${ }^{1} \mathrm{H}-\mathrm{NMR}$ (400 MHz, DMSO-d6) for compound 3k.

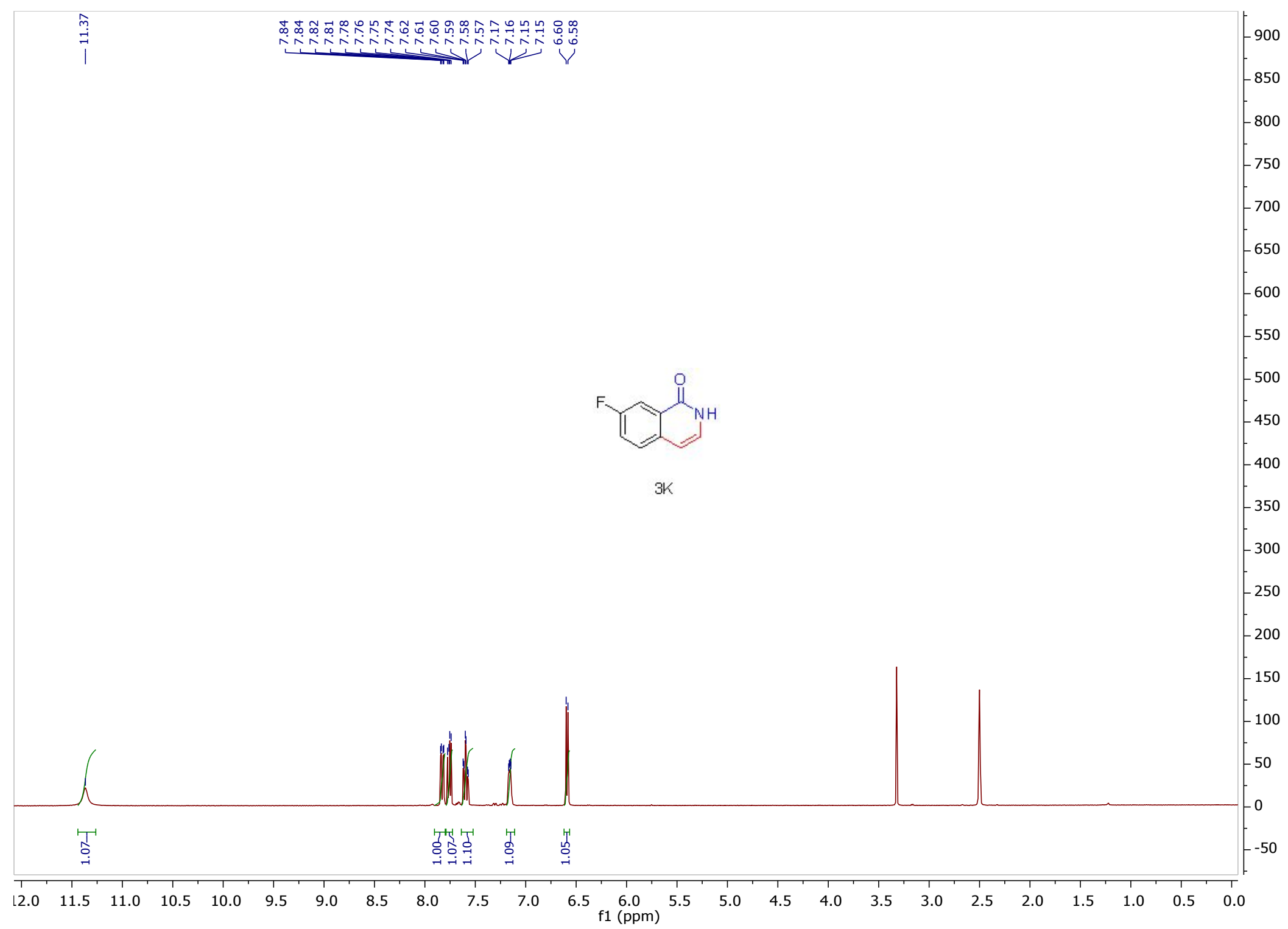


Figure S58. ${ }^{13} \mathrm{C}\left\{{ }^{1} \mathrm{H}\right\}-\mathrm{NMR}$ (126 MHz, DMSO-d6) for compound 3k.

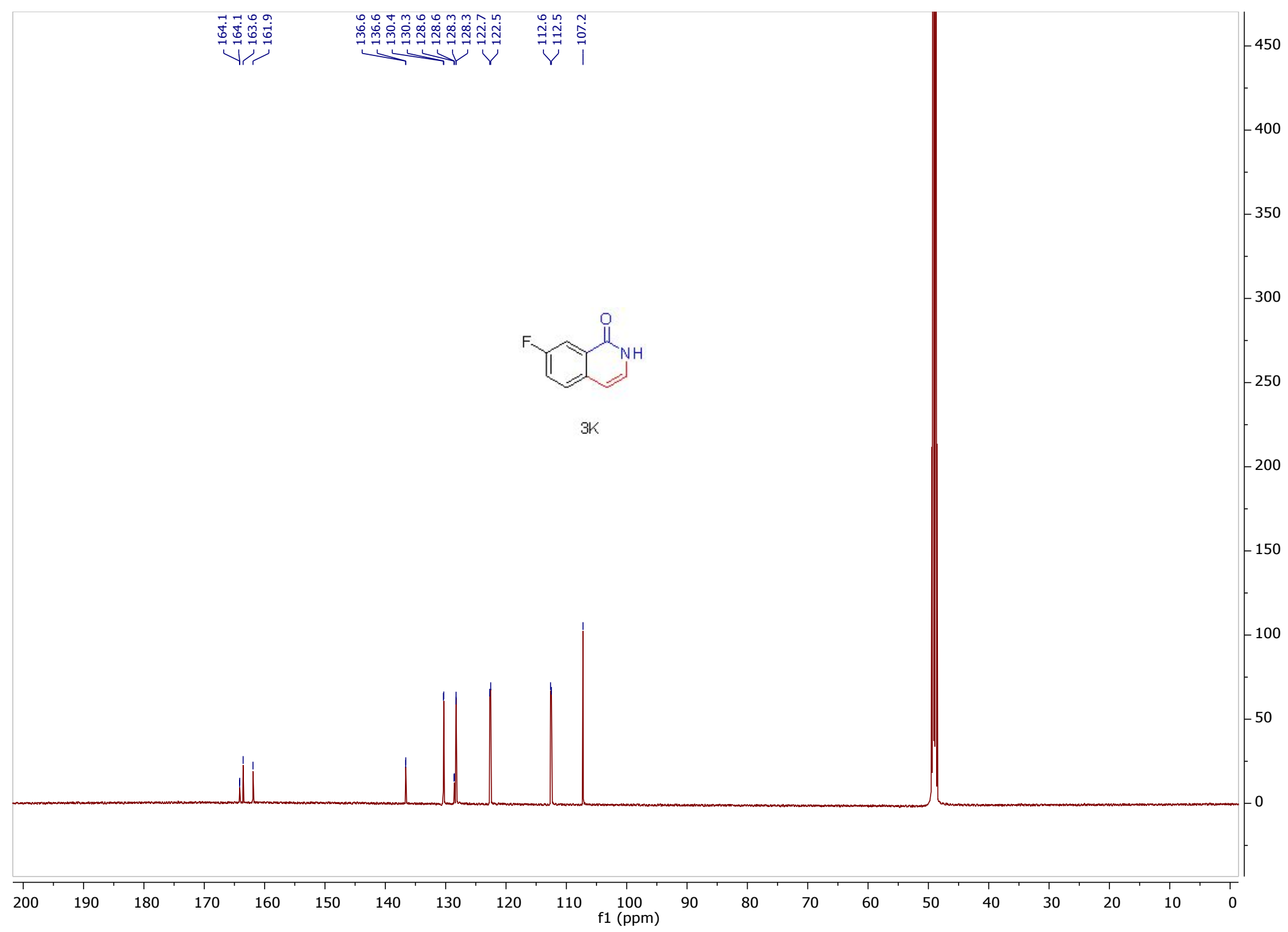


Figure S59. ${ }^{19} \mathrm{~F}-\mathrm{NMR}$ (376 MHz, DMSO-d6) for compound 3k.

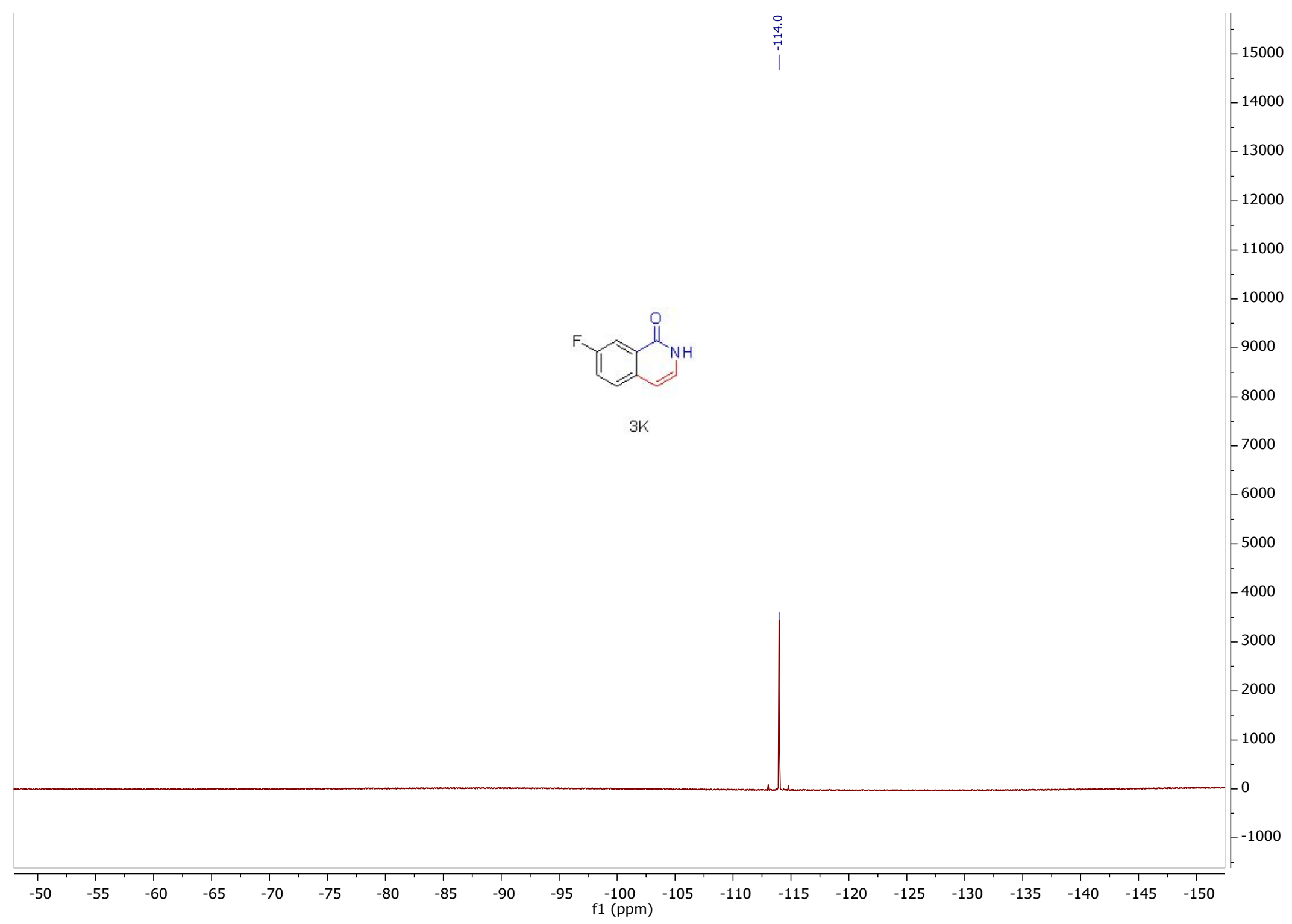


Figure S60. ${ }^{1} \mathrm{H}-\mathrm{NMR}$ (400 MHz, DMSO-d6) for compound 31.

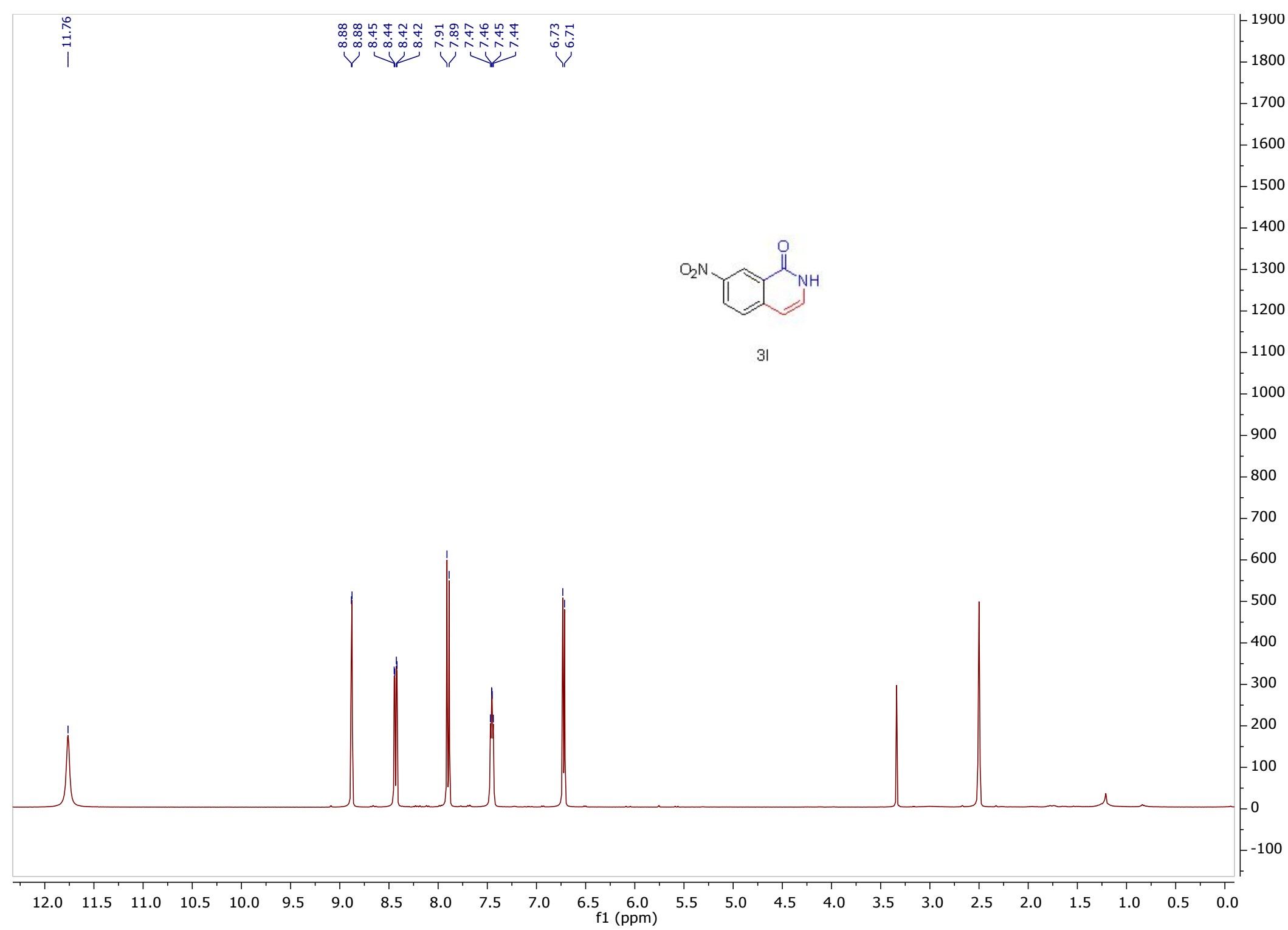


Figure S61. ${ }^{13} \mathrm{C}\left\{{ }^{1} \mathrm{H}\right\}-\mathrm{NMR}$ (151 MHz, DMSO-d6) for compound $3 \mathrm{I}$.

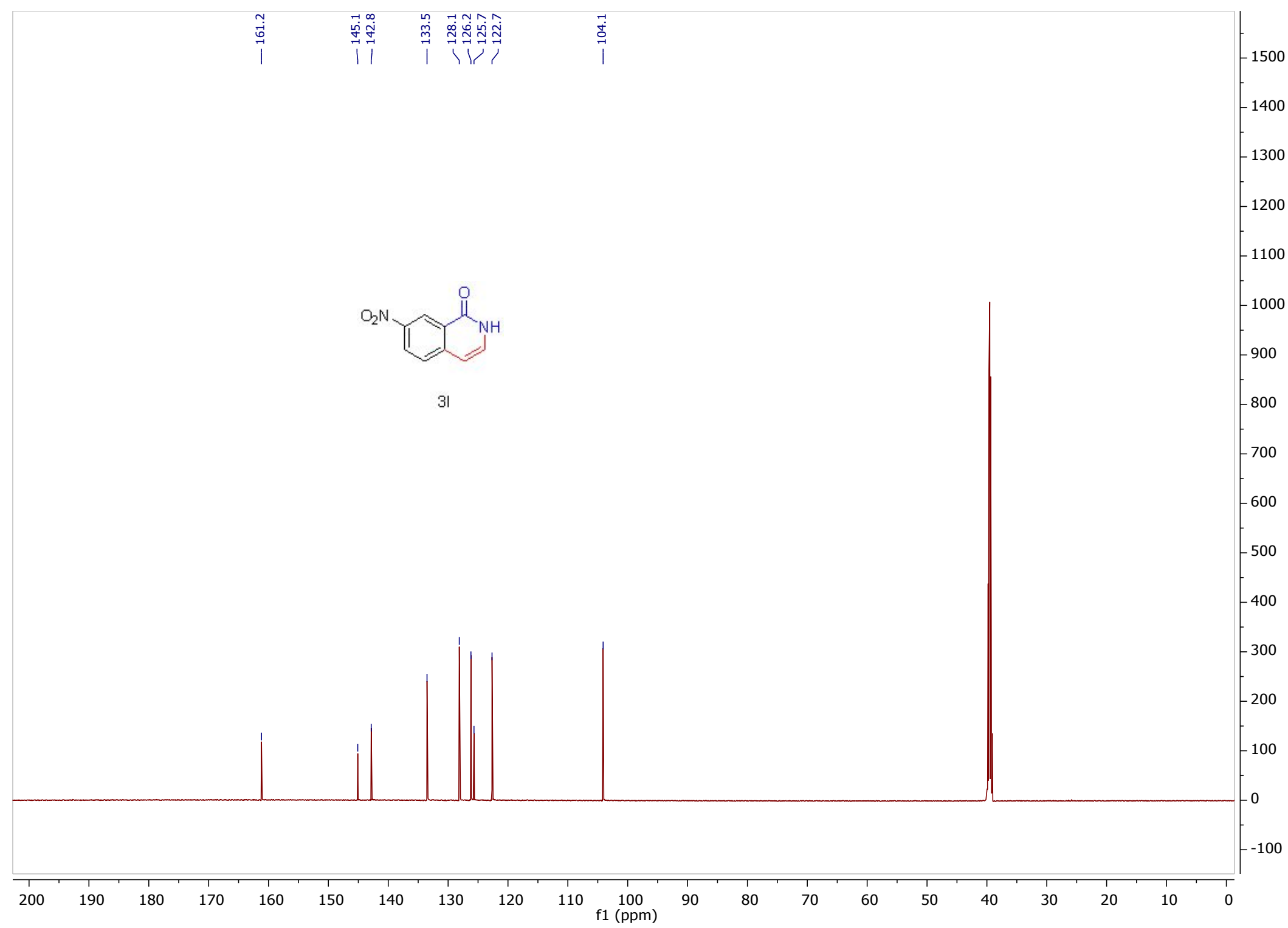


Figure S62. ${ }^{1} \mathrm{H}-\mathrm{NMR}$ (400 MHz, DMSO-d6) for compound $3 \mathrm{~m}$.

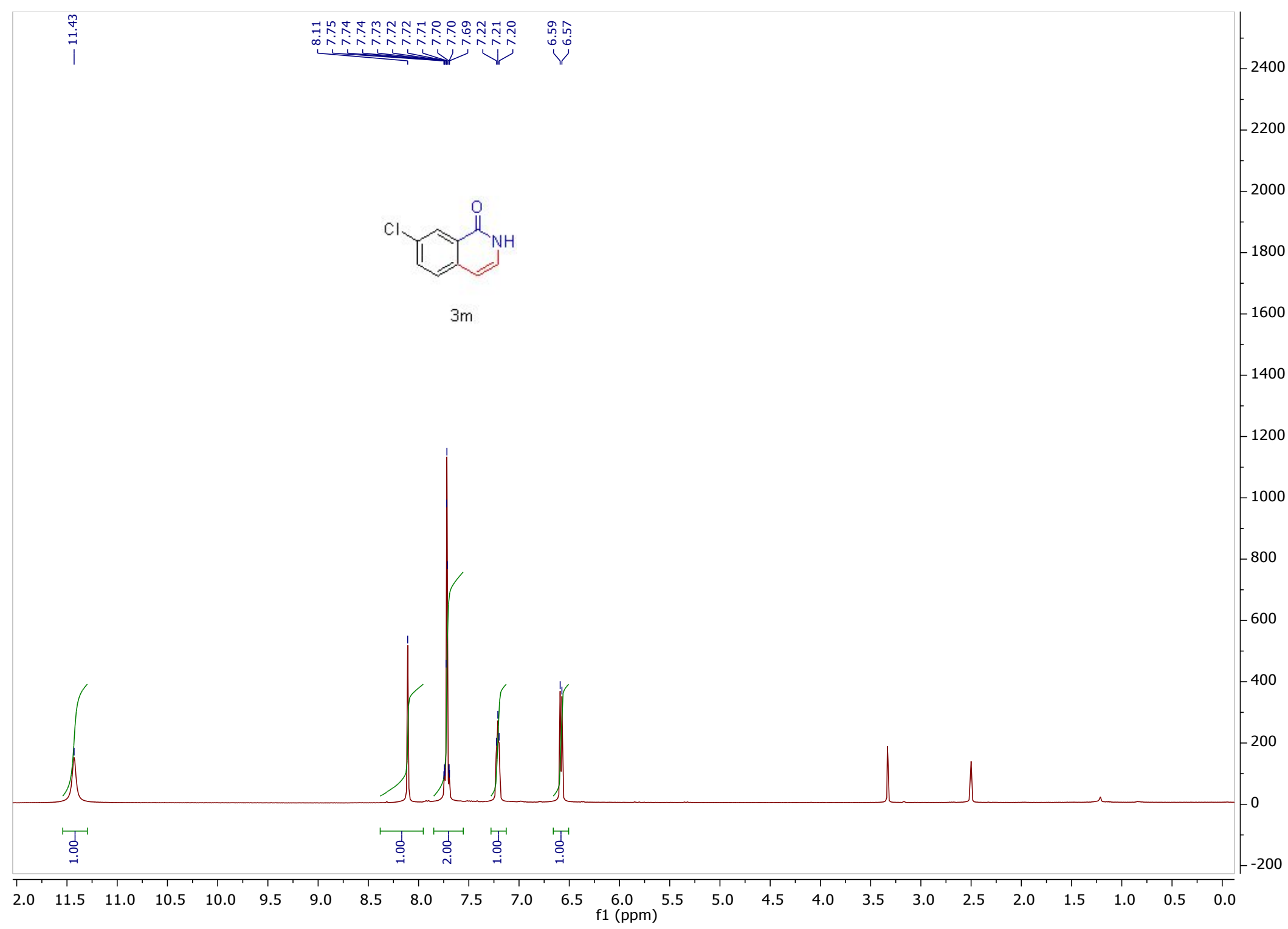


Figure S63. ${ }^{13} \mathrm{C}\left\{{ }^{1} \mathrm{H}\right\}-\mathrm{NMR}$ (101 MHz, DMSO-d6) for compound 3m.

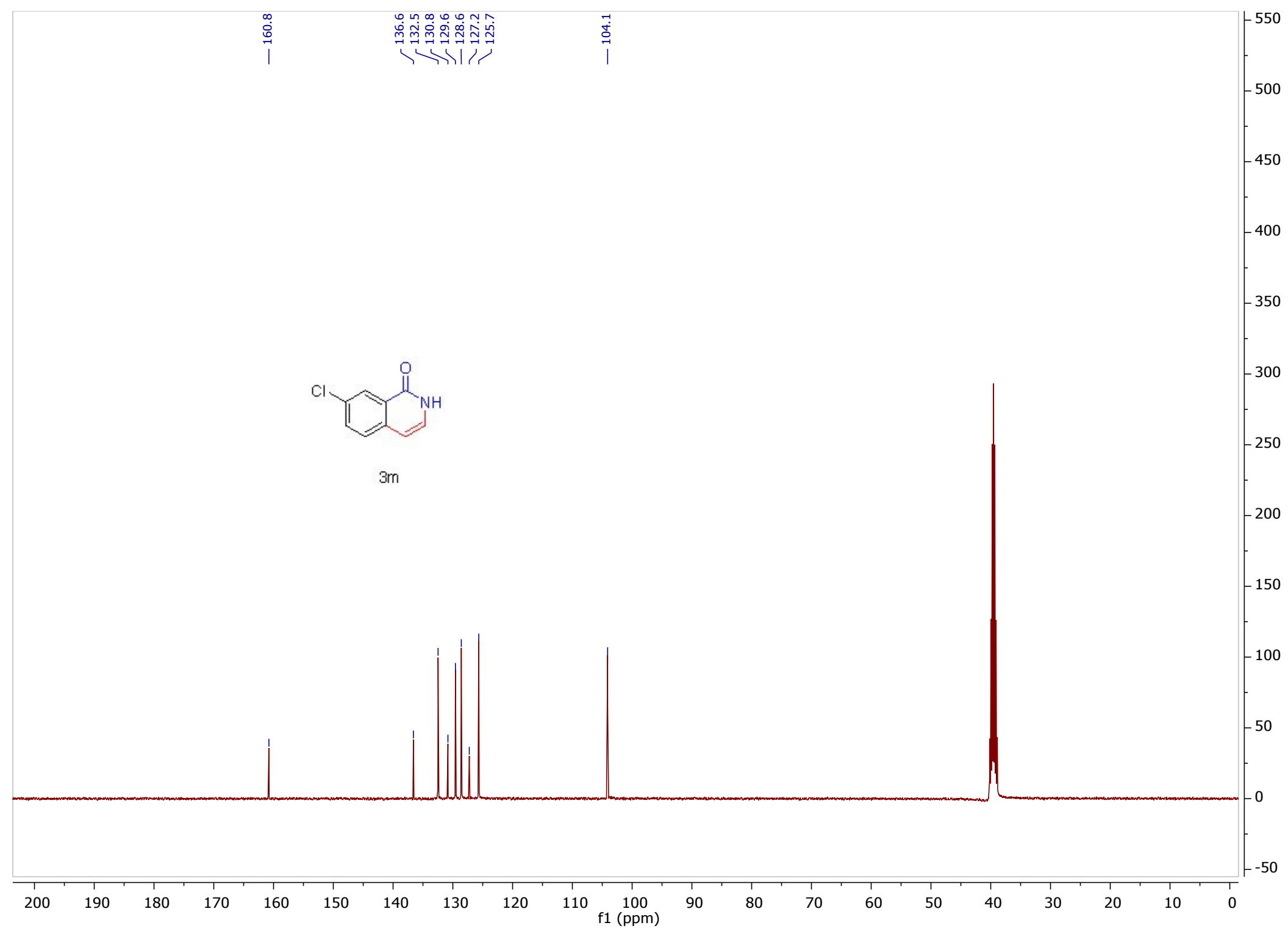


Figure S64. ${ }^{1} \mathrm{H}-\mathrm{NMR}(600 \mathrm{MHz}$, DMSO-d6) for compound $3 \mathrm{n}$.

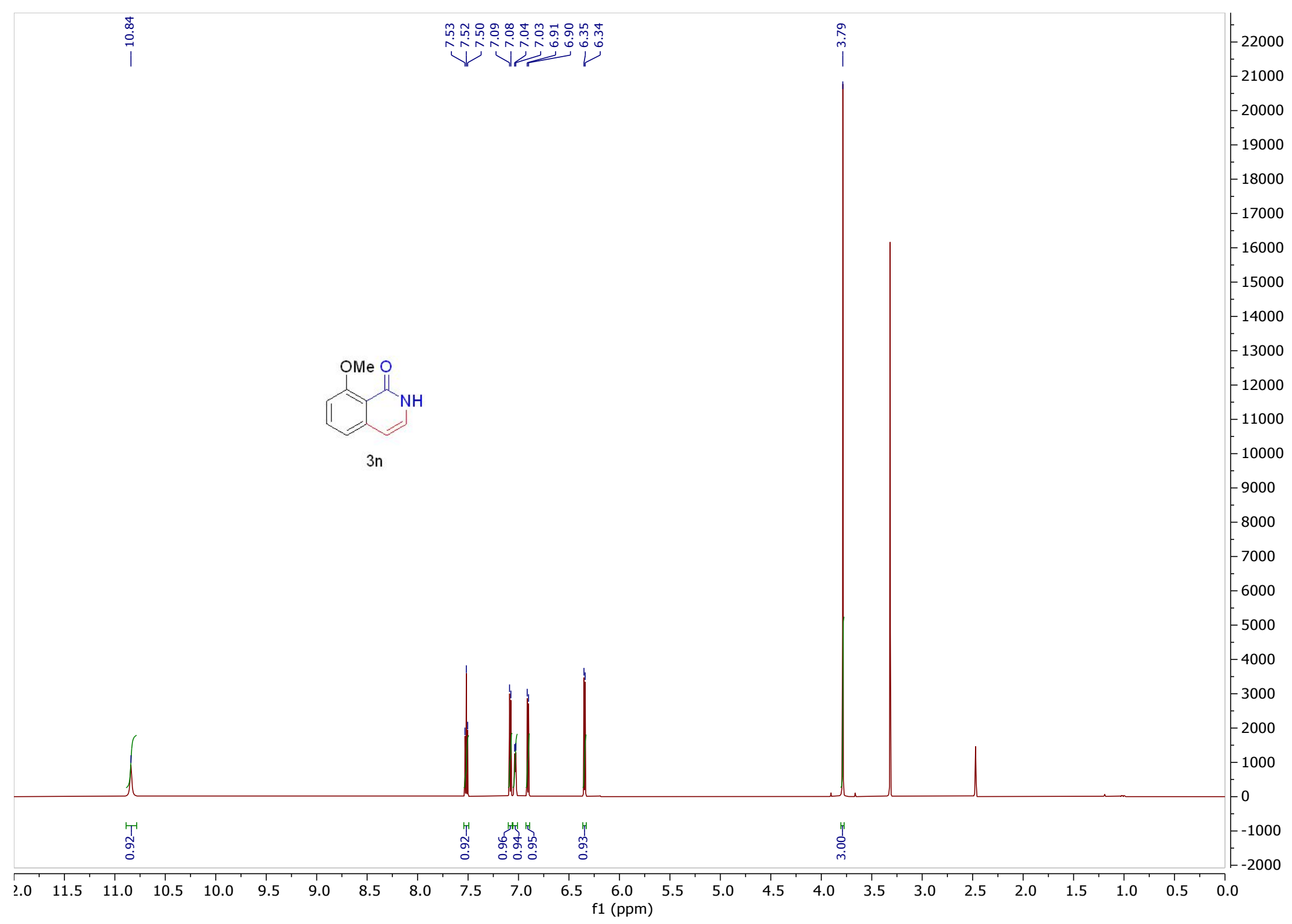


Figure S65. ${ }^{13} \mathrm{C}\left\{{ }^{1} \mathrm{H}\right\}-\mathrm{NMR}$ (151 MHz, DMSO-d6) for compound 3n.

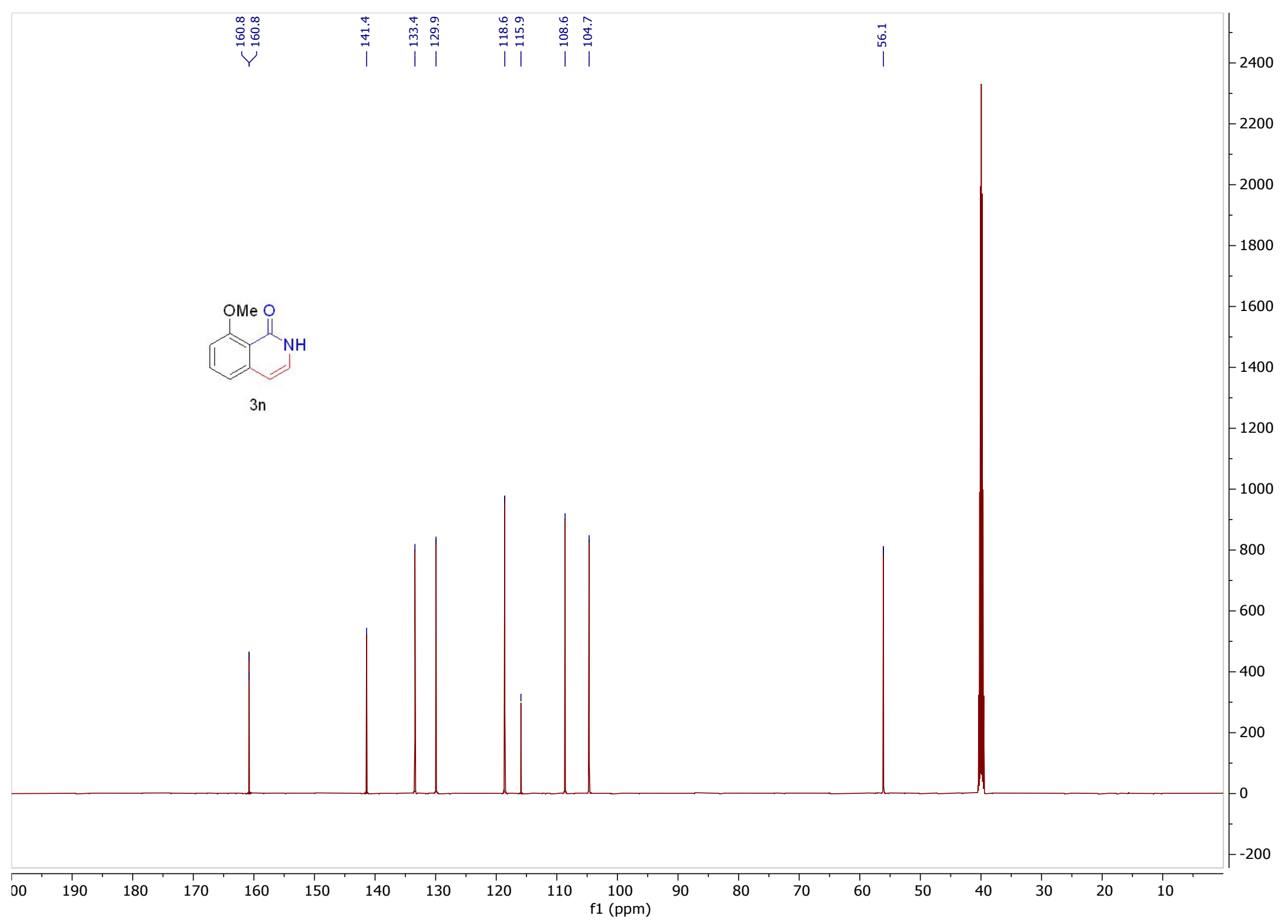


Figure S66. ${ }^{1} \mathrm{H}-\mathrm{NMR}$ (400 MHz, DMSO-d6) for compound 30.

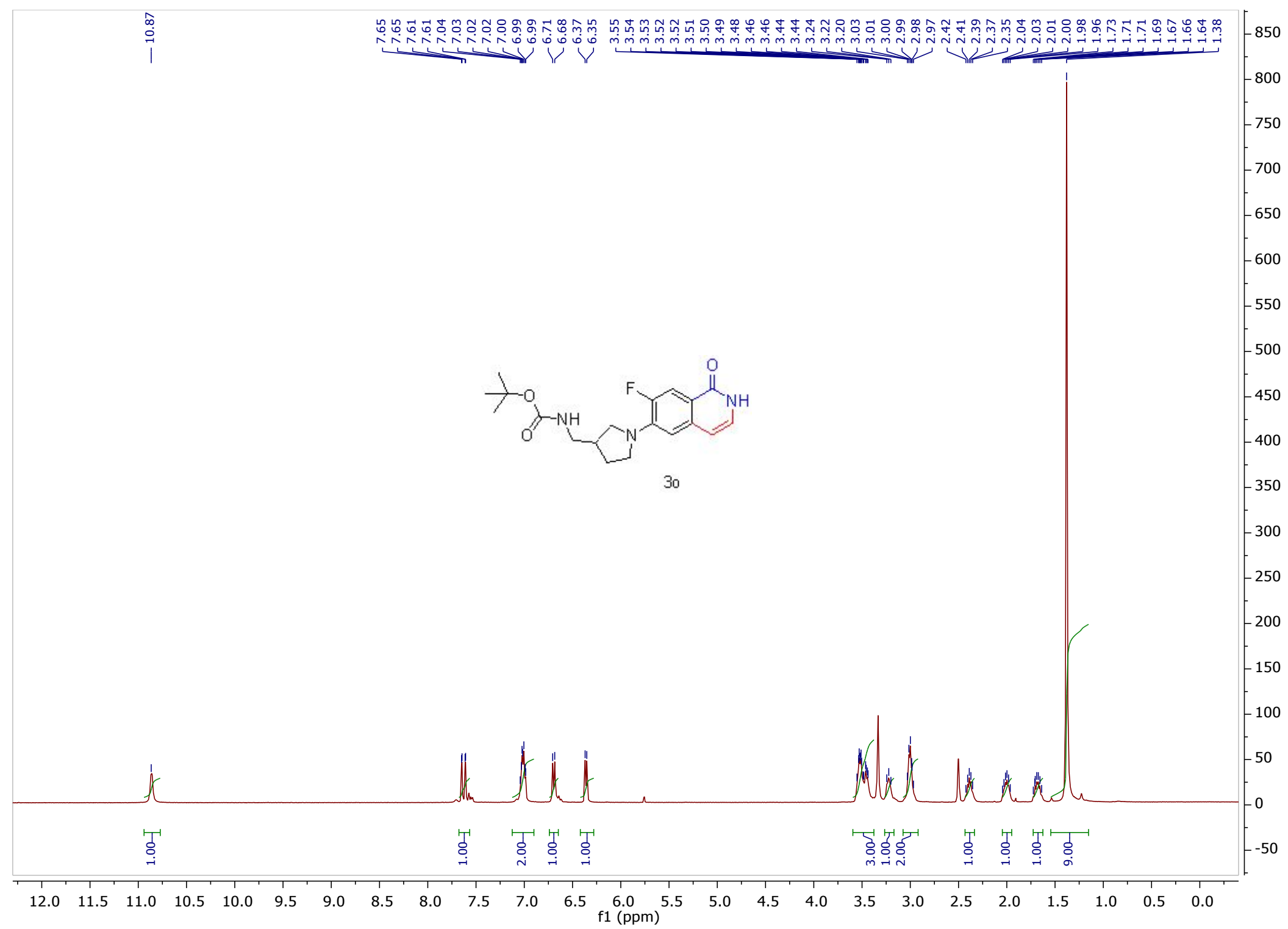


Figure S67. ${ }^{13} \mathrm{C}\left\{{ }^{1} \mathrm{H}\right\}-\mathrm{NMR}$ (101 MHz, DMSO-d6) for compound 30 .

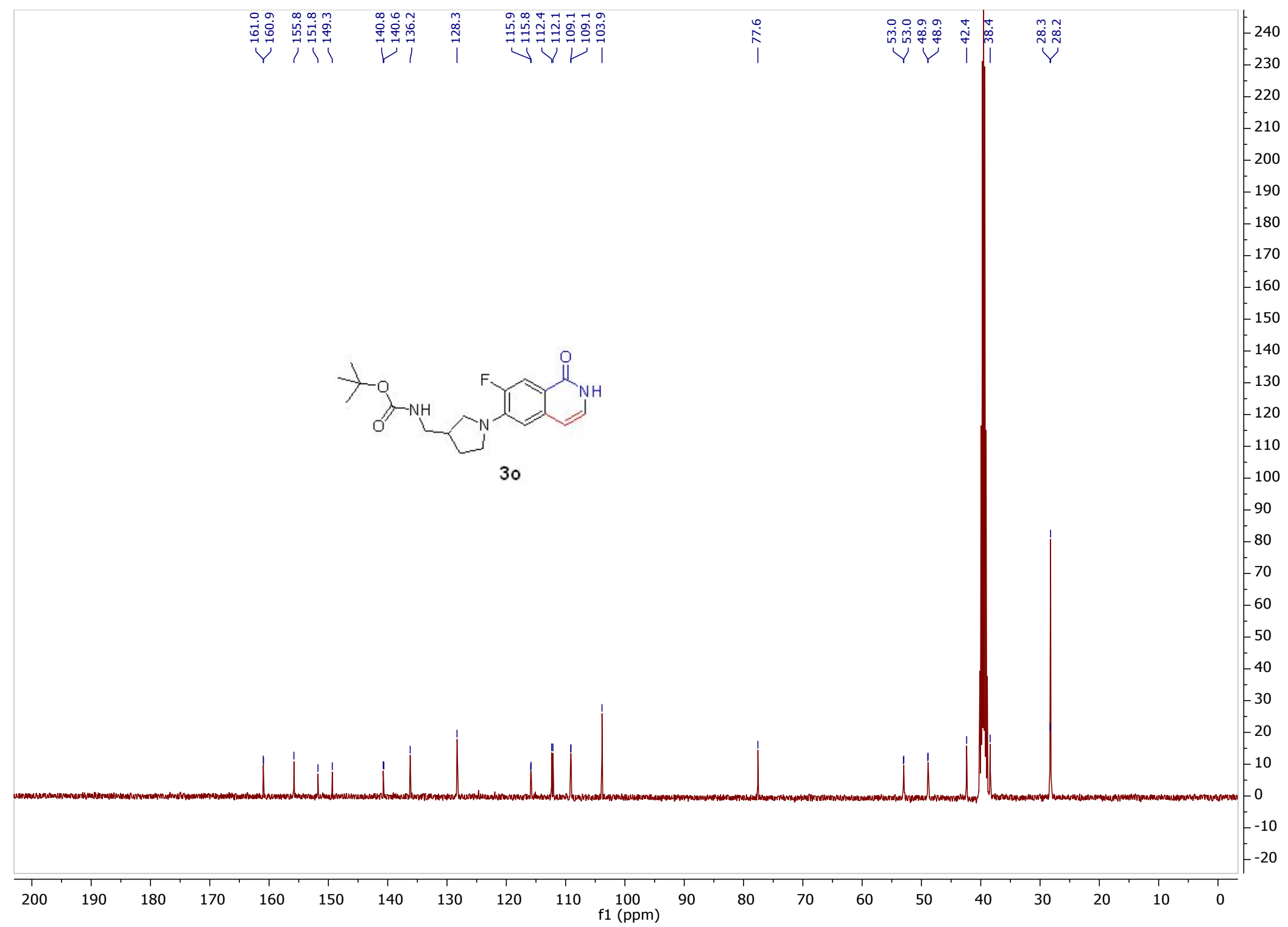


Figure S68. ${ }^{19} \mathrm{~F}-\mathrm{NMR}$ (376 MHz, DMSO-d6) for compound 30.

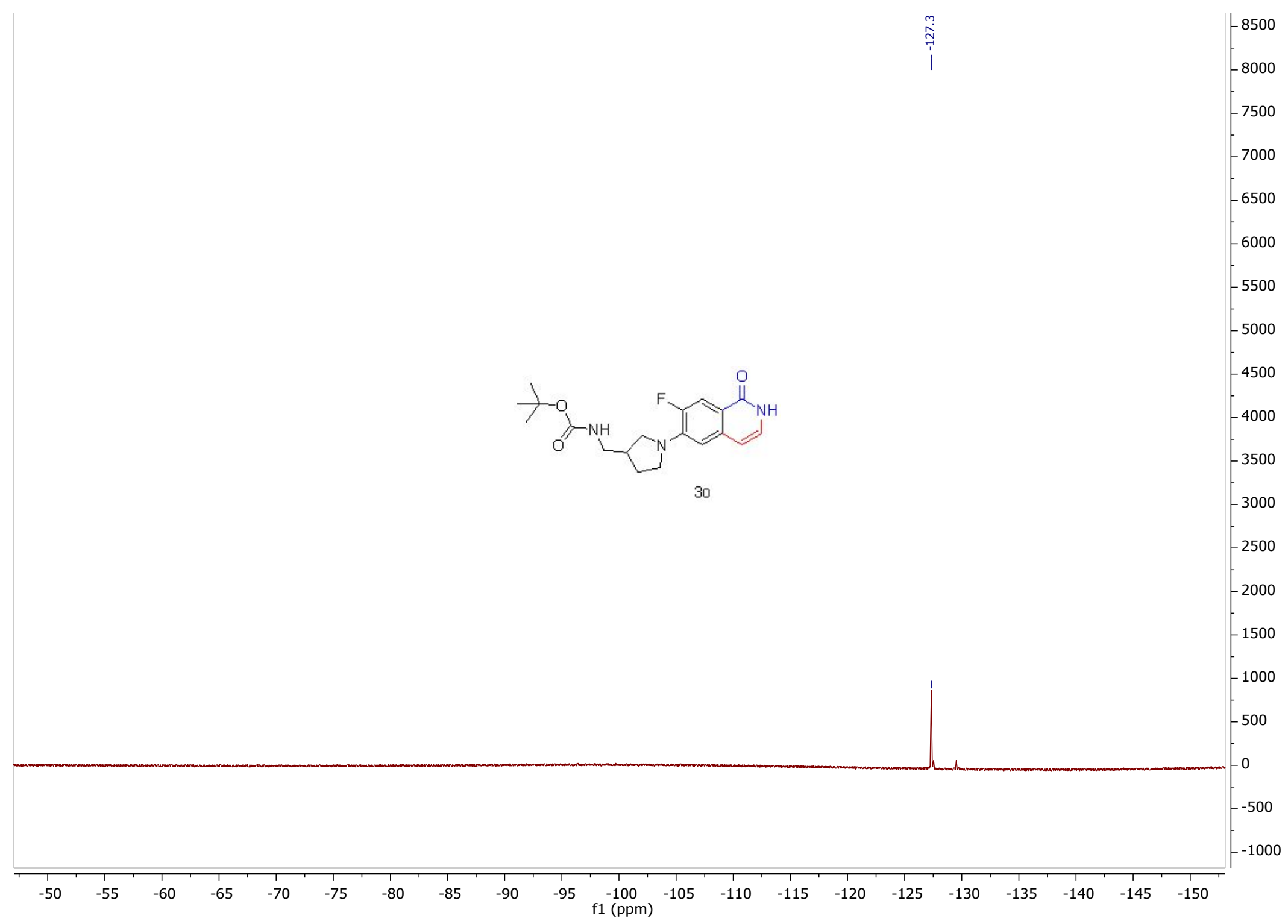


S3. NMR spectra for compounds 6a-6d.

Figure S69. ${ }^{1} \mathrm{H}-\mathrm{NMR}(400 \mathrm{MHz}$, DMSO-d6) for compound $6 \mathrm{a}$.

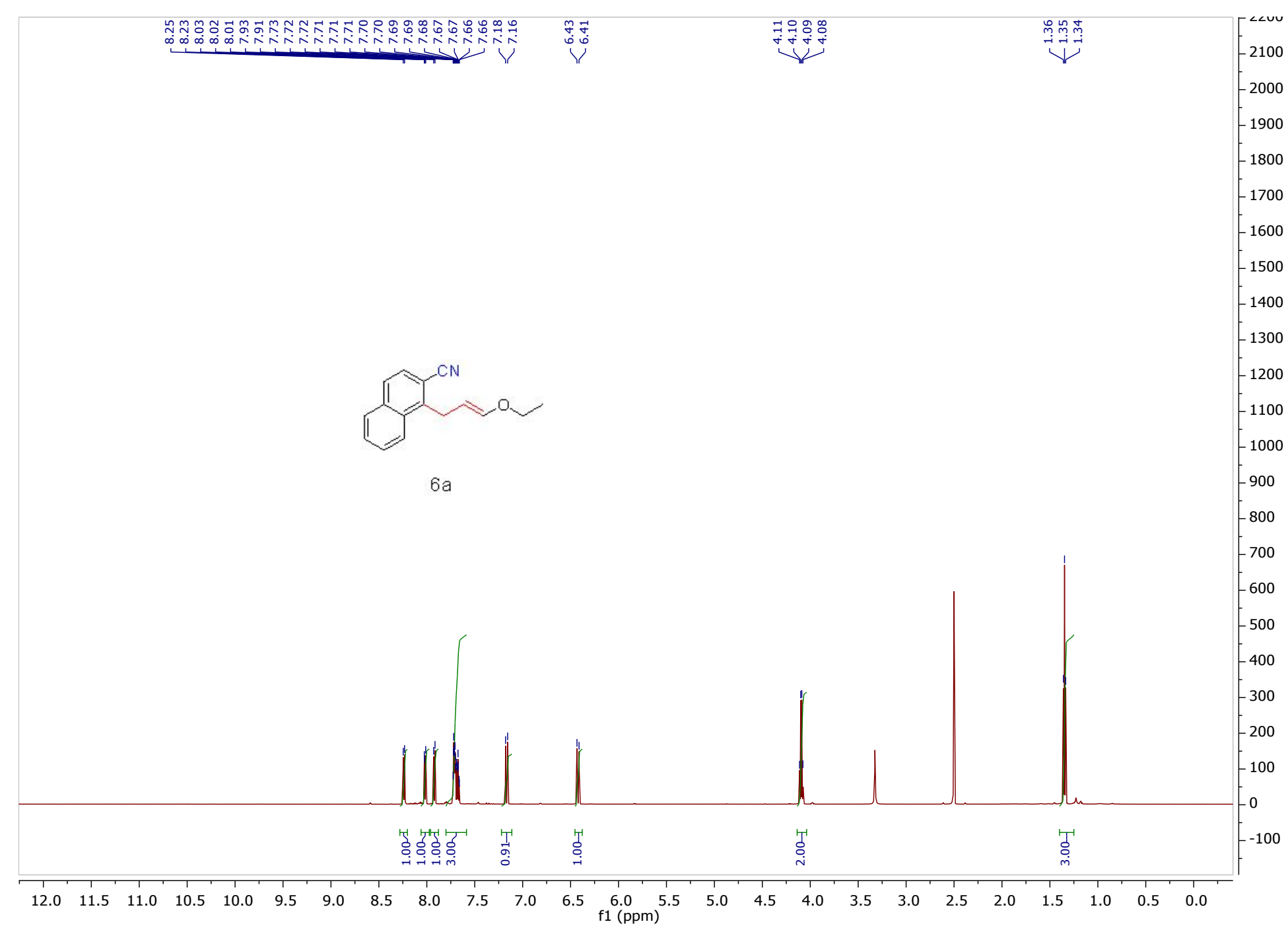


Figure S70. ${ }^{13} \mathrm{C}\left\{{ }^{1} \mathrm{H}\right\}-\mathrm{NMR}(151 \mathrm{MHz}$, DMSO-d6) for compound 6a.

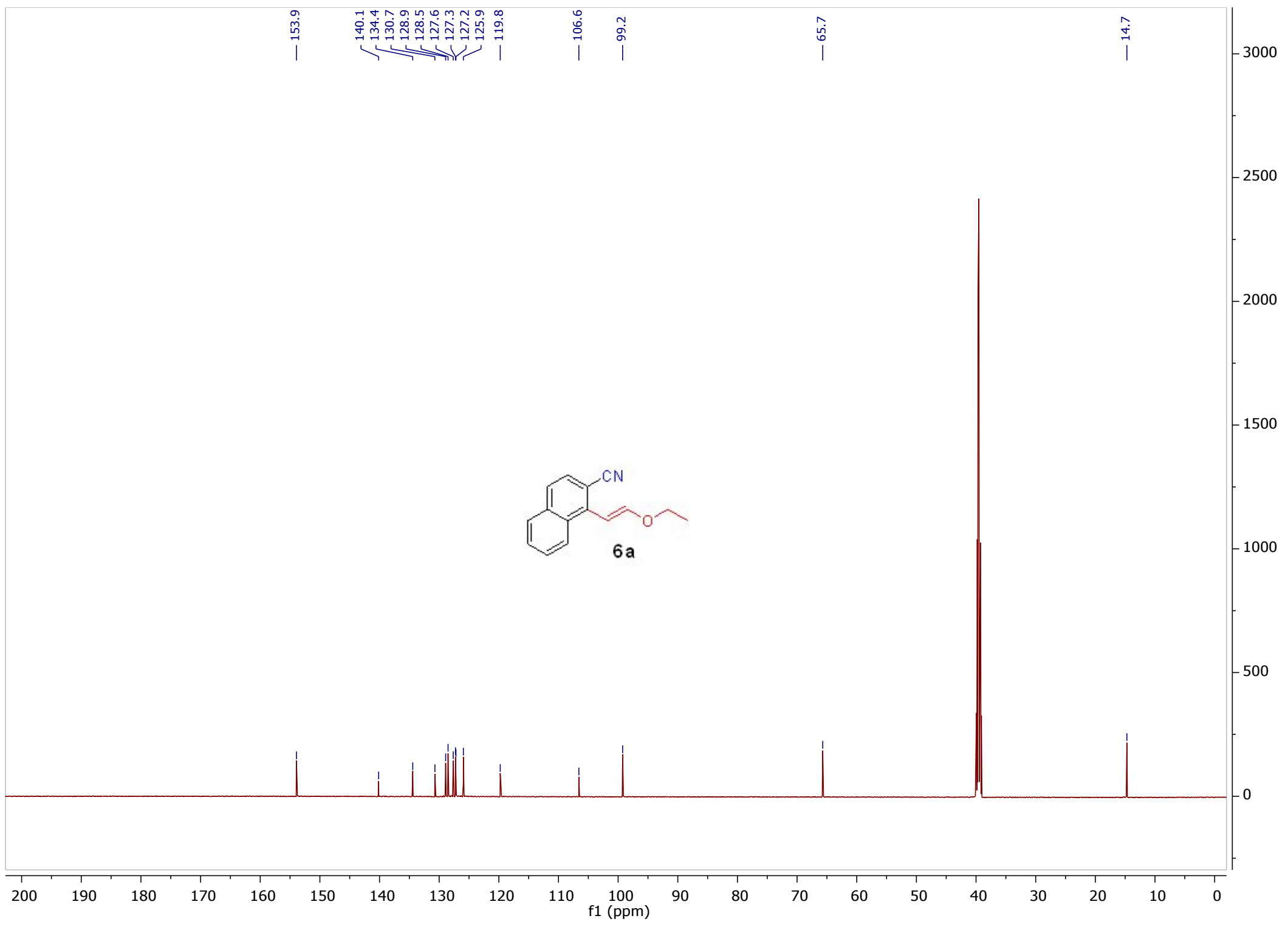

SI-71 
Figure S71. ${ }^{1} \mathrm{H}-\mathrm{NMR}(600 \mathrm{MHz}$, DMSO-d6) for compound $6 \mathrm{~b}$.

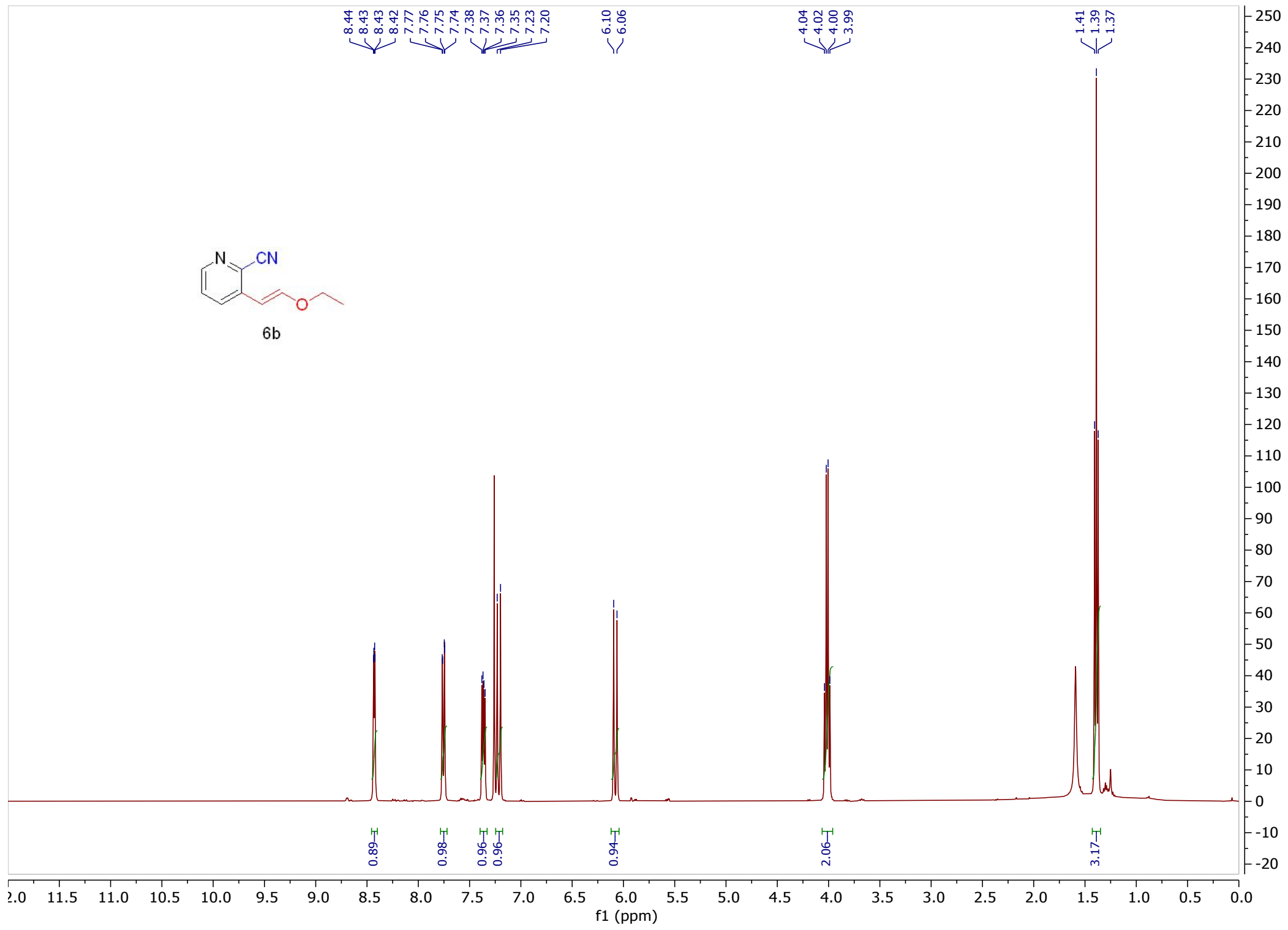

SI-72 
Figure S72. ${ }^{13} \mathrm{C}\left\{{ }^{1} \mathrm{H}\right\}-\mathrm{NMR}$ (151 MHz, DMSO-6d) for compound $6 \mathrm{~b}$.

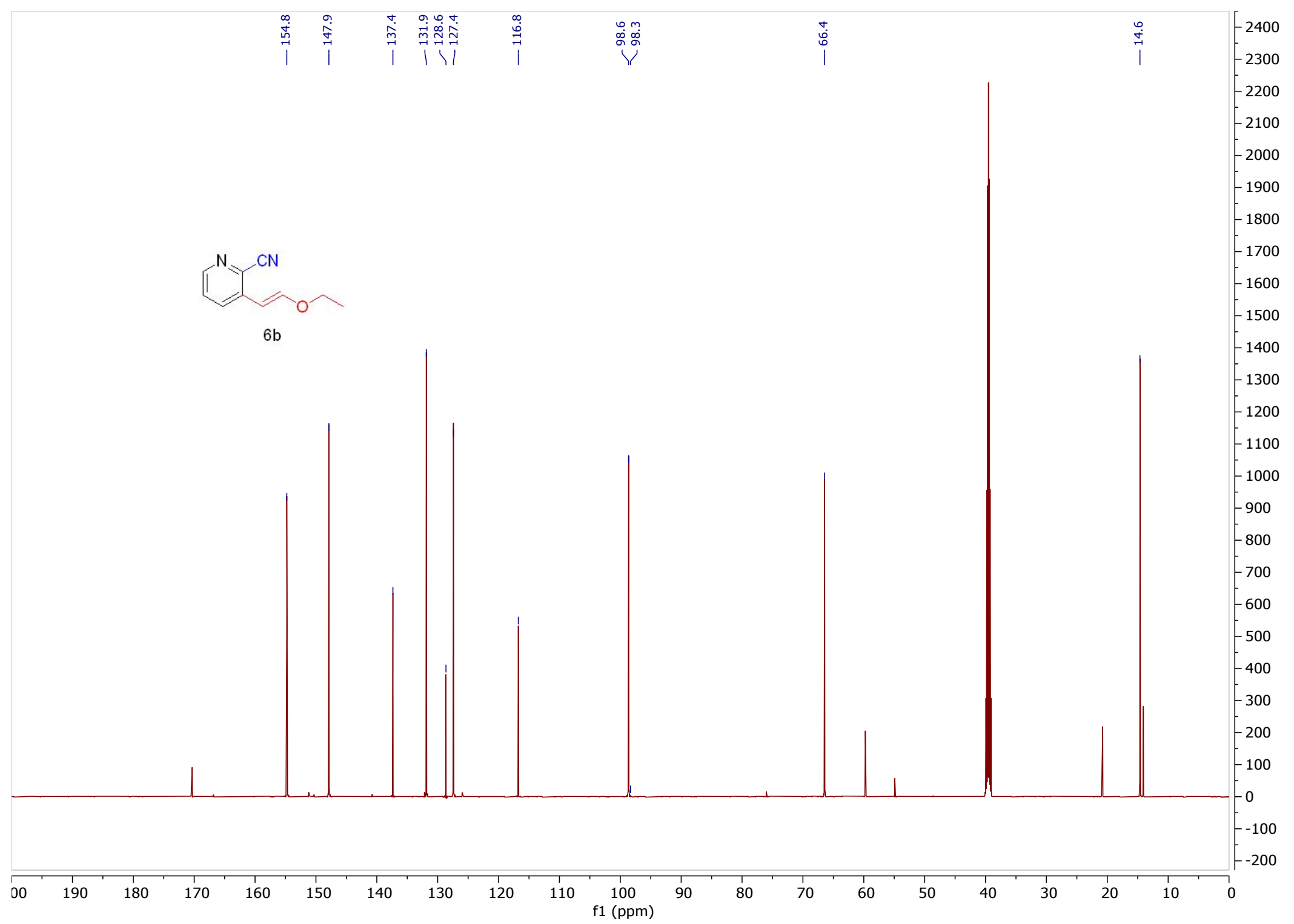


Figure S73. ${ }^{1} \mathrm{H}-\mathrm{NMR}$ (600 MHz, DMSO-d6) for compound $6 \mathrm{c}$.

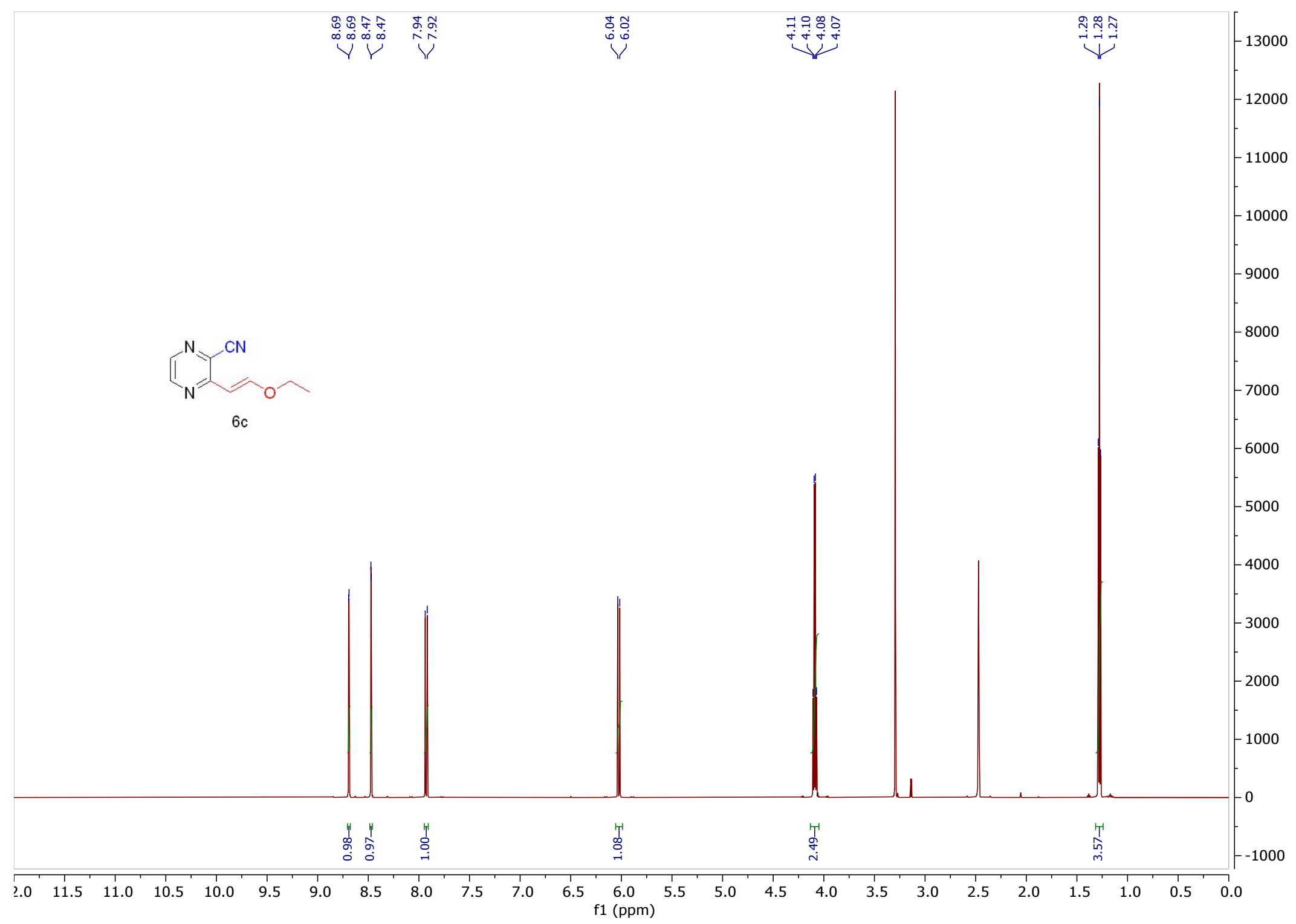


Figure S74. ${ }^{13} \mathrm{C}\left\{{ }^{1} \mathrm{H}\right\}-\mathrm{NMR}$ (151 MHz, DMSO-d6) for compound $6 \mathrm{c}$.

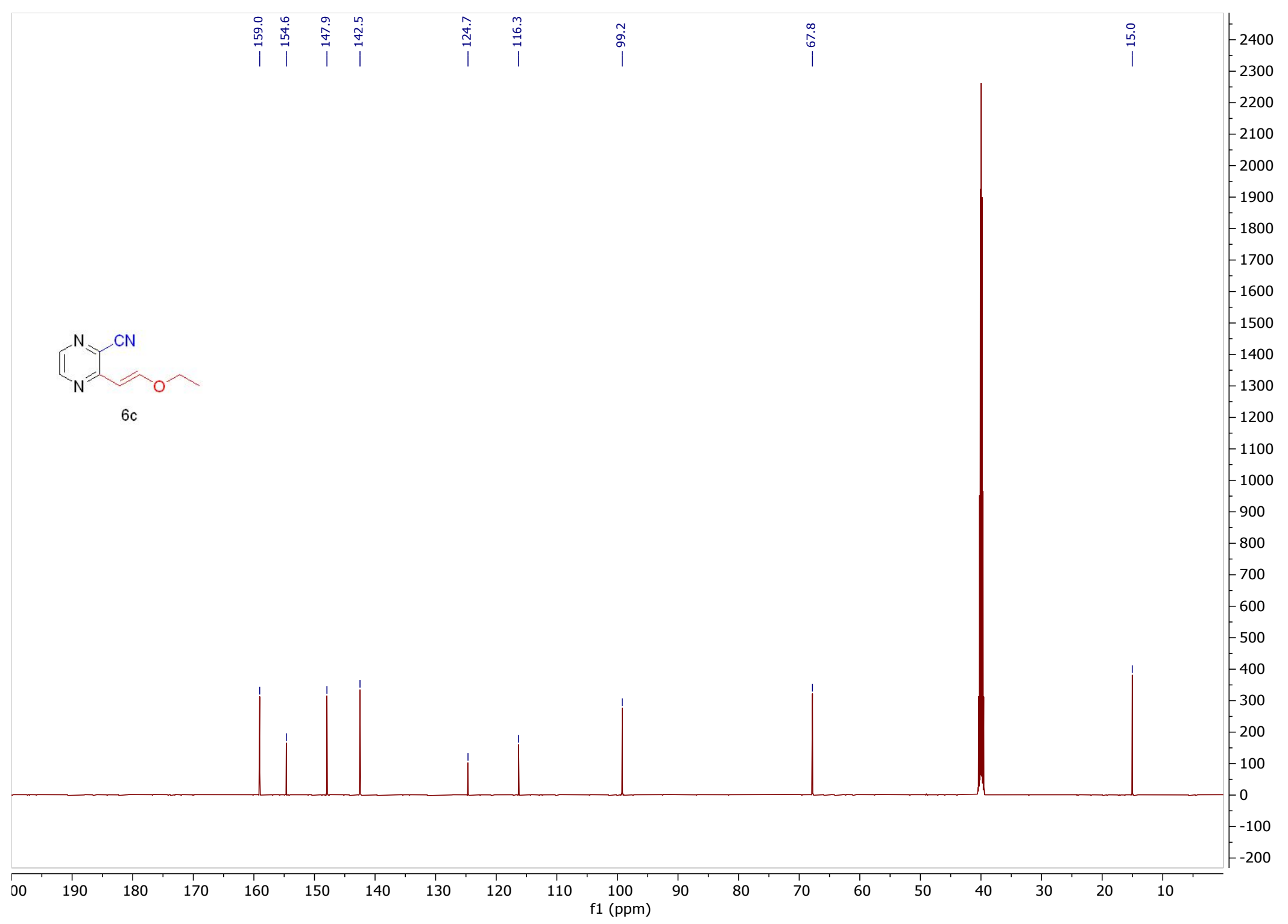


Figure S75. ${ }^{1} \mathrm{H}-\mathrm{NMR}\left(400 \mathrm{MHz}, \mathrm{CDCl}_{3}\right)$ for compound $6 \mathrm{~d}$.

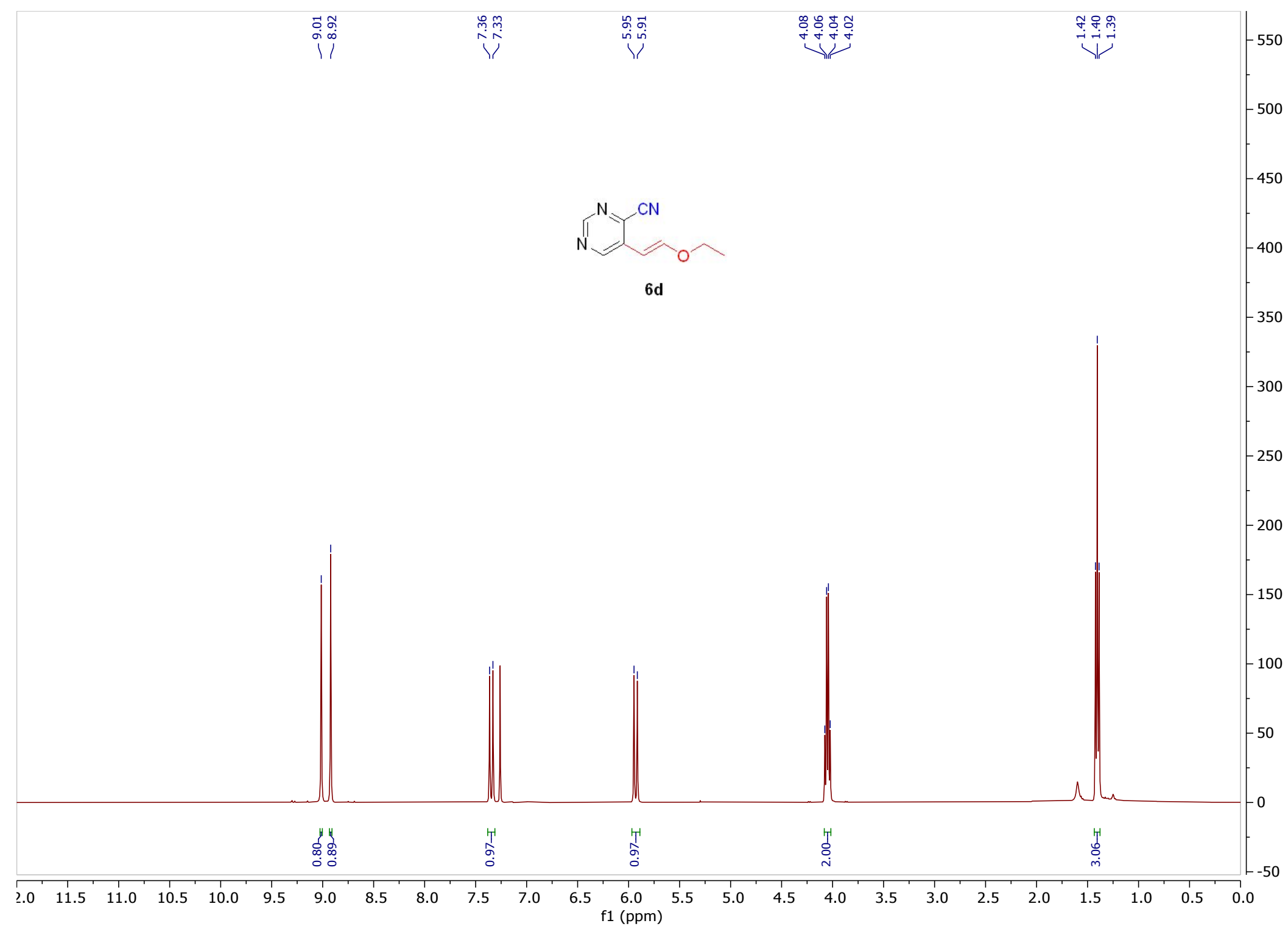


Figure S76. ${ }^{13} \mathrm{C}\left\{{ }^{1} \mathrm{H}\right\}-\mathrm{NMR}\left(101 \mathrm{MHz}, \mathrm{CDCl}_{3}\right)$ for compound $6 \mathrm{~d}$.

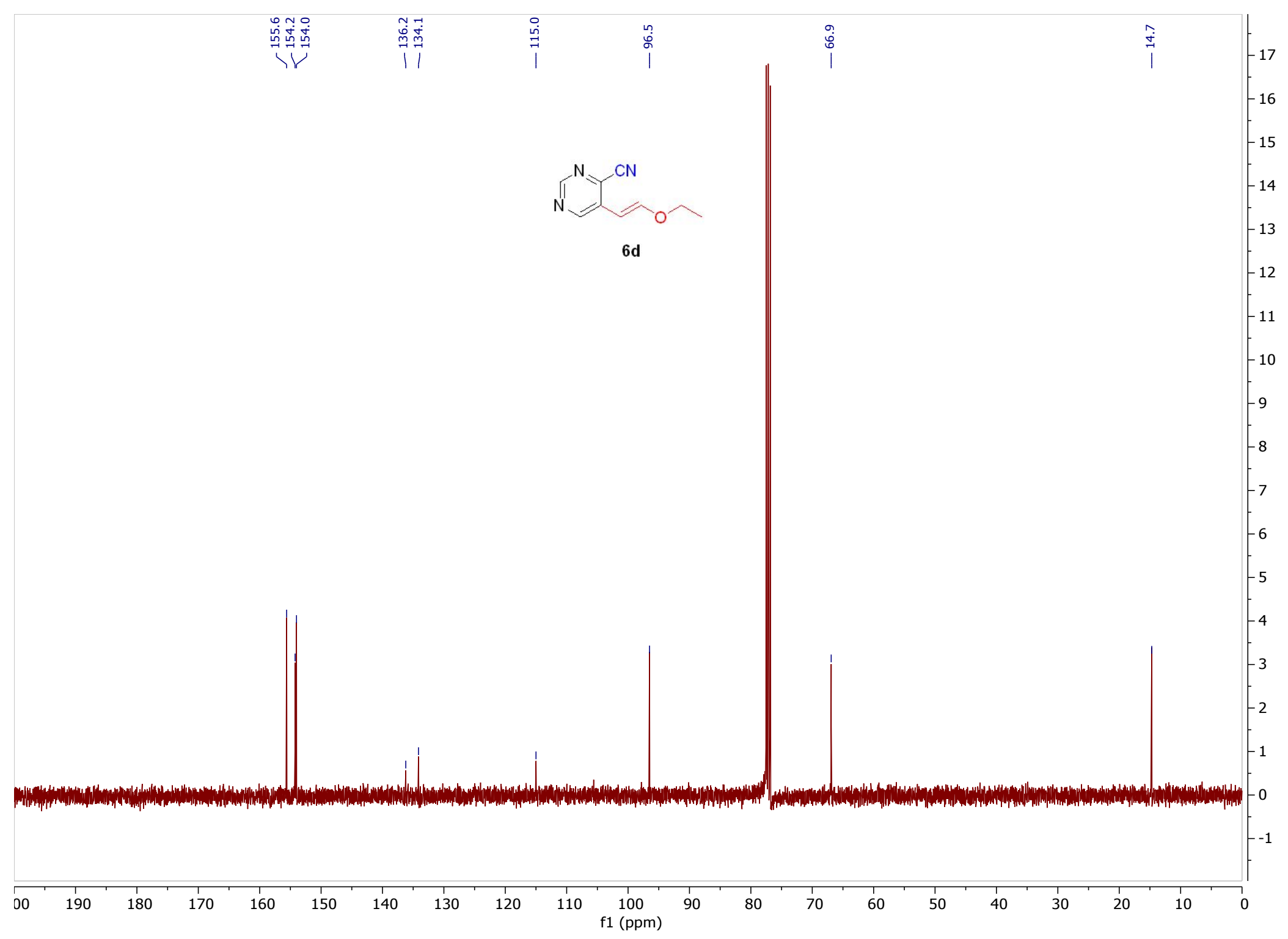


S4. NMR spectra for compounds 7a-7d.

Figure S77. ${ }^{1} \mathrm{H}-\mathrm{NMR}(500 \mathrm{MHz}$, DMSO-d6) for compound 7a.

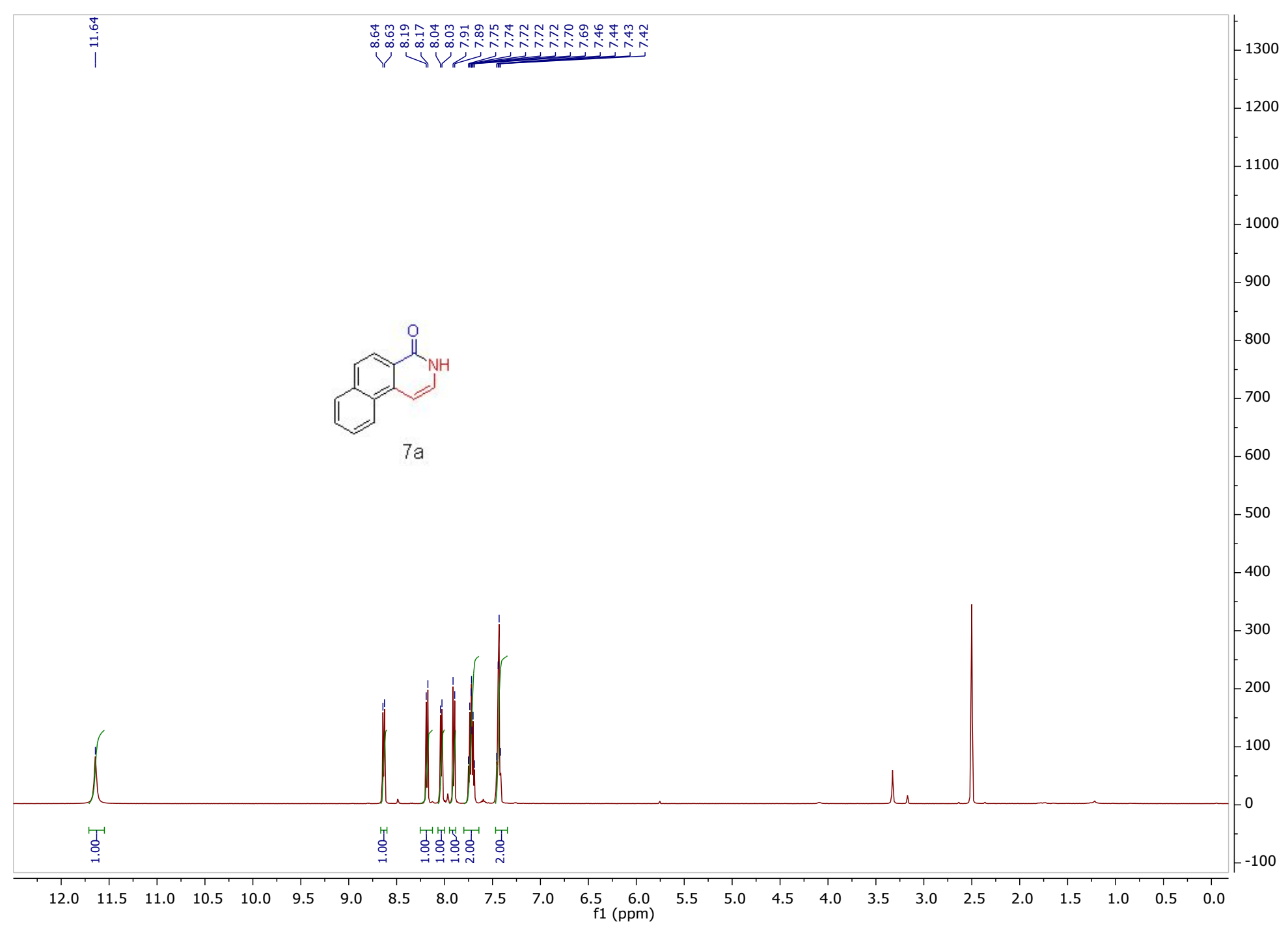


Figure S78. ${ }^{13} \mathrm{C}\left\{{ }^{1} \mathrm{H}\right\}-\mathrm{NMR}$ (126 MHz, DMSO-d6) for compound 7a.

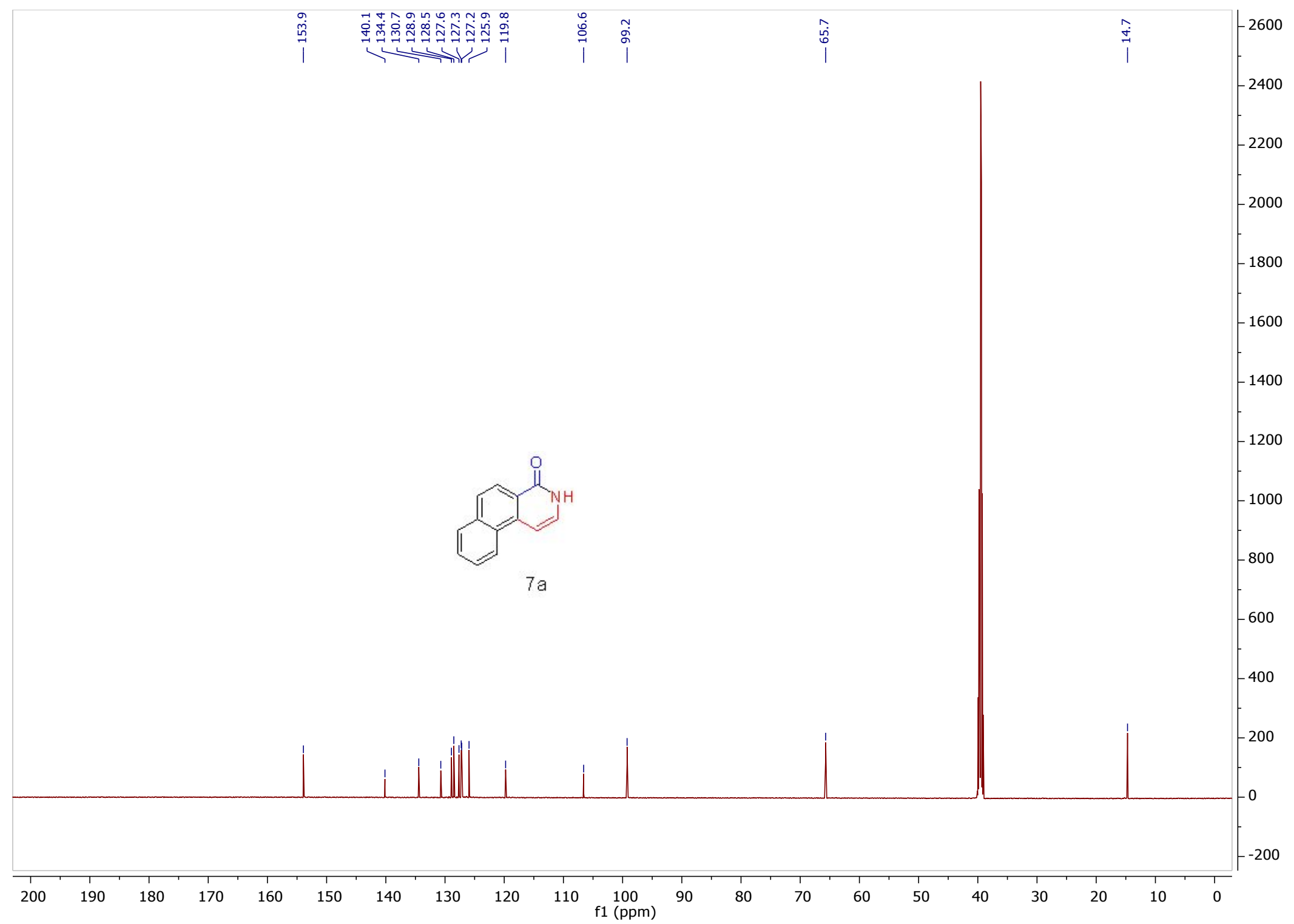


Figure S79. ${ }^{1} \mathrm{H}-\mathrm{NMR}$ (400 MHz, DMSO-d6) for compound 7b.

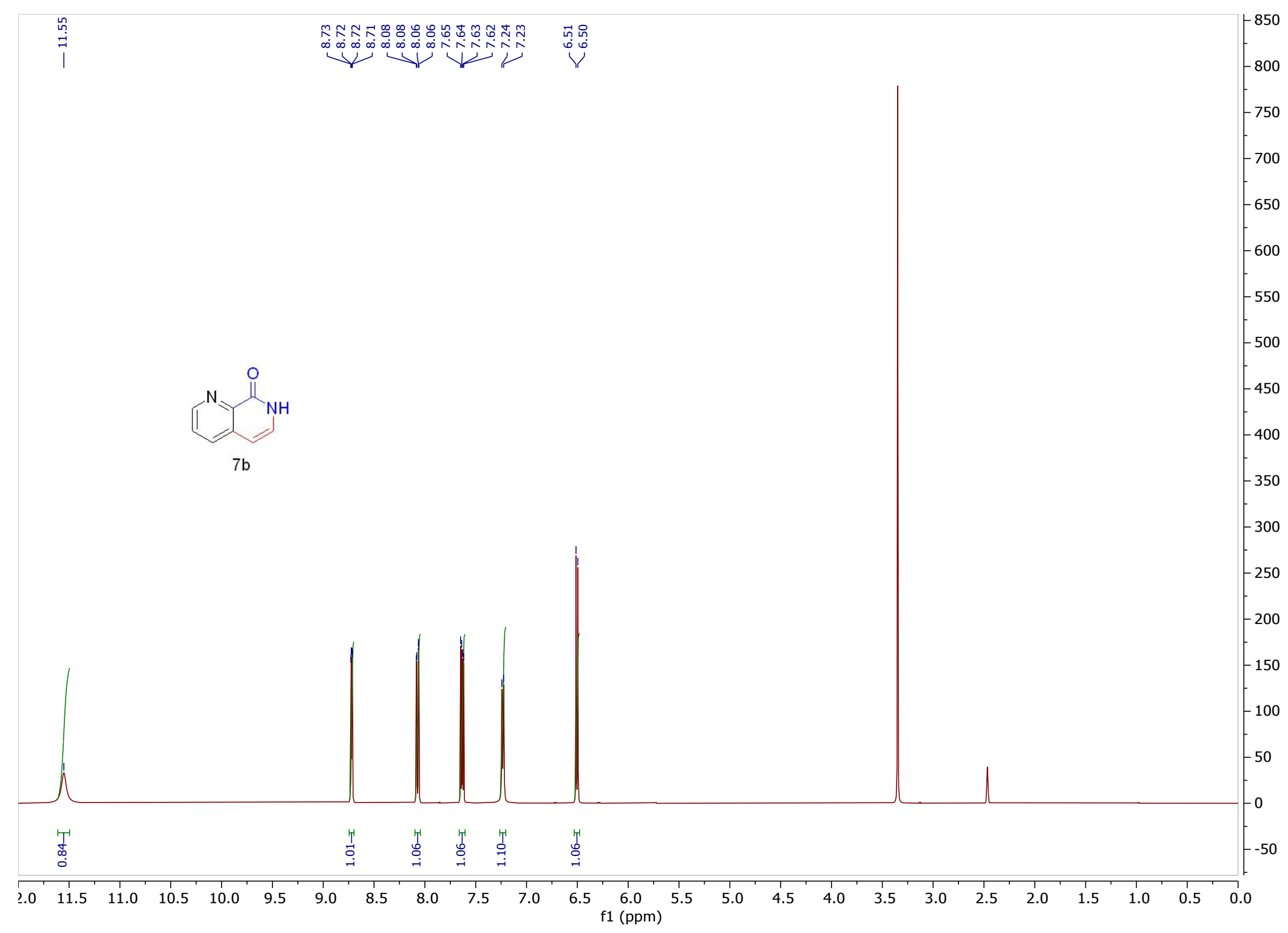


Figure $580 .{ }^{13} \mathrm{C}\left\{{ }^{1} \mathrm{H}\right\}-\mathrm{NMR}$ for (101 MHz, DMSO-d6) compound 7b.

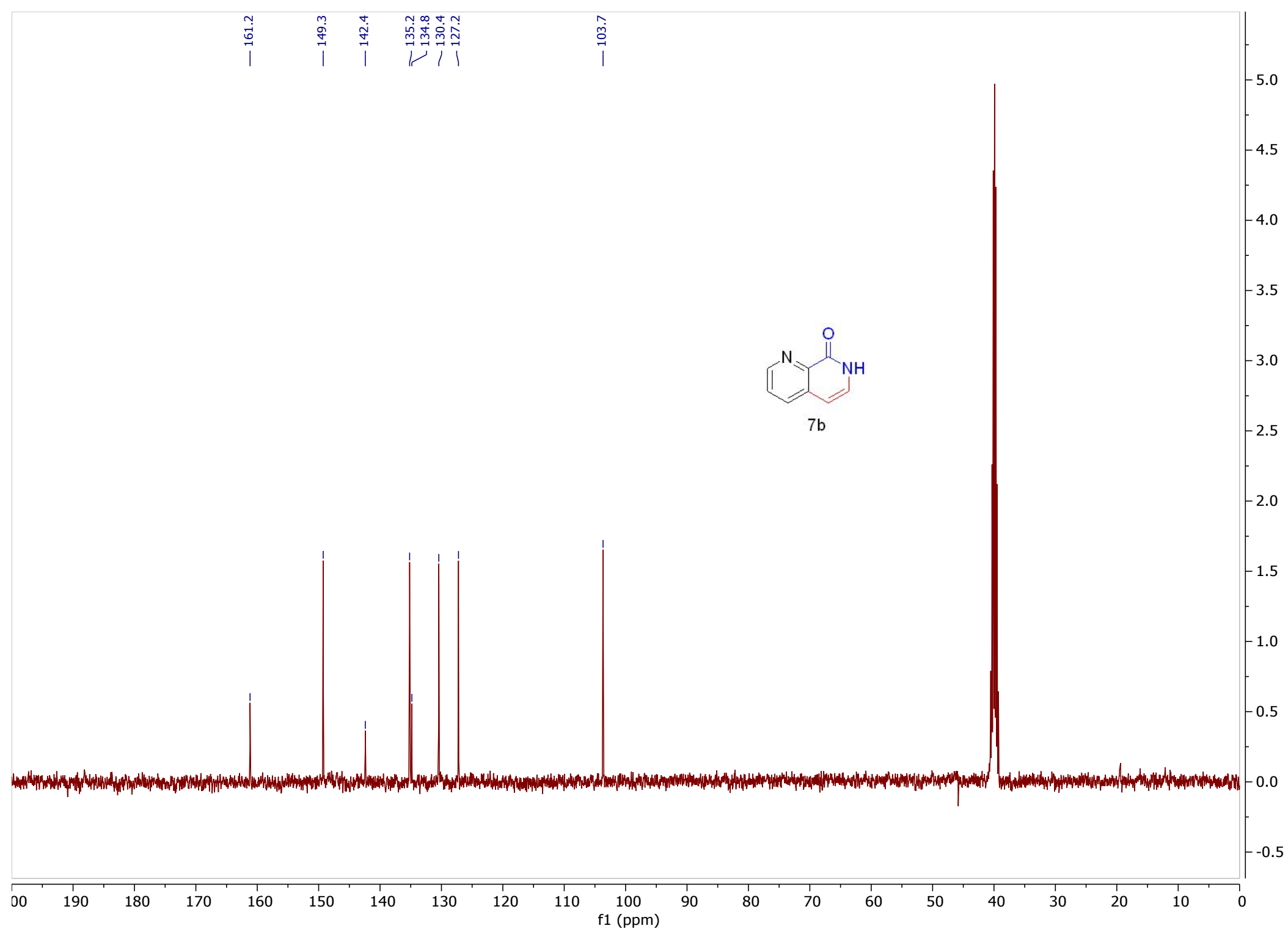


Figure S81. ${ }^{1} \mathrm{H}-\mathrm{NMR}(400 \mathrm{MHz}$, DMSO-d6) for compound 7c.

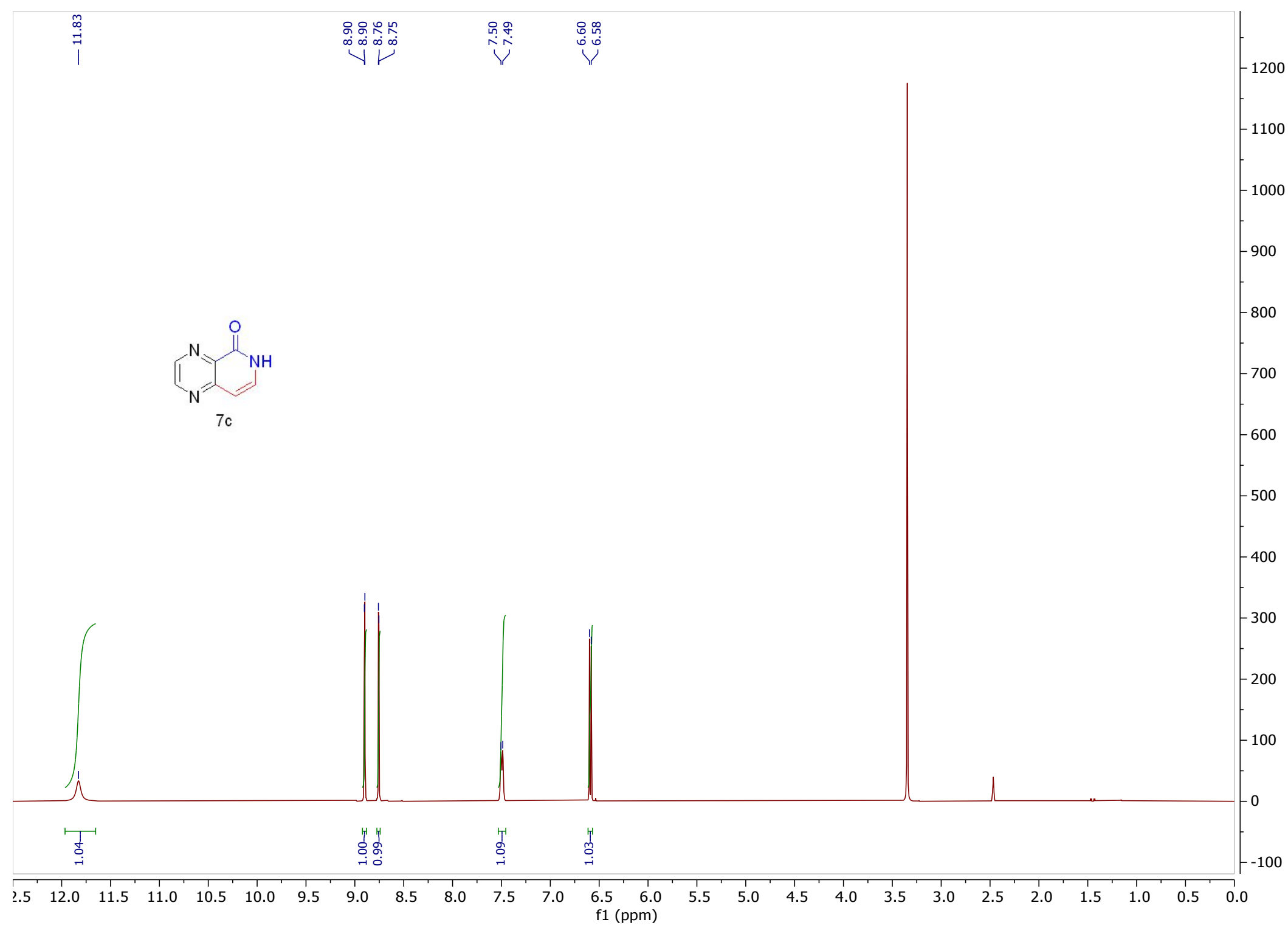


Figure S82. ${ }^{13} \mathrm{C}\left\{{ }^{1} \mathrm{H}\right\}-\mathrm{NMR}$ (101 MHz, DMSO-d6) for compound 7c.

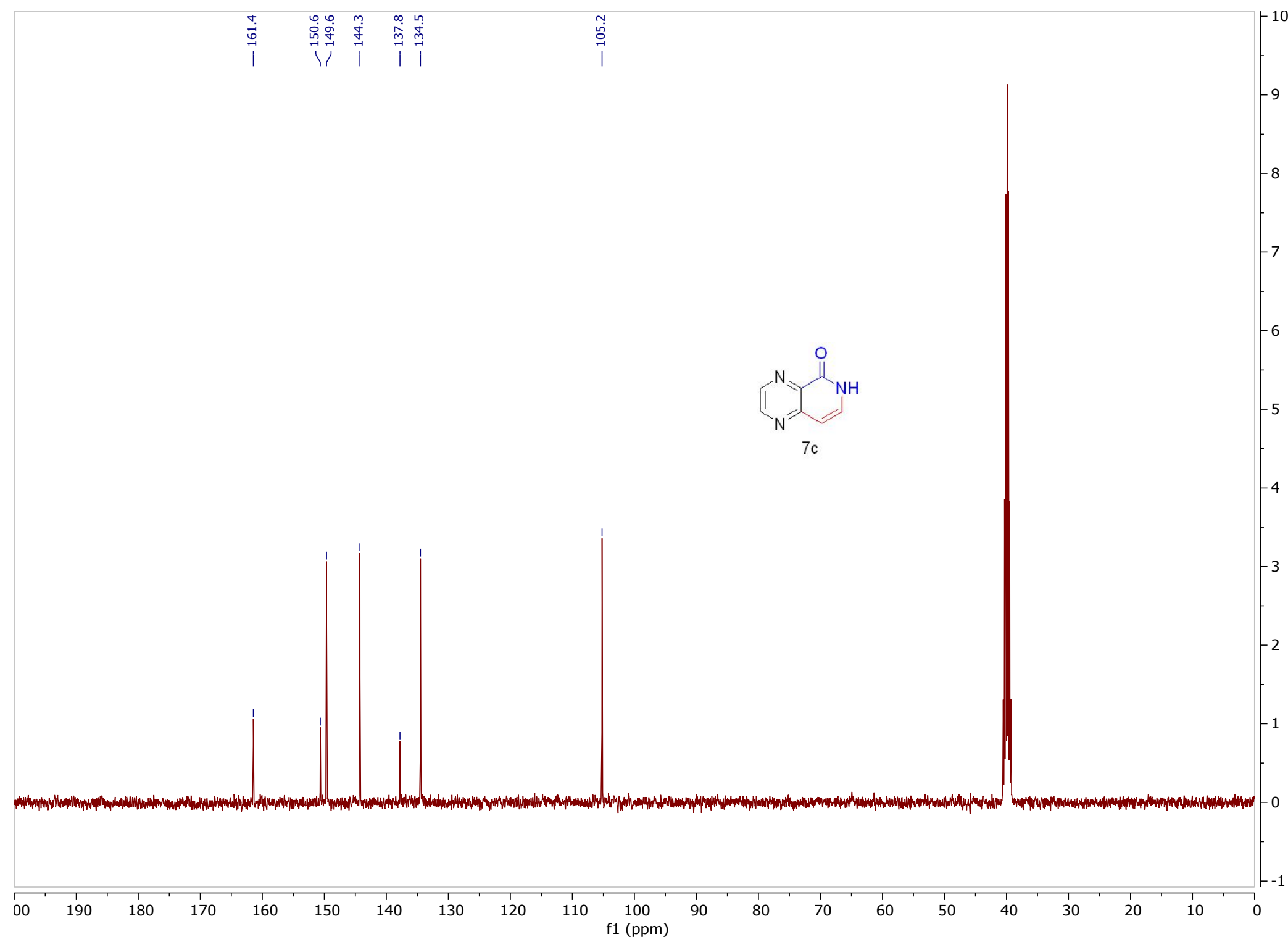


Figure S83. ${ }^{1} \mathrm{H}-\mathrm{NMR}$ (400 MHz, DMSO-d6) for compound 7d.

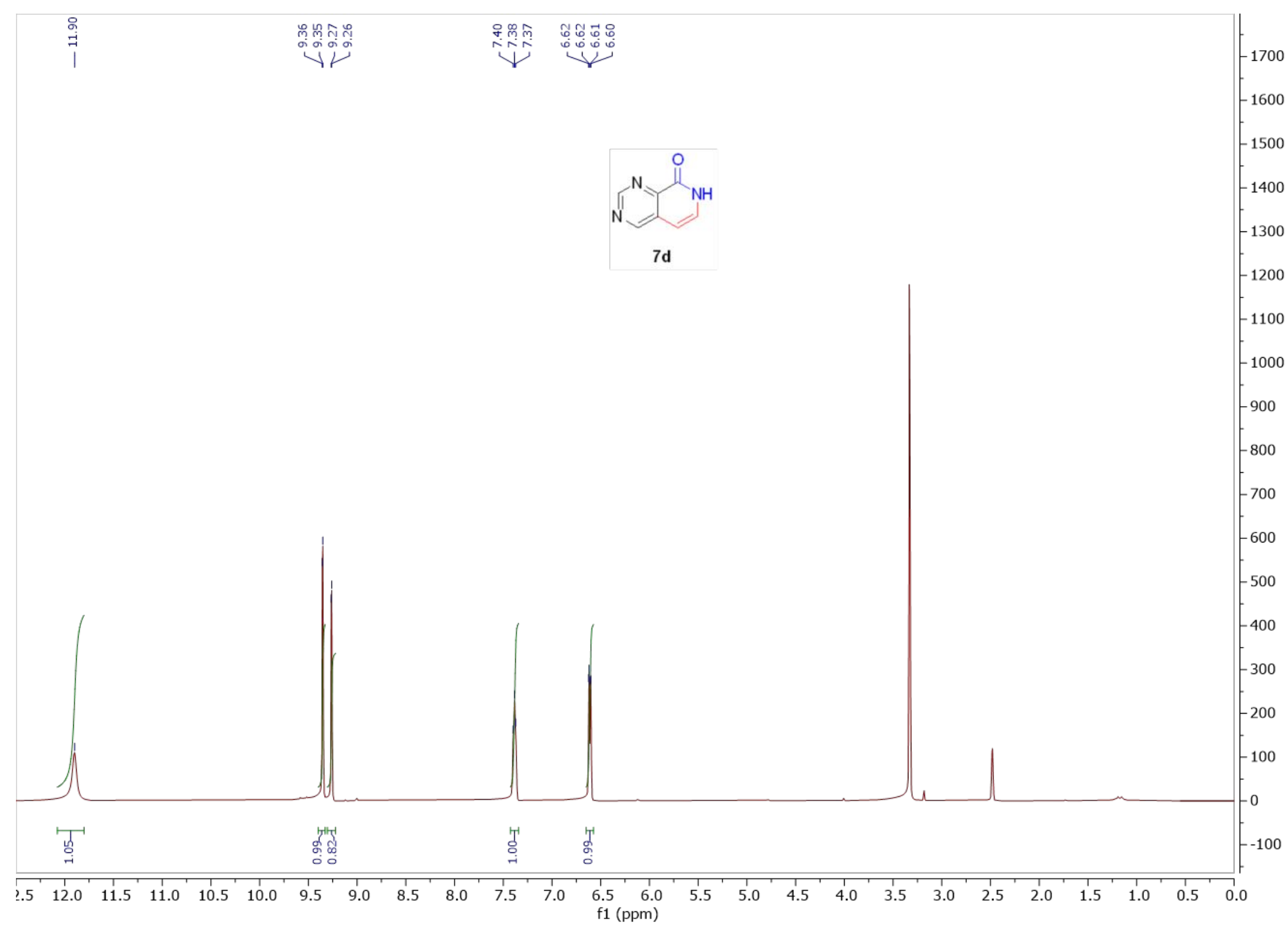


Figure S84. ${ }^{13} \mathrm{C}\left\{{ }^{1} \mathrm{H}\right\}-\mathrm{NMR}$ (101 MHz, DMSO-d6) for compound 7d.

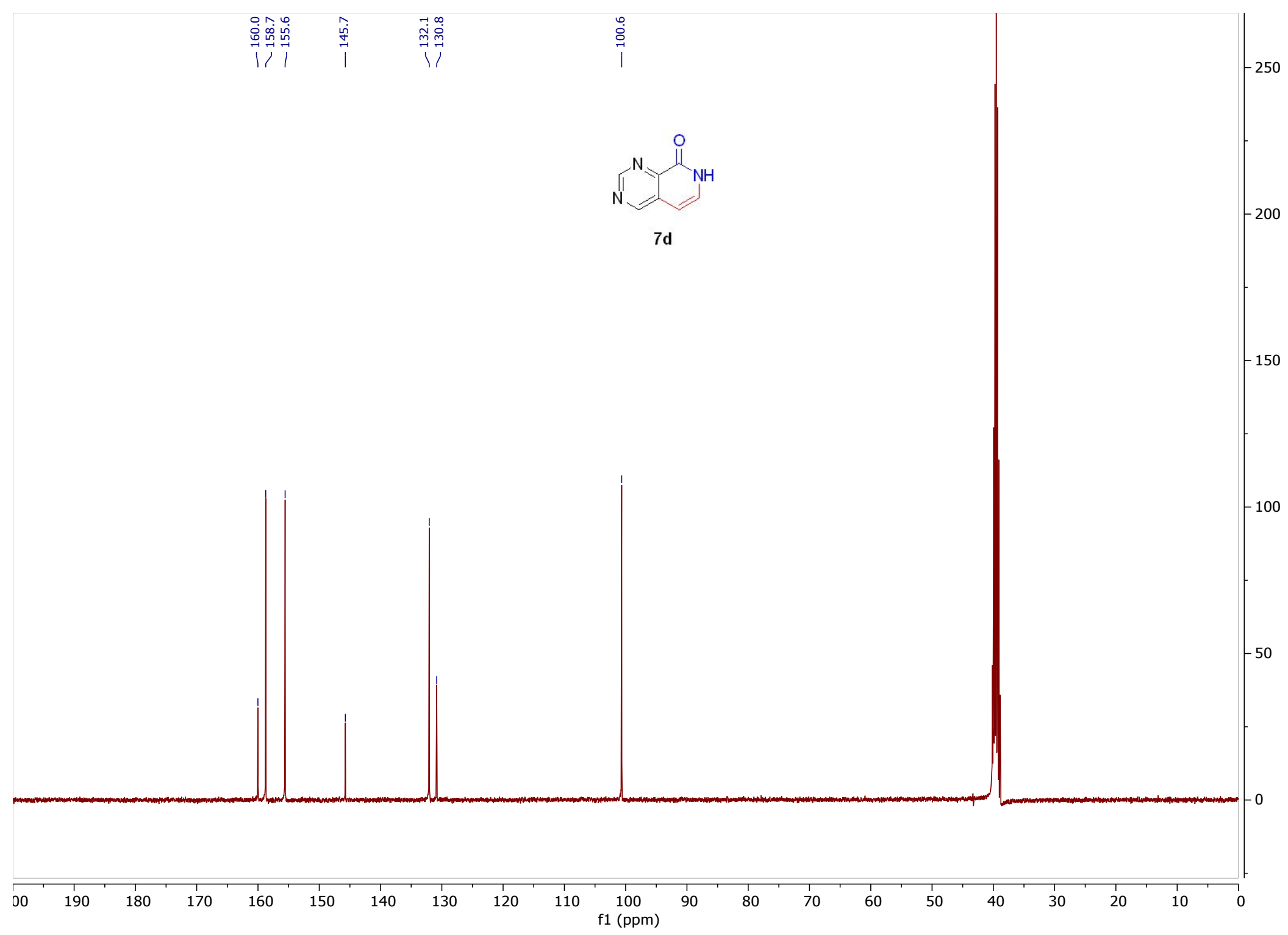


S5. NMR spectra for compounds 10 and 7d'.

Figure $585 .{ }^{13} \mathrm{H}-\mathrm{NMR}\left(400 \mathrm{MHz}, \mathrm{CDCl}_{3}\right)$ for compound 10

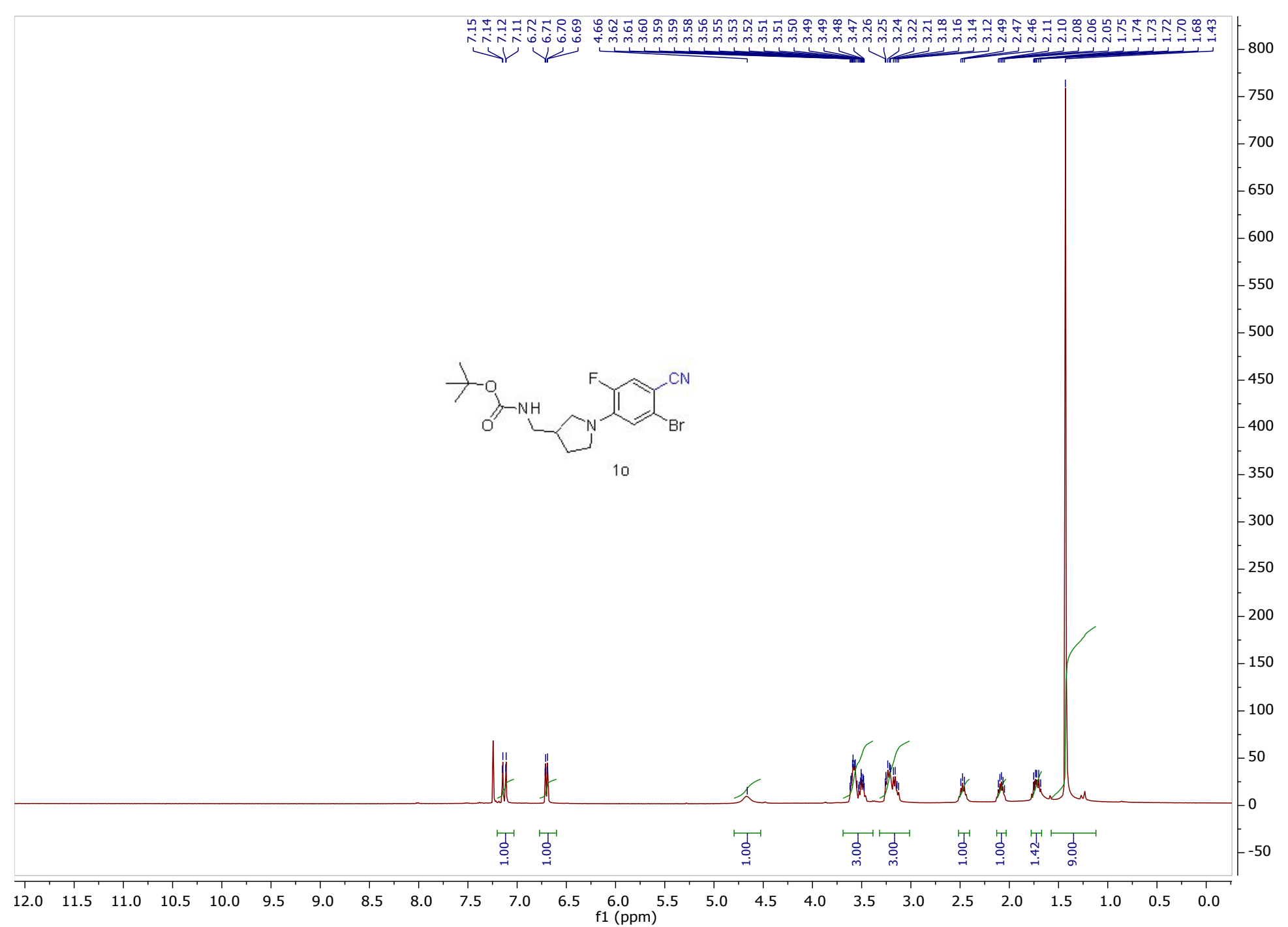


Figure S86. ${ }^{13} \mathrm{C}\left\{{ }^{1} \mathrm{H}\right\}-\mathrm{NMR}\left(101 \mathrm{MHz}, \mathrm{CDCl}_{3}\right)$ for compound 10

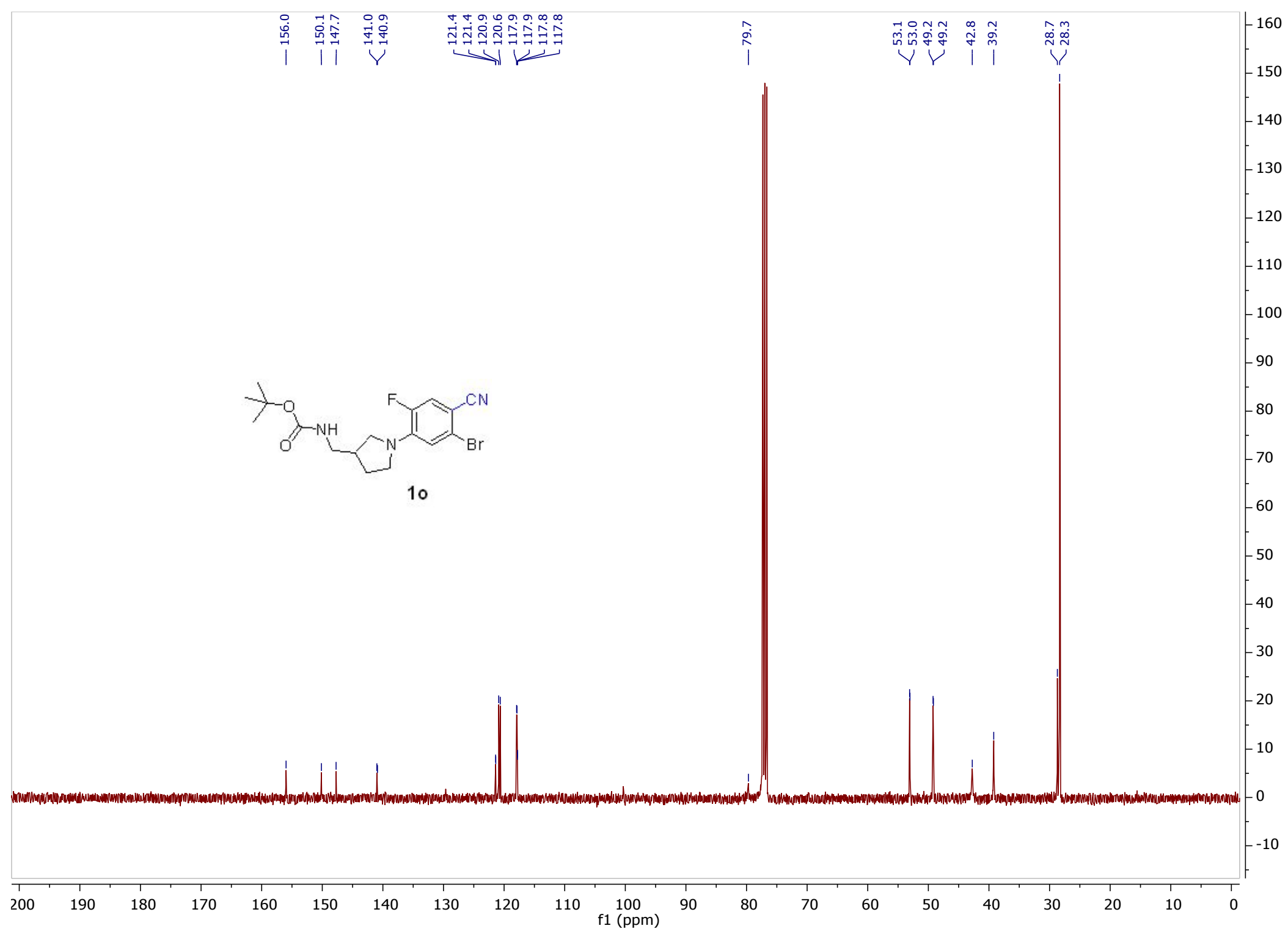


Figure S87. ${ }^{19} \mathrm{~F}-\mathrm{NMR}\left(376 \mathrm{MHz}, \mathrm{CDCl}_{3}\right.$ ) for compound 10

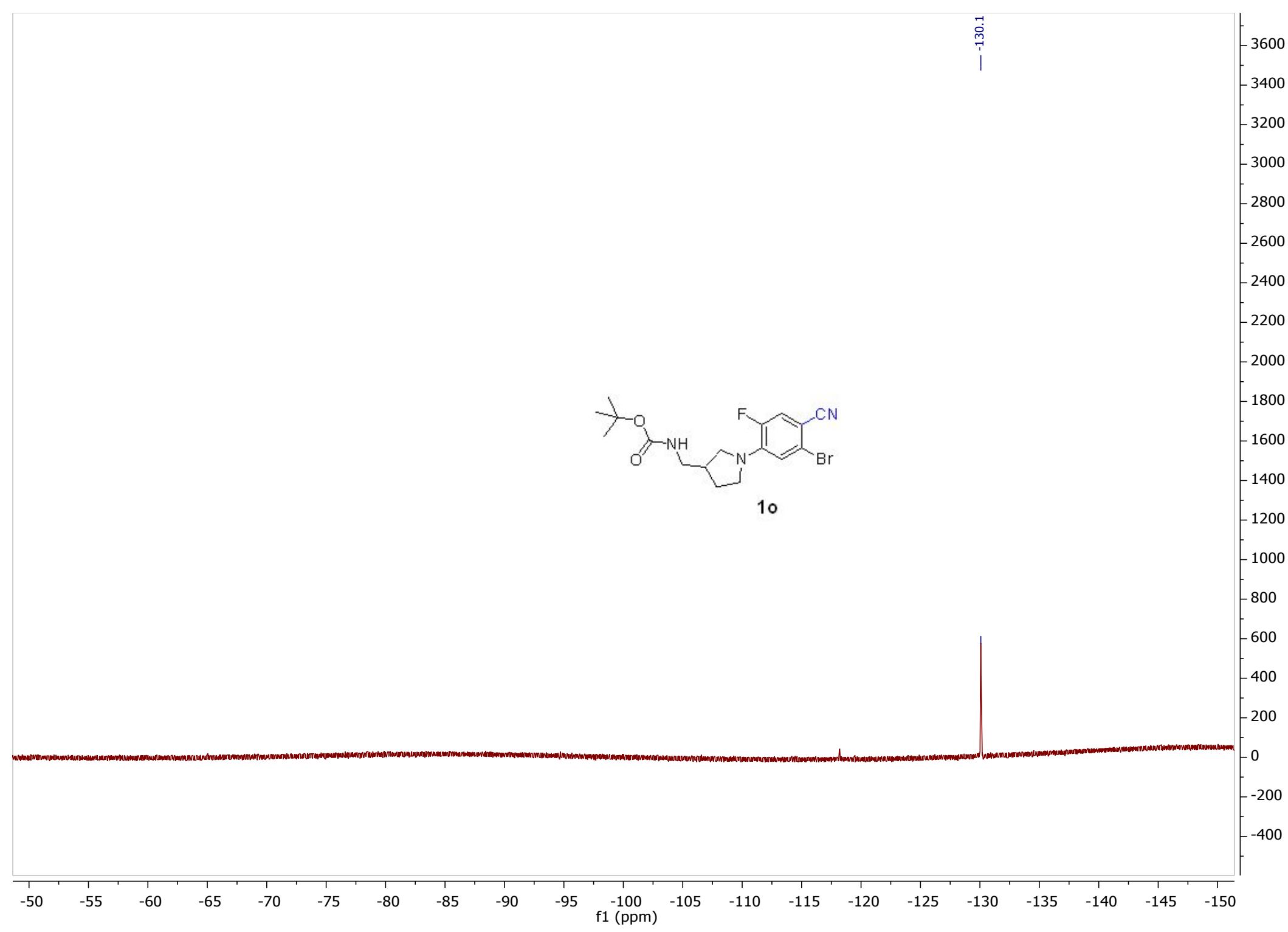


Figure S88. ${ }^{1} \mathrm{H}-\mathrm{NMR}$ (400 MHz, $\mathrm{CDCl}_{3}$ ) for compound 7d' (amide intermediate).

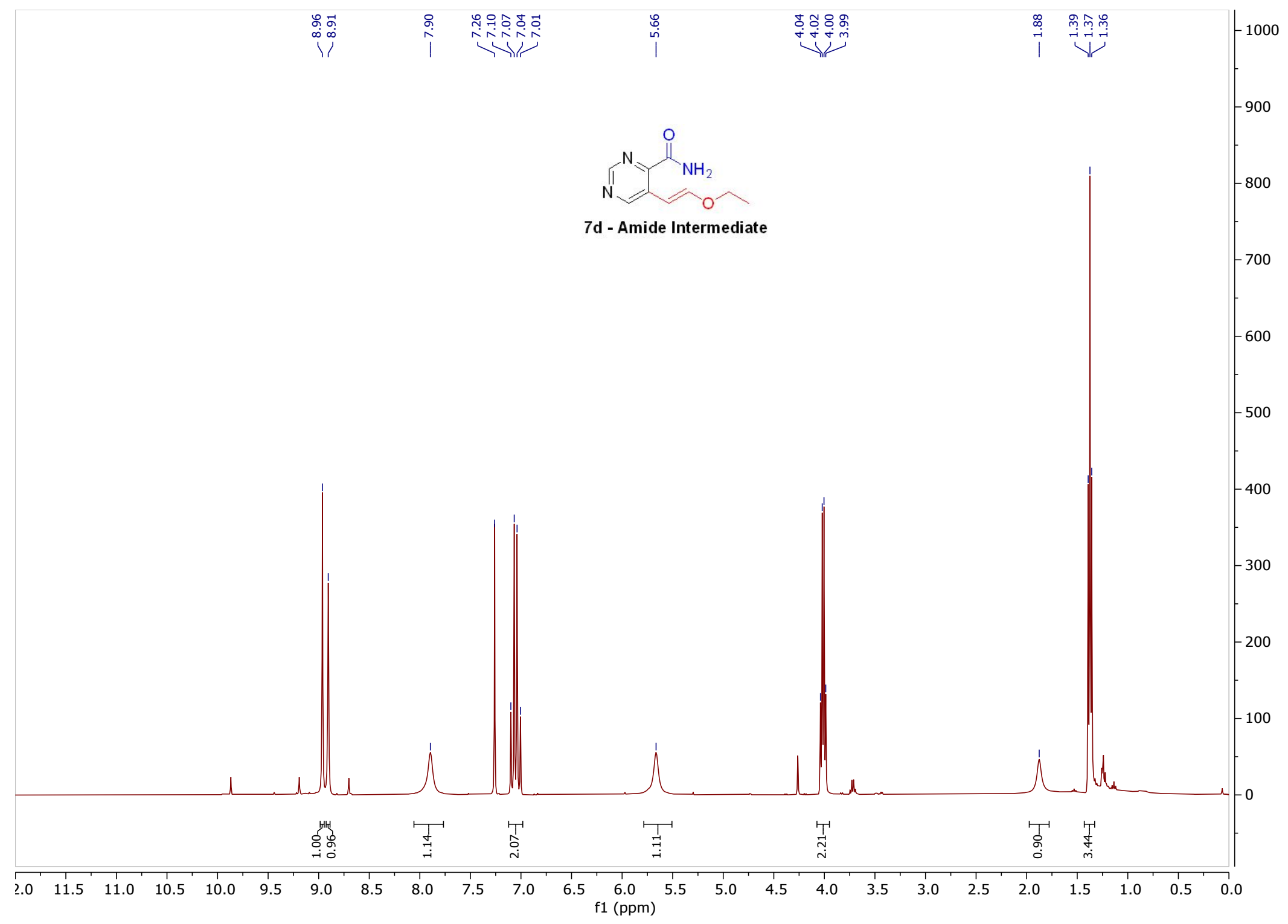

